\title{
WestVirginiaUniversity
}

THE RESEARCH REPOSITORY @ WVU

Graduate Theses, Dissertations, and Problem Reports

2009

\section{Random KNN modeling and variable selection for high dimensional data}

Shengqiao Li

West Virginia University

Follow this and additional works at: https://researchrepository.wvu.edu/etd

\section{Recommended Citation}

$\mathrm{Li}$, Shengqiao, "Random KNN modeling and variable selection for high dimensional data" (2009). Graduate Theses, Dissertations, and Problem Reports. 4492.

https://researchrepository.wvu.edu/etd/4492

This Dissertation is protected by copyright and/or related rights. It has been brought to you by the The Research Repository @ WVU with permission from the rights-holder(s). You are free to use this Dissertation in any way that is permitted by the copyright and related rights legislation that applies to your use. For other uses you must obtain permission from the rights-holder(s) directly, unless additional rights are indicated by a Creative Commons license in the record and/ or on the work itself. This Dissertation has been accepted for inclusion in WVU Graduate Theses, Dissertations, and Problem Reports collection by an authorized administrator of The Research Repository @ WVU.

For more information, please contact researchrepository@mail.wvu.edu. 


\title{
Random KNN Modeling and Variable Selection for High Dimensional Data
}

\author{
Shengqiao Li
}

\author{
Dissertation submitted to the \\ College of Engineering and Mineral Resources \\ at West Virginia University \\ in partial fulfillment of the requirements \\ for the degree of
}
Doctor of Philosophy
in
Computer Science

\author{
Graduate Committee: \\ Associate Professor Donald A. Adjeroh, Chair \\ Professor E. James Harner, Chair \\ Associate Professor Elaine M. Eschen \\ Associate Professor Arun Ross \\ Associate Professor Robert Mnatsakanov
}

Lane Department of Computer Science \& Electrical Engineering

Morgantown, West Virginia
2009

Keywords: Classification, Regression, Feature Selection, Bioinformatics Copyright 2009 Shengqiao Li 


\section{ABSTRACT \\ Random KNN Modeling and Variable Selection for High Dimensional Data Shengqiao Li}

High dimensional data is widely available in bioinformatics, chemometrics and other applications. For example, in gene expression experiments, tens of thousands of genes are probed. Phenotype data may be clinical data such as tumor types, or quantities measuring biological characteristics of a subject. While such high dimensional data can be readily generated, successful analysis and modeling of these data is highly challenging.

Random KNN, as proposed in this dissertation, is a novel generalization of traditional nearest-neighbor modeling. Random KNN consists of an ensemble of base $\mathrm{k}$ nearest-neighbor models, each taking a random subset of the input variables. A theoretical and empirical analysis of the performance of the Random KNN is performed. Based on the proposed Random KNN, a new feature selection method is devised. To rank the importance of the variables, a criterion, named support, is defined and computed on the Random KNN framework. A two-stage backward model selection method is developed using supports. The present study shows that the Random KNN is a more effective and more efficient model for high-dimensional data than existing approaches.

The Random KNN approach can be applied to both qualitative and quantitative responses, i.e., classification and regression problems, and has applications in statistics, machine learning, pattern recognition and bioinformatics, etc.

Keywords: classification, regression, feature selection, bioinformatics, gene expression analysis 


\section{ACKNOWLEDGEMENT}

Pondering over this document, I have to say that the $\mathrm{PhD}$ study is one of the most important stages of my life. I enjoyed the pleasure of discovery and progress of this study and also faced the hardship of a higher level study, especially while working on a full job at the same time. It is the great help from my advisors Dr. Donald A. Adjeroh and Dr. E. James Harner that made the accomplishment of this study possible. I would like to take this opportunity to express my sincere gratitude to both of them. During my study, they provided an inspiring environment, constructive comments and constant encouragement that were indispensable for me. I am also thankful to Dr. Harner for his strong supports to my career in the past six years and also thank Dr. Adjeroh and Dr. Harner for their supports of my future career.

I also want to thank my committee members, Dr. Elaine M. Eschen, Dr. Robert Mnatsakanov, and Dr. Arun Ross for their very thoughtful discussions and suggestions. My special thanks go to Dr. Eschen for her careful coordination on the CCDM $\mathrm{PhD}$ program, which has proven to be successful. Clear step-by-step instructions were provided by her in a timely manner.

I am also grateful to my family, especially my mother and my wife, for their love, care and support. 
We learn by example and by direct experience because there are real limits to the adequacy of verbal instruction.

— Malcolm Gladwell, Blink: The Power of Thinking Without Thinking, 2005 


\section{Contents}

List of Figures viii

List of Tables $\quad$ X

$\begin{array}{lll}1 & \text { Introduction } & 1\end{array}$

1.1 Background . . . . . . . . . . . . . . . . . . . . 1

1.2 Our Approach . . . . . . . . . . . . . . . . . . 5

1.3 Data Sets Used . . . . . . . . . . . . . . . . . . . . . 7

1.4 Contributions . . . . . . . . . . . . . . . . . 7

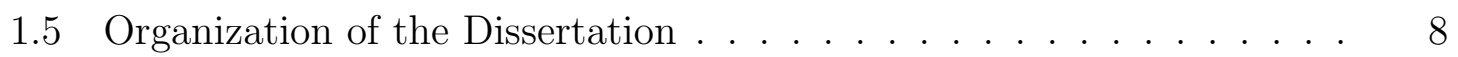

\begin{tabular}{lll}
\hline 2 & Random KNN & 9
\end{tabular}

2.1 Random Forests . . . . . . . . . . . . . . . . . . . . . . . . . . . . . . . $\quad 9$

2.2 Random KNN . . . . . . . . . . . . . . . . . . . . . . . . . . . . . . . 12

2.3 Properties of Random KNN . . . . . . . . . . . . . . . . . . . . 12

$2.3 .1 \quad$ Feature Coverage . . . . . . . . . . . . . . . 13

2.3 .2 Convergence of Random KNN . . . . . . . . . . . . . . . . . . 18

2.4 Complexity of Random KNN . . . . . . . . . . . . . . . . . . . 25

$2.4 .1 \quad$ Nearest Neighbor Searching Problem . . . . . . . . . . . . . . 25

2.4 .2 Complexity of Random KNN . . . . . . . . . . . . . 25 
2.5 Parameter Setting. . . . . . . . . . . . . . . . . . . . . . . . . . . . . 29

$2.5 .1 \quad$ Number of nearest neighbors, $k \ldots \ldots \ldots$

2.5 .2 Number of features,$m \ldots \ldots \ldots \ldots$

2.5 .3 Number of random KNN's, $r \ldots \ldots \ldots \ldots$

$2.6 \quad$ Cancer Gene Expression Data Sets $\ldots \ldots \ldots \ldots$

2.7 Application of Random KNN to Leukemia Microarray Data $\ldots . . .37$

3 Feature Selection with Random KNN 41

$3.1 \quad$ Bidirectional Voting and Variable Ranking . . . . . . . . . . . . . 41

3.1.1 Complexity of Computing Feature Support . . . . . . . . . 42

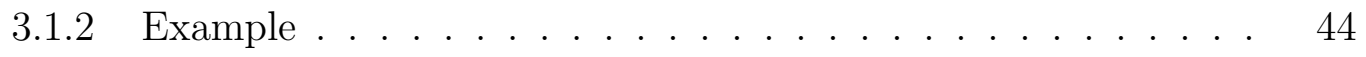

3.2 Feature Selection with Random KNN . . . . . . . . . . . . . . 45

$3.2 .1 \quad$ Complexity $\ldots \ldots \ldots \ldots \ldots \ldots$

$3.2 .2 \quad$ Example . . . . . . . . . . . . . . . . . . . . . . 48

3.3 Comparisons with other methods $\ldots \ldots \ldots \ldots \ldots$

3.4 Testing Random KNN on Other Data Sets . . . . . . . . . . . 52

3.4 .1 Test on Cancer Gene Expression Data Sets . . . . . . . . . . 52

3.4 .2 Detailed Testing Results . . . . . . . . . . . . . . . 53

$3.5 \quad$ Stability of Random KNN $\ldots \ldots \ldots \ldots \ldots \ldots$

3.6 Discussion $\ldots \ldots \ldots \ldots \ldots \ldots$

\begin{tabular}{|lll}
4 & Random KNN Regression & 60
\end{tabular}

$4.1 \quad$ Regression for High Dimensional Data $\ldots \ldots \ldots \ldots$

4.2 Random KNN Regression $\ldots \ldots \ldots$

$4.3 \quad$ Application of Random KNN Regression to Drug Sensitivity . . . . . 63

$4.4 \quad$ Feature Selection for Random KNN Regression $\ldots \ldots \ldots \ldots$

$4.4 .1 \quad$ Feature Ranking by Random KNN Regression . . . . . . . . . 65 
4.4 .2 Feature Selection for Random KNN Regression . . . . . . . . 66

4.5 Comparison with the LASSO . . . . . . . . . . . . . . . . . . . . 70

$\begin{array}{lll}5 & \text { Conclusions and Future Work } & 73\end{array}$

5.1 Major Contributions . . . . . . . . . . . . . . . . . 73

5.2 Limitations . . . . . . . . . . . . . . . . . . . . . . 74

5.3 Future Research Directions . . . . . . . . . . . . . . . . . . . . . . . . 74

5.4 Conclusions . . . . . . . . . . . . . . . . . . 75

\begin{tabular}{ll}
\hline Bibliography & 76
\end{tabular} 


\section{List of Figures}

2.1 Error rate convergence with $r \ldots \ldots \ldots \ldots \ldots$

$2.2 \quad$ Minimum error rates of Random KNN with different $\alpha$ and $\beta \quad \ldots \quad 23$

$2.3 \quad$ Error rates of one KNN with different $\alpha$ and $\beta \ldots \ldots \ldots . \ldots 24$

$2.4 \quad$ Random KNN speedup for simulated data, $m=\lfloor\sqrt{p}\rfloor \ldots \ldots \ldots . .27$

$2.5 \quad$ Random KNN speedup for simulated data, $m=\left\lfloor\log _{2}(p)\right\rfloor \ldots \ldots . .27$

$2.6 \quad$ Random KNN speedup for microarray data, $m=\lfloor\sqrt{p}\rfloor \ldots \ldots \ldots . \quad 28$

$2.7 \quad$ Random KNN speedup for microarray data, $m=\left\lfloor\log _{2}(p)\right\rfloor \ldots \ldots .28$

$\begin{array}{lll}2.8 & \text { Random KNN accuracies for different number of nearest neighbors, } k & 31\end{array}$

$2.9 \quad$ Random KNN accuracies for different feature subset size, $m(r=$ $500, k=3,30$ runs $) \ldots \ldots \ldots \ldots \ldots \ldots \ldots \ldots$

2.10 Random KNN accuracies for different number of KNN's, $r \ldots \ldots$

2.11 Histogram of the feature multiplicities $\ldots \ldots \ldots$

$3.1 \quad$ Diagram of bidirectional voting of random KNN $\ldots \ldots \ldots \ldots$

3.2 Supports of the first 30 genes of the Golub leukemia data . . . . . . 45

$3.3 \quad$ Mean accuracy change with the number of features for Golub leukemia data in the first stage . . . . . . . . . . . . . . . 48

$3.4 \quad$ Mean accuracy change with the number of features for Golub leukemia data in the second stage (peak feature set is selected) . . . . . . . . . 49 
3.5 Box plots of Random KNN accuracy before feature selection . . . . . 56

$3.6 \quad$ Box plots of Random KNN accuracy after feature selection . . . . . . 57

4.1 Supports of the top 30 genes of the NCI60 drug response data . . . . 67

4.2 Mean accuracy change with the number of features for NCI60 drug data 68

4.3 Mean accuracy change with the number of features for NCI60 drug data 69

4.4 Diagnostic plots for KNN regressions (9 genes for reduced model and $1,517$ genes for full model $)$. . . . . . . . . . . . . . . . . . 70

$4.5 \quad$ Diagnostic plots for the 13-variable linear model selected by LASSO . 71 


\section{List of Tables}

2.1 Numerical Values of some properties of Random KNN . . . . . . . . . 18

$2.2 \quad$ Average run time for KNN and Random KNN (seconds) . . . . . . . 26

2.3 Cancer microarray data sets . . . . . . . . . . . . . . . . . 35

$3.1 \quad$ Four genes selected by Random KNN for lung cancer survival prediction 50

3.2 Classification results of machine learning algorithms on lung cancer survival . . . . . . . . . . . . . . . . . . . 51

3.3 Classification accuracies (\%) on microarray data sets . . . . . . . . 53

3.4 Coefficient of variability of Random KNN classification accuracies of 30 runs . . . . . . . . . . . . . . . . . . . . 57

3.5 Accuracies of PCA-KNN classification on microarray data sets . . . . 59

4.1 Comparison of Random KNN regression with LASSO . . . . . . . . . 72 


\section{Chapter 1}

\section{Introduction}

\section{$1.1 \quad$ Background}

With advances in biotechnology, semiconductor technology, and computing science, explosive amounts of data are being generated. Retrieving useful information from this massive amount of data has become a major task in statistics, computer science, mathematics and the specific field related to the domain of the data. For example, QSAR (Quantitative Structure-Activity Relationship) is a computational process that relates the structure of a molecule to its chemical properties or biological activities. These properties provide crucial knowledge for occupational safety, industrial hygiene, health care, etc. In manufacturing, workers may be exposed to various chemicals. If we know their toxicity or sensitivity to humans, manufacturers can more effectively protect their workers. Similarly, in high throughput drug screening, chemicals for drug candidates are sifted out in a cost-saving manner by computing the bioactivity from chemical structure descriptors and other related data. In these types of applications, thousands of variables are often involved or available to scientists.

Recently, DNA chip/microarray technology has found a broad range of appli- 
cations and has become an indispensable tool for biologists and geneticists. The high-throughput microarray technique facilitates to profile whole genome expression simultaneously. On a DNA chip, there may be tens of thousands of probes/genes, each of which may relate to a specific biological process or function (one ontological term). These probes can hybrid with RNA samples obtained from cell specimens. In such data sets, each probe is a random variable, and scientists may be interested in finding genes related to a tumor, the stages of a tumor, or other phenotypes (continuous or discrete). The outcome or the dependent variable may be categorical (a class label) or quantitative, e.g., blood lipid panel, serum glucose level, tumor size, drug sensitivity, etc. The dimensionality of such data set is usually high.

Modeling, or learning, from high dimensional data is challenging. For microarray data, because of cost and other objective restrictions, the number of samples, $n$, is typically far less than the number of variables, $p$. This is sometimes referred to as the "small $n$, large $p$ " problem. A model cannot be built directly since the model complexity is larger than that of the data set. For example, technically, least-squares regression can fit a linear model up to $n$ parameters. Such models would provide a perfect fit, but has no prediction power. This problem essentially imposes extra difficulty to modeling practice, and the curse of dimensionality ${ }^{12159}$ is much more severe. In other words, the curse of dimensionality manifests itself in finding accurate estimates for model parameters, whether for classification or regression.

On the other hand, in gene expression data, most of the genes may not be related to (or useful in predicting) the target phenotype; thereby, the few "important" genes should be spotted and the other noisy genes should be excluded. Therefore, to efficiently build a good predictive model, or to determine the gene signature for a tumor, necessitates a serious look at dimension reduction or feature selection.

Principal Component Analysis (PCA) is one method of dimension reduction, in 
which the first few orthogonal principal components are expected to account for most of the variability. But this method is largely for visualization of the data. Since each principal component is a linear transformation of all the original variables, all the variables are still involved whether they are relevant or not.

In contrast, variable (feature) selection has more benefits which include ${ }^{110}$ : (i) reducing the complexity of computation; (ii) removing information redundancy (cost savings); (iii) avoiding the issue of overfitting; (iv) easing interpretation. In general, the more features we include, the worse is the generalization error. The higher the number of samples per parameter, the better. This is sometimes referred to as $O c$ cam's razor principle ${ }^{39}$. A complex model is hard to understand, so a parsimonious model is desired.

Different techniques have been developed to tackle the above issues. Basically, they can be grouped into three classes:

Class I: Classifiers or regression methods with internal variable selection. This class mainly consists of the Decision Trees $(\mathrm{DT})^{26}$, in which a variable is selected and split at each node by maximizing the purity of its descendant nodes. The variable selection process is done in the tree building process. The decision tree has the advantage of being easy to interpret, but it suffers from the instabilities of hierarchical structures. Errors from ancestors pass to multiple descendant nodes and thus have an inflated effect. Even worse, a minor change in the root may change the tree structure significantly. A relatively newer method is the Random Forest 2123 method, which grows a collection of trees by bootstrapping the samples and using a random selection of the variables. This approach decreases the prediction variance of a single tree. But the random forest may not remove a specific variable, as it may appear in another tree.

Class II: Variable filtering $20|54| 71$. This class encompasses a variety of filters 
that are principally used for the supervised learning problem, i.e. classification. A specific type of model may not be invoked in the filtering process. A filter is a statistic defined on a random variable over multiple populations. With the choice of a threshold, some variables can be removed. Such filters include $t$-statistics, $F$ statistics, Kullback-Leibler divergence, Fisher's discriminant ratio, mutual information $^{[5] 90[121122}$, information-theoretic networks ${ }^{72}$, maximum entropy ${ }^{104}$, Las Vegas Filter $(\mathrm{LVF})^{78}$ (based on Las Vegas probabilistic search), FINCO filters ${ }^{3}$, relief ${ }^{66169}$, correlation-based filters $^{1956 / 57 / 123}$, relevance and redundancy analysis ${ }^{65 \mid 123}$, etc. Whitely proposed an unsupervised correlation-based filter ${ }^{118}$ and Mitra proposed an unsupervised filer based on the maximum information compression index ${ }^{82}$; Xing et al. 120 used a mixture model, information gain, and a Markov blanket filter.

Class III: Optimal searching based on some criterion defined for a model. These techniques are also called wrapped methods ${ }^{20168}$ (wrap a model into a search algorithm). This class includes foreward/backword, stepwise selection for regression based on partial $F$-statistics for nested models. Other criteria include AIC (Aikaike's Information Criterion) $)^{4}$ and BIC (Bayesian Information Criterion) ${ }^{98}$. Both $\mathrm{AIC}$ and BIC penalize the number of parameters, $k$, estimated in the model:

$$
A I C=2 k-2 \ln L
$$

and

$$
B I C=k \ln n-2 \ln L,
$$

where $L$ is the likelihood. Under normality and independency assumptions, AIC and $\mathrm{BIC}$ are estimated by:

$$
\widehat{A I C}=2 k+n \ln (R S S / n)
$$


and

$$
\widehat{B I C}=k \ln n+n \ln (R S S / n)
$$

where $R S S$ is the residual sum of squares.

Zhai et al. $\frac{126}{1}$ combined sequential projection pursuit (SPP) and partial least square (PLS) analyses to select variables. Other search methods include floating

$\operatorname{search}^{92} \sqrt{95}$, adaptive floating search ${ }^{103}$, genetic algorithms ${ }^{48}$, simulated annealing ${ }^{967}$, branch and bound $\sqrt{86}$, hill climing $\sqrt{89}$, etc.

The advantage of the filter approaches is that they are simple to compute and very fast. They are good for pre-screening, rather than building the final model. On the contrary, Class III approaches are used to build the final model, but are slow and not scalable to many variables.

\subsection{Our Approach}

There are no easy technical solutions to the above problems, though various strategies have been developed. Earlier, we proposed a variable selection scheme ${ }^{76}$ for SVM (Support Vector Machine) $)^{27|4351 / 55 / 74| 116}$, which combines filter and wrapper approaches. In this dissertation, we will develop a new Class III method that includes a new classification and regression method containing a variable selection approach. Our method, called Random KNN, is based on the $k$-nearest neighbor (KNN) method. A collection of KNN models will be fitted with randomly selected variables. Compared to other models, KNN has many advantages.

KNN is a nonparametric classification method. It does not assume any parametric form for the distribution of measured random variables for a population. Due to the flexibility of the nonparametric model, it is usually a good classifier for many 
situations in which the joint distribution is hard to model parametrically. This is especially the case for DNA chips where it is common to have thousands of variables involved initially. Another advantage of KNN is that missing values can be easily imputed ${ }^{[229160 / 112}$, and it is more robust and sensitive than other methods ${ }^{112}$.

The mechanism of KNN is very straightforward compared to other methods. KNN uses instance based reasoning. The philosophy of nearest neighbor classification is that two subjects in the same class have some kind of similarity, measurable by a distance metric through some variables, and vice versa. An item, with an unknown class, is classified into the same category as its first nearest neighbor or the wining class by the votes of its $k$ nearest neighbors, where $k$ is usually an odd number, such as $1,3,5$ or 7 , etc. This $k$ is usually chosen by cross-validation. Nearest neighbors are found by a distance or dissimilarity measure. Therefore, the design of nearest neighbor classifiers involves three key elements: a distance metric, $d$; the number of nearest neighbors, $k$; and the selection of a set of variables for distance computation. Compared with Random Forest, Random KNN uses stable base KNN's, thereby no hierarchical structure is involved. Hence, Random KNN can be stabilized with a small number of base KNN's and thus only a small number of important variables may be needed. Therefore, the final model with Random KNN will be simpler than that with Random Forest.

The Euclidean distance is widely used and can be generalized to the Minkowski distance, $L_{q}$ norm. Let $\mathrm{v}$ be the difference vector between vector $\mathbf{x}$ and $\mathbf{y}$. Then $L_{1}$ is the Manhattan distance or City-Block distance, $L_{2}$ is the Euclidean distance, and $L_{\infty}$ is the maximum of the absolute value of each element of $\mathbf{v}$. Minkowski distances make sense when there is commensurability between different variables. The aforementioned distance metrics do not take into account the correlation in the variable space. Another type of distance, the Mahalanobis distance, generalizes the 
Euclidean distance in another way, and factors in the correlations between variables in the distance calculation. The Mahalanobis distance is defined as $\operatorname{Mah}(\mathbf{v})=\sqrt{\mathbf{v}^{\prime} \mathbf{M v}}$ where $M$ is a positive semi-definite $p \times p$ matrix. If $\mathbf{M}$ is an identity matrix, the distance is just the usual Euclidean distance. If the inverse of the variance-covariance matrix $\mathbf{S}$ is taken as $\mathbf{M}$, the Mahalanobis distance results. Actually, Mahalanobis distances not only account for the dependency, but also standardize the measure units. Thus, sometimes it is preferable.

In addition to the distance metrics discussed above, researchers have also proposed more complicated measures based on those for the nearest neighbor algorithm, such as, local distance measure, weighted distance ${ }^{102}$, adaptive method ${ }^{61}$, etc. In this study, we use the Euclidean distance for simplicity.

\subsection{Data Sets Used}

In this study, ten microarray and one chemometrics data sets are used to demonstrate and test the proposed methods. Besides, simulated data are also used for testing the speed of the proposed methods. Details of these data sets will be provided in the following chapters.

\subsection{Contributions}

In this study, a novel generalization of traditional nearest-neighbor modeling, Random $\mathrm{KNN}$, is proposed. A theoretical and empirical analysis of the performance, and some properties of the Random KNN are developed. Based on the proposed Random KNN, a new feature selection method is devised. To rank the importance of the variables, a criterion, named support, is defined and computed on the Random KNN 
framework. A two-stage backward model selection method is developed using the support criterion. The present study shows that the random KNN is a more effective and more efficient model for high-dimensional data than existing approaches.

The Random KNN approach can be applied to both qualitative and quantitative responses, i.e., classification and regression problems, and has applications in statistics, machine learning, pattern recognition and bioinformatics, etc.

\subsection{Organization of the Dissertation}

This dissertation is organized as follows: In chapter 2, the Random KNN method will be explained and some of its properties will be studied. Random KNN classification will be illustrated using microarray data. In chapter 3, a variable selection approach, based on Random KNN classification, is proposed. The algorithm is described and applied to the same cancer microarray data set used in chapter 2. Preliminary tests and comparisons are also done on other data sets. Chapter 4 examines regression, and related variable selection problems, using Random KNN. Chapter 5 gives conclusions and speculates on future directions on the topics studied in this dissertation. 


\section{Chapter 2}

\section{Random KNN}

The idea for Random KNN is motivated by Random Forests $(\mathrm{RF})^{21 / 23}$, which was developed by Leo Breiman. The next section gives some background on RF.

\subsection{Random Forests}

The Random Forest algorithm grows a collection of classification trees, called a forest, and uses these for classifying a data point into one of the classes. Two types of randomness: bootstrap sampling and random selection of input variables, are used in the algorithm to ensure that the classification trees grown in the forest are dissimilar and uncorrelated from each other. Growing a forest of trees, and using randomness in building each classification tree in the forest, leads to better predictions as compared to a single classification tree, and helps to make the algorithm robust to noise in the data set.

The general methodology of developing a random forest is: A forest is grown by using $B$ bootstrapped samples, each of size $n$, randomly drawn with replacement from the original sample of $n$ points. This first type of randomization (bootstrap 
sampling) helps in building an ensemble of trees and in reducing the dependence among the trees. About two-thirds (more precisely, $1-\left(1-\frac{1}{n}\right)^{n}$ ) of the data set are used to grow a classification tree. The left-out observations (about one-third), called Out-Of-Bag $(O O B)$ data, is used for obtaining unbiased estimates of correct classification rates and for variable importance used for ranking variables. The second type of randomness (random selection of the input variables) is used while building the classification trees. For each node of a tree, the RF algorithm randomly selects $m$ variables and uses them to determine the best possible split using the Gini index ${ }^{26 \mid 62}$ as the splitting criterion. This algorithm is fairly robust to the choice of $m$. Usually $m=\sqrt{p}$, where $p$ is the total number of variables ${ }^{108}$. In contrast to the classification tree approach, random forest trees are grown to full height and are not pruned back. Predictions for test data are carried out either by the majority vote of the classification trees in the forest, or are based on a threshold value selected by the user. The number of trees to be grown in the forest is chosen to achieve low error rate.

When applying the random forest algorithm, it is not necessary to set aside a portion of data as test data set or to use cross-validation to estimate correct classification rates. Instead, the $O O B$ part of the data is used. Each tree in the forest is grown using a bootstrapped sample and the $O O B$ part is subsequently processed by the grown tree. This gives rise to a classification for each point in the $O O B$ part of that bootstrapped sample. This means that about one third of the trees in the random forest provide a prediction for each point in the original data. The final classification of a particular data point is decided on the basis of a majority vote or a user-defined threshold, among the trees predicting that data point. The unbiased estimates of true classification rates are calculated by comparing the $O O B$ classifications made by the forest to the experimentally observed classes to which the data points belong.

The random forest algorithm can also give two measures of importance for the 
variables used in growing trees. The first measure, the Mean Decrease in Accuracy, uses the $O O B$ part of the data set. Values of a variable $X_{i}$ in the $O O B$ part are randomly permuted and the resulting new $O O B$ data points are processed by the random forest. The margin of a data point is defined as the difference between the proportion of the votes for the correct class and the proportion of votes for the other class. The percent decrease in the margin, resulting from the random permutation in the values of the variable $X_{i}$ averaged across all data points in the $O O B$ set, gives the first measure of importance of variable $X_{i}$. The second importance measure, the Mean Decrease in Gini, is based on the Gini index, also used as the splitting criterion. The value of the Gini index decreases as a result of each split, based on one of the mtry variables chosen randomly. Therefore, the second measure of variable importance for variable $X_{i}$ can be defined as the sum of all decreases of the Gini index in the forest due to this variable divided by the total number of trees in the forest. The first measure of importance is considered more reliable than the second measure ${ }^{22}$. In general, a variable is considered important if at least one of the above measures has a large value.

One problem with RF is that the prediction relies on all the input variables. There are no best subsets of variables reported. So we designed a two-stage backward elimination approach and applied it to a QSAR study ${ }^{75}$ in 2005. A similar method was also studied by others in $2006^{32}$. Even with these extensions, in order to stable the hierarchical tree structures, RF may need to keep a relative large number of variables to generate many dissimilar trees.

Various authors have studied Random Forests from different perspectives. For instance, Lin and Joen discovered the connections between Random Forests and adaptive nearest neighbor methods, which use adaptive local distance metrics ${ }^{77}$. 


\subsection{Random KNN}

The basic strategy used in Random Forests, i.e., collections of classifiers and randomization, can be applied to other supervised learners, such as the prominent $k$-Nearest Neighbor (KNN) classifier. Compared with decision trees, KNN is simple to implement and is stable. A large number of different KNN classifiers will be generated. Each one takes a random subset of the input variables (features). Since KNN is stable ${ }^{38}$, bootstrapping is not necessary for KNN as Breiman suggested 25: "Bagging stable classifiers is not a good idea." Each KNN classifier classifies a testing point by its majority, or weighted majority class, of its $k$ neighbors. The final classification in each case is determined by majority voting of $r$ KNN classifications. This is sort of a Majority of a Majority.

More formally, let $\mathbf{F}=\left\{f_{1}, f_{2}, \ldots, f_{p}\right\}$ be the $p$ input features, and $\mathbf{X}$ be the $n$ original input data vectors of length $p$, i.e., an $n \times p$ matrix. For a given integer $m<p$, denote $\mathbf{F}^{(m)}=\left\{f_{j_{1}}, f_{j_{2}}, \ldots, f_{j_{m}} \mid f_{j_{l}} \in \mathbf{F}, 1 \leq l \leq m\right\}$ a random subset drawn from $\mathbf{F}$ with equiprobability. Similarly, let $\mathbf{X}^{(m)}$ be the data vectors in the subspace defined by $\mathbf{F}^{(m)}$, i.e., an $n \times m$ matrix. Then a random $\mathbf{K N N}{ }^{(m)}$ can be built based on data $\mathbf{X}^{(m)}$. A collection of $r$ such base classifiers can be built as the final classifier.

\subsection{Properties of Random KNN}

In this section, some probability properties related to Random KNN method, such as feature coverage, number of random KNN's to be generated and convergence, etc., will be discussed. 


\subsubsection{Feature Coverage}

The coverage of the classifier over the input features will be studied. The coverage essentially indicates whether all features are used in the collection of $\mathbf{K N N}^{(m)}$ and for what size of Random KNN, $r$, all these features will be covered.

\section{A. Feature Multiplicity}

All variables that appear in the set of classifiers constitute a multiset. Define the random variable multiplicity, $M$, of an input feature as the number of times that feature appears in the multiset. Then $M$ is a binomial random variable with probability $m / p$, i.e., $M \sim B I N(r, m / p)$, and the average multiplicity of a feature $f$, the $\nu$ number, is given by:

$$
\nu=\mathbb{E}(M)=\frac{r m}{p} .
$$

\section{B. Silent Features}

A feature $f$ may not be selected by any of the KNN classifiers and thus it has no voice in classification decisions. We call such a feature a silent feature. The chance of a feature being silent will be small if $m$ and $r$ are sufficiently large. Specifically, define a binary indicator variable, $I_{f}$, to indicate if a feature $f$ is silent $\left(I_{f}=1\right.$ when feature $f$ is silent). The probability of $f$ being silent is then given by:

$$
P\left(I_{f}=1\right)=P(M=0)=\left[\left(\begin{array}{c}
p-1 \\
m
\end{array}\right) /\left(\begin{array}{c}
p \\
m
\end{array}\right)\right]^{r}=\left(1-\frac{m}{p}\right)^{r} .
$$

If we let $S$ be the number of silent features, i.e., $S=\sum_{f} I_{f}$, then $0 \leq S \leq p-m$. Since the $I_{f}$ 's are dependent, $S$ is not a binomial random variable. In fact, $S$ is the same as the number of coupons not yet collected in the coupon collector's problem, 
while drawing a subset each time $\mathrm{C}^{6 / 47 / 80 \mid 105 / 106 / 117}$. The expectation of $S$ and probability function are given by the following 106 :

$$
\lambda=\mathbb{E}(S)=p\left(1-\frac{m}{p}\right)^{r}
$$

and

$$
\begin{aligned}
P(S=s) & =\left(\begin{array}{c}
p \\
s
\end{array}\right) \sum_{j=0}^{p-s}(-1)^{j}\left(\begin{array}{c}
p-s \\
j
\end{array}\right)\left(\begin{array}{c}
p-s-j \\
m
\end{array}\right)^{r} /\left(\begin{array}{c}
p \\
m
\end{array}\right)^{r} \\
& =\left(\begin{array}{c}
p \\
s
\end{array}\right) \sum_{j=0}^{p-s}(-1)^{p-s-j}\left(\begin{array}{c}
p-s \\
j
\end{array}\right)\left(\begin{array}{c}
j \\
m
\end{array}\right)^{r} /\left(\begin{array}{c}
p \\
m
\end{array}\right)^{r} .
\end{aligned}
$$

\section{Coverage probability}

The probability that all features are used at least once is defined as the coverage probability, $\eta$. That is,

$$
\begin{aligned}
\eta & =P(S=0) \\
& =\sum_{j=0}^{p}(-1)^{j}\left(\begin{array}{l}
p \\
j
\end{array}\right)\left[\left(\begin{array}{c}
p-j \\
m
\end{array}\right) /\left(\begin{array}{c}
p \\
m
\end{array}\right)\right]^{r} \\
& =\sum_{j=0}^{p}(-1)^{p-j}\left(\begin{array}{l}
p \\
j
\end{array}\right)\left[\left(\begin{array}{c}
j \\
m
\end{array}\right) /\left(\begin{array}{c}
p \\
m
\end{array}\right)\right]^{r},
\end{aligned}
$$

where $\left(\begin{array}{c}p \\ j\end{array}\right)\left[\left(\begin{array}{c}p-j \\ m\end{array}\right) /\left(\begin{array}{c}p \\ m\end{array}\right)\right]^{r}$ is the $j$ th binomial moment of $S$. The binomial coefficient $\left(\begin{array}{l}p \\ j\end{array}\right)$ in the above equations will overflow for large $p$ and thus the probability cannot easily be computed. In fact, to accurately compute the maximum binomial coefficient $\left(\begin{array}{c}p \\ p / 2\end{array}\right)$, the number of bits needed can be estimated using Stirling approximation $(n ! \approx$ 
$\left.\sqrt{2 \pi n}\left(\frac{n}{e}\right)^{n}\right)$ as follows:

$$
\begin{aligned}
\log _{2}\left(\begin{array}{c}
p \\
p / 2
\end{array}\right) & \approx \frac{1}{2} \log _{2} 2 \pi p+p \log _{2}(p / e)-\log _{2} \pi p-p \log _{2}(p / 2 / e) \\
& =p+\left(1-\log _{2} \pi p\right) / 2 \\
& \approx p
\end{aligned}
$$

Thus, $\left(\begin{array}{c}p \\ p / 2\end{array}\right) \approx 2^{p}$. It is difficult to represent such a big number by a standard data type on a computer for a large $p$. For example, the maximum 64-bit double precision floating number is $2^{1024}$. Therefore arbitrary-precision arithmetic is required. Here, we give a simple approximation for the case when $p$ is large and $m / p$ is small. Since:

$$
\left(\begin{array}{c}
j \\
m
\end{array}\right) /\left(\begin{array}{c}
p \\
m
\end{array}\right)=\left(1-\frac{m}{p}\right)\left(1-\frac{m}{p-1}\right) \ldots\left(1-\frac{m}{j+1}\right)<\left(1-\frac{m}{p}\right)^{p-j}
$$

we may ignore the dependency among $I_{f}$ 's, and approximate $S$ by a binomial random variable $B I N\left(p,(1-m / p)^{r}\right)$. Then we use $\eta_{b}$ as an approximation of $\eta$ and it is numerically justified that $\eta_{b}$ is an upper bound of $\eta$, i.e:

$$
\begin{aligned}
\eta & =P(S=0) \\
& <\sum_{j=0}^{p}(-1)^{p-j}\left(\begin{array}{l}
p \\
j
\end{array}\right)\left[\left(1-\frac{m}{p}\right)^{p-j}\right]^{r} \\
& =\sum_{j=0}^{p}(-1)^{p-j}\left(\begin{array}{l}
p \\
j
\end{array}\right)\left[\left(1-\frac{m}{p}\right)^{r}\right]^{p-j} \\
& =\left[1-\left(1-\frac{m}{p}\right)^{r}\right]^{p} \\
& =\eta_{b} .
\end{aligned}
$$


This bound is easy to compute. Another simple way to compute $\eta$ is to approximate $S$ by a Poisson random variable. Then

$$
\eta_{p}=e^{-\lambda}
$$

where $\lambda$ is defined by equation 2.3 .

\section{Random KNN Size}

The preceding discussion provides guidance for the number of KNN to generated, $r$. $r$ may be determined by inverting the binomial or Poisson approximation of $\eta$ if $\eta$ is pre-specified, respectively, as follows:

$$
r_{b}=\frac{\ln \left(1-\eta^{1 / p}\right)}{\ln (1-m / p)}
$$

or

$$
r_{p}=\frac{\ln (-\ln \eta)-\ln p}{\ln (1-m / p)} .
$$

If pre-specify $\nu, r$ can be derived by:

$$
r=\nu p / m
$$

On the other hand, we may consider a minimum number of $\mathrm{KNN}, R$, when each feature is drawn at least once. The mean, $r_{z}$, can be obtained ${ }^{[6]}$ by:

$$
r_{z}=\mathbb{E}(R)=\left(\begin{array}{c}
p \\
m
\end{array}\right) \sum_{j=1}^{p}(-1)^{j+1} \frac{\left(\begin{array}{c}
p \\
j
\end{array}\right)}{\left(\begin{array}{c}
p \\
m
\end{array}\right)-\left(\begin{array}{c}
p-j \\
m
\end{array}\right)} .
$$

This can be a reference value of $r$. In computation, the same problem occurs for a large binomial coefficient. One simple way is to simulate the distribution of $R$ and 
estimate its mean. As before, we will consider an approximate distribution of R. Let $R_{f}$ be the minimum $r$, until which the specific feature $f$ is drawn. Then $R_{f}$ is a geometric random variable with parameter $m / p$, i.e,

$$
P\left(R_{f} \leq r\right)=P\left(I_{f}=0\right)=1-\left(1-\frac{m}{p}\right)^{r}
$$

For $p$ features $f_{1}, f_{2}, \ldots$, and $f_{p}, R_{f_{1}}, R_{f_{2}}, \ldots$, and $R_{f_{p}}$ are identically distributed but dependent. $R$ is the maximum of these dependent geometric random variables. For $p \gg m$, the dependency is small. Ignoring the dependency and considering the maximum of i.i.d. geometric random variables, $R_{g}$, we have:

$$
P\left(R_{g} \leq r\right)=\left[1-\left(1-\frac{m}{p}\right)^{r}\right]^{p}
$$

and

$$
P\left(R_{g}>r\right)=1-\left[1-\left(1-\frac{m}{p}\right)^{r}\right]^{p}
$$

Thus,

$$
r_{z} \approx \mathbb{E}\left(R_{g}\right)=\sum_{r=0}^{\infty} P\left(R_{g}>r\right)=\sum_{r=0}^{\infty}\left\{1-\left[1-\left(1-\frac{m}{p}\right)^{r}\right]^{p}\right\}
$$

The above infinite series can be approximated by finite terms and can be easily computed.

Remarks: The geometric approximation of the mean of $R$ can also be derived by plugging the formula 2.7 into the equation 2.15. That is replacing $\left(\begin{array}{c}p-j \\ m\end{array}\right)$ by $\left(\begin{array}{c}p \\ m\end{array}\right)\left(1-\frac{m}{p}\right)^{j}$ :

$$
\mathbb{E}(R) \approx \sum_{j=1}^{p}(-1)^{j+1} \frac{\left(\begin{array}{l}
p \\
j
\end{array}\right)}{1-\left(1-\frac{m}{p}\right)^{j}}
$$


The RHS of the equation is recognized as the mean of the maximum of $p$ i.i.d geometric random variables with success probability $m / p^{81}$. Although this formula is equivalent to 2.19, the latter is easier to compute by eliminating the binomial coefficients as discussed.

\section{E. Numerical Values}

Table 2.1 listed some numerical values for above discussion. For the subset size drawn for each bass KNN, we choose $m=\lfloor\sqrt{p}\rfloor$, by default (see page 31 for the discussion of parameter $m$ ).

Table 2.1: Numerical Values of some properties of Random KNN

\begin{tabular}{l|l|l|l|l|l|l}
\hline$p$ & 10 & 10 & 100 & 1000 & 10000 & 10000 \\
$m$ & 3 & 3 & 10 & 31 & 100 & 100 \\
$r$ & 10 & 20 & 100 & 500 & 1000 & 1500 \\
\hline \hline$\nu$ & 3 & 6 & 10 & 15.5 & 10 & 15 \\
$\lambda$ & 0.28248 & 0.00798 & 0.00266 & 0.00015 & 0.43171 & 0.00284 \\
$\eta$ & 0.73904 & 0.99203 & 0.99735 & 0.99985 & overflow & overflow \\
$\eta_{b}$ & 0.75086 & 0.99205 & 0.99735 & 0.99985 & 0.64939 & 0.99717 \\
$\eta_{p}$ & 0.75392 & 0.99205 & 0.99735 & 0.99985 & 0.6494 & 0.99717 \\
\hline$r_{b}$ & 10 & 20 & 100 & 499 & 1000 & 1500 \\
$r_{p}$ & 10 & 20 & 100 & 499 & 1000 & 1500 \\
\hline$r_{z}$, by simulation & 9 & 9 & 50 & 238 & 974 & 974 \\
$r_{z}$, by $R_{g}$ & 9 & 9 & 50 & 238 & 974 & 974 \\
\hline
\end{tabular}

Table 2.1 shows that our approximations of $\eta$ are quite accurate and scalable for a very large $p$. For $r_{z}$, the approximation and simulation results agree with each other.

The above properties provide guidance for determining how many KNN's to be generated.

\subsubsection{Convergence of Random KNN}

In this section, we discuss the convergence of Random KNN for a simplified case where variables are indecent of each other. 
Theorem 2.3.1. (Convergence in $n$ ) Assume each KNN converges, then Random KNN converges for infinite sample size, $n$.

Proof. Cover \& Hart in 1967 proved that the KNN converges almost surely as the sample size $n$, increases with a given $k^{28}$. It is straightforward to show that Random KNN, based on the majority voting rule, also converges almost surely as the sample size $n$ increases.

Theorem 2.3.2. (Convergence in $r$ ) Suppose the $p$ variables are independent and equally important, Random KNN converges almost surely as the number of KNN's, $r$, increases.

Proof. Under the independence and equality of importance assumption, each KNN, with $m \ll p$ variables, is independent and has the same accuracy $\pi$ and error rate $1-\pi$. For $r$ KNN's, let $Y$ be the number of correct votes. Then $Y \sim B I N(r, \pi)$. By majority voting, the error rate is:

$$
\begin{aligned}
P e & =P(Y \leq r / 2) \\
& =\sum_{y=0}^{r / 2}\left(\begin{array}{l}
r \\
y
\end{array}\right) \pi^{y}(1-\pi)^{r-y} .
\end{aligned}
$$

We will apply the Chernoff bound to the tail distribution of $Y$. The Chernoff bound for Poisson trials (independent trials with different success probabilities) can be derived by applying the Markov inequality. See Mitzenmacher and Upfal's book ${ }^{83}$. By formula 4.5 on page 66 of this book, for $0<\delta<1$,

$$
P(Y \leq(1-\delta) \mu) \leq e^{-\mu \delta^{2} / 2}
$$

Bernoulli trials are special cases of Poisson trials, so the above Chernoff upper 
bound for the lower tail distribution also applies. With $\pi>\frac{1}{2}, \mu=\pi r$ and $\delta=1-\frac{1}{2 \pi}$ (thus $0<\delta<1$ ), we have:

$$
\begin{aligned}
P e & =P(Y \leq r / 2) \\
& \leq e^{-\frac{r}{2 \pi}\left(\pi-\frac{1}{2}\right)^{2}} \\
& \rightarrow 0, \text { as } r \text { increases. }
\end{aligned}
$$

Remarks: In the preceding theorem, the KNN error rate $\pi$ is assumed to be a fixed value. More generally, we may consider the error rate to be a random variable $\Pi$. For example, a $\operatorname{BETA}(\alpha, \beta)$ distributed random variable, where $\alpha$ and $\beta$ are shape parameters, is conjugate to binomial distributions. The shape parameters give the $B E T A$ distribution the flexibility to model a wide range of different distributions. Hence we may assume $\Pi \sim \operatorname{BETA}(\alpha, \beta)$ and the integrated error rate will be the overall error rate for Random KNN. Since

$$
P e(\pi)=P(Y \leq r / 2 \mid \Pi=\pi)=\sum_{y=0}^{r / 2}\left(\begin{array}{l}
r \\
y
\end{array}\right) \pi^{y}(1-\pi)^{r-y}
$$

then

$$
\begin{aligned}
P e & =\mathbb{E}_{\Pi}(P e(\pi))=\int_{0}^{1} \sum_{y=0}^{r / 2}\left(\begin{array}{l}
r \\
y
\end{array}\right) \pi^{y}(1-\pi)^{r-y} \cdot \frac{1}{B(\alpha, \beta)} \pi^{\alpha-1}(1-\pi)^{\beta-1} d \pi \\
& =\frac{1}{B(\alpha, \beta)} \sum_{y=0}^{r / 2}\left(\begin{array}{l}
r \\
y
\end{array}\right) \int_{0}^{1} \pi^{\alpha+y-1}(1-\pi)^{\beta+r-y-1} d \pi \\
& =\frac{1}{B(\alpha, \beta)} \sum_{y=0}^{r / 2}\left(\begin{array}{l}
r \\
y
\end{array}\right) B(\alpha+y, \beta+r-y),
\end{aligned}
$$


where $B$ is the beta function. Or:

$$
P e=\frac{1}{(r+1) B(\alpha, \beta)} \sum_{y=0}^{r / 2} \frac{B(\alpha+y, \beta+r-y)}{B(1+y, 1+r-y)} .
$$

The above equations are non-increasing functions of $r$.

For a special example, let $\alpha=2, \beta=1$, then

$$
\begin{aligned}
P e & =2 \sum_{y=0}^{r / 2} \frac{r !}{y !(r-y) !} \frac{(y+1) !(r-y) !}{(r+2) !} \\
& =2 \sum_{y=0}^{r / 2} \frac{y+1}{(r+1)(r+2)} \\
& =\frac{2}{(r+1)(r+2)} \sum_{y=0}^{r / 2}(y+1) \\
& =\frac{2}{(r+1)(r+2)} \frac{(r / 2+1)(r / 2+1+1)}{2} \\
& =\frac{1}{4} \frac{(r+2)(r+4)}{(r+1)(r+2)} \\
& =\frac{r+4}{4(r+1)} \\
& \rightarrow \frac{1}{4} .
\end{aligned}
$$

The error rate for one $m$-variable base $\mathrm{KNN}$ is $1-\mathbb{E}(\Pi)=1-\frac{\alpha}{\alpha+\beta}=\frac{1}{3}$, which is greater than the above Random KNN error rate.

Figure 2.1 shows that error rates decrease with $r$ for different $\alpha$ and $\beta$. Under the above conditions, the Random KNN converges fairly fast.

Figure 2.2 shows a 3 -D plot and a contour plot of the minimum error rates $(r=$ 200) for different $\alpha$ and $\beta$. We can see that the larger $\alpha$ is than $\beta$, the smaller the error rate. 


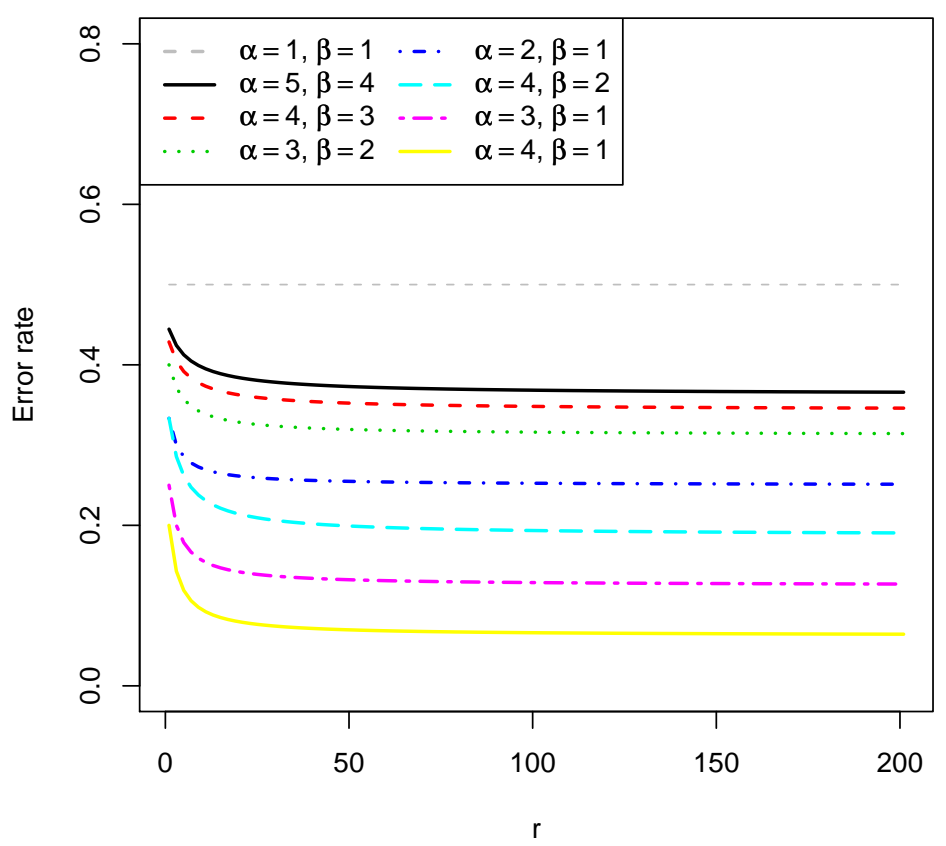

Figure 2.1: Error rate convergence with $r$

Figure 2.3 shows error rates for one KNN. Compared with the preceding figures, the error rates change more evenly.

Figures 2.2 and 2.3 demonstrate that better performances can be obtained by combining weak KNN classifiers. But if the base KNN classifiers are weaker than "by-chance", the resulting Random KNN will be worse than the base KNN's. 

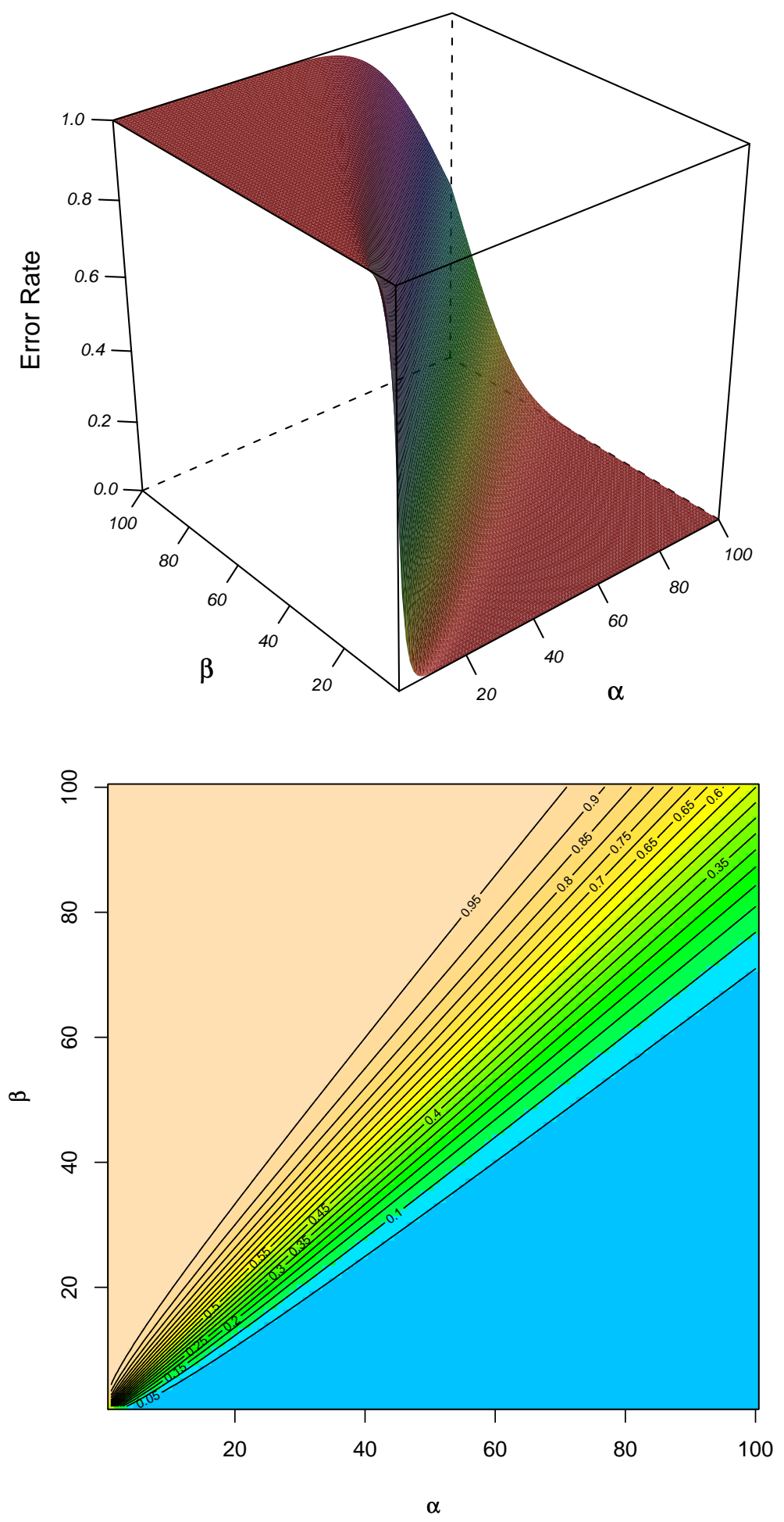

Figure 2.2: Minimum error rates of Random KNN with different $\alpha$ and $\beta$ 

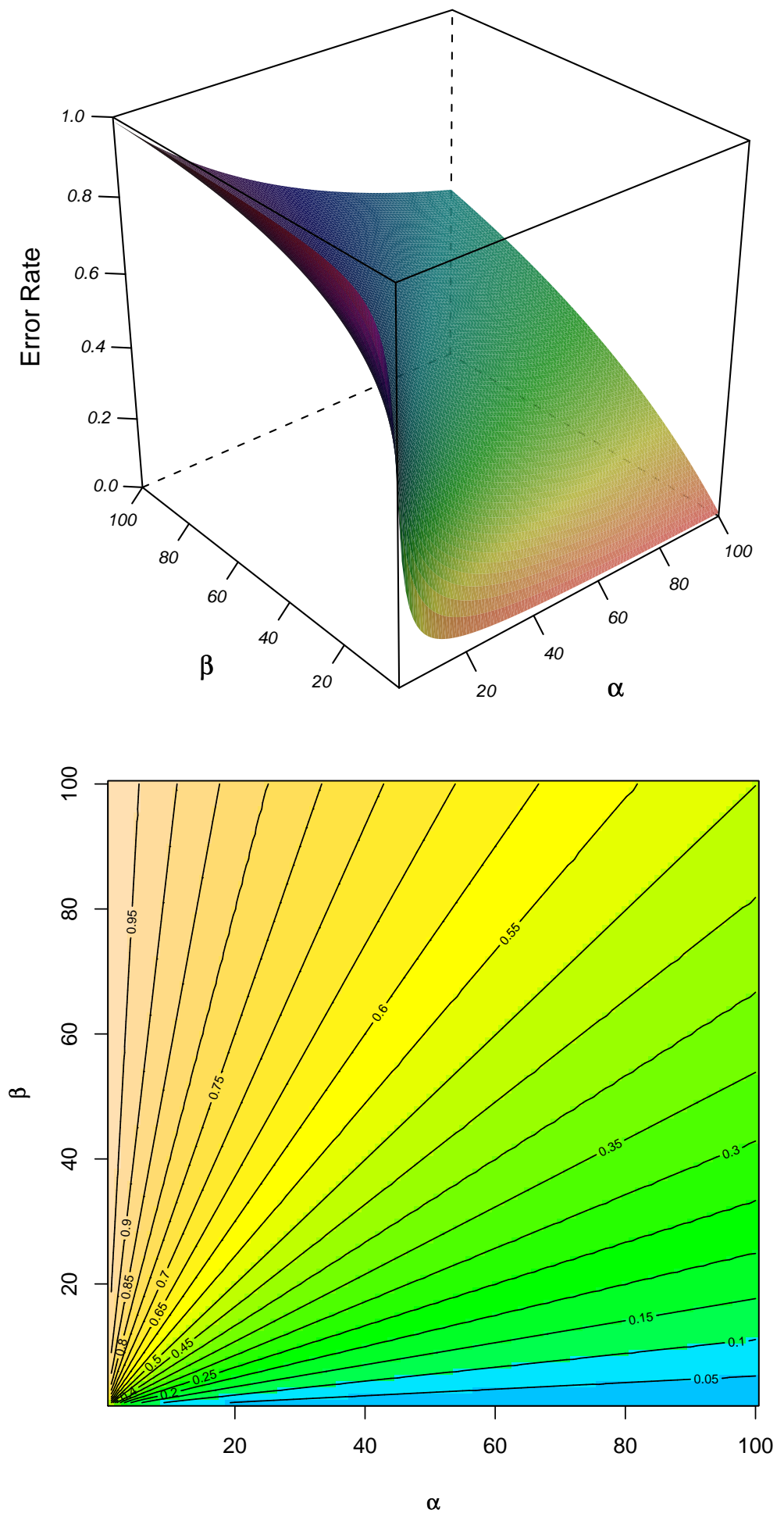

Figure 2.3: Error rates of one KNN with different $\alpha$ and $\beta$ 


\subsection{Complexity of Random KNN}

\subsubsection{Nearest Neighbor Searching Problem}

The time complexity of Random KNN classification is mainly determined by the underlying $k$ nearest neighbor searching algorithm. There are many such searching algorithms. Some of them are tree based, such as the $k$-dimensional binary

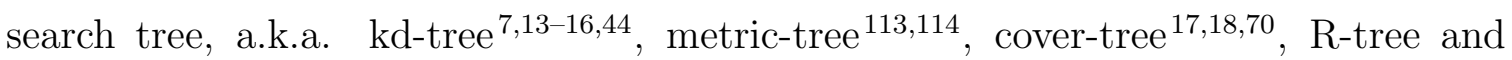
variants $^{10|53| 97 \mid 100}$, hB-trees ${ }^{79}$, quad-tree ${ }^{\sqrt{45}}$, etc. Some of them use hashing functions such as locality sensitive hashing $46[63$, geometric hashing $[119$, etc. These algorithms provide sublinear $(\log n)$ time searching through special data structures for a given data point and are very efficient for low dimensional data. In terms of dimensionality, most $k$-nearest neighbor searching algorithms have difficulty on high dimensional data and exhibit exponential complexity behaviors and thus are not efficient for high dimensional data. There have been efforts to improve performance in high dimensional data, but usually some degree of accuracy is sacrificed (approximate nearest-neighbor approaches), such as BoostMap ${ }^{8}$ and slicing $\sqrt{88}$, etc.

\subsubsection{Complexity of Random KNN}

Assuming a run-time bound of $O\left(2^{p} k n \log n\right)$ for KNN searching with a reasonable implementation of any one of the aforementioned algorithms, then the time for Random KNN will be $O\left(r 2^{m} k n \log n\right)$. Take $m=\sqrt{p}$. Then the complexity becomes $O\left(r 2^{\sqrt{p}} k n \log n\right)$. Since the exponent is much smaller than that for the ordinary KNN method, Random KNN will be much faster for high dimensional data.

We compared the running time of KNN with our Random KNN on a Dell Desktop OptiPlex 745 with 3G Hz Pentium D CPU and 4GB RAM. The software was run in 
$R$ 2.9.0 under Windows XP (service pack 3) operating system. A measure of relative speed is defined as:

$$
\text { speedup }=\frac{T_{K N N}}{T_{R K N N}}
$$

where $T_{K N N}$ is the average running time of 100 runs of KNN and $T_{R K N N}$ is the average running time of 100 runs of Random KNN. For Random KNN, we take either $m=\lfloor\sqrt{p}\rfloor, \nu=2$, and $r=2\lfloor\sqrt{p}\rfloor$ or $m=\left\lfloor\log _{2} p\right\rfloor, \nu=2$, and $r=2\left(p / \log _{2} p\right)$. To test speed, we simulated a standard multivariate normal distribution with mean 0 for one class and mean 2 for another class. Figures 2.4 and 2.5 show Random KNN speedup against the number of features on simulated data. Timing for left panels is only for nearest neighbor searching and that for right panels is for overall running time. Similarly, Figure 2.6 and 2.7 show testing results on 8 microarray data sets. From these figures, Random KNN is significantly faster than KNN when the number of features is large. So our method is suitable for high dimensional data, such as microarray data.

In Table 2.2, running time on the simulated data is listed. One can see that the current implementation of the Random KNN algorithm is fast.

Table 2.2: Average run time for KNN and Random KNN (seconds)

\begin{tabular}{l|ll|ll|ll}
\hline \multirow{2}{*}{$\mathrm{p}$} & \multicolumn{2}{|c|}{$\mathrm{n}=25$} & \multicolumn{2}{c|}{$\mathrm{n}=50$} & \multicolumn{2}{c}{$\mathrm{n}=75$} \\
\cline { 2 - 7 } & KNN & RKNN & KNN & RKNN & KNN & RKNN \\
\hline \hline 1000 & 0.01 & 0.09 & 0.06 & 0.19 & 0.15 & 0.32 \\
2000 & 0.03 & 0.12 & 0.12 & 0.26 & 0.36 & 0.46 \\
3000 & 0.04 & 0.15 & 0.22 & 0.33 & 0.58 & 0.61 \\
4000 & 0.07 & 0.17 & 0.35 & 0.41 & 0.77 & 0.76 \\
5000 & 0.12 & 0.20 & 0.44 & 0.48 & 1.00 & 0.88 \\
6000 & 0.18 & 0.23 & 0.75 & 0.55 & 1.65 & 1.02 \\
7000 & 0.31 & 0.25 & 1.20 & 0.61 & 2.82 & 1.15 \\
8000 & 0.50 & 0.27 & 1.87 & 0.68 & 4.99 & 1.32 \\
9000 & 0.78 & 0.30 & 3.20 & 0.74 & 7.52 & 1.43 \\
10000 & 1.12 & 0.33 & 4.19 & 0.83 & 10.64 & 1.60 \\
\hline
\end{tabular}




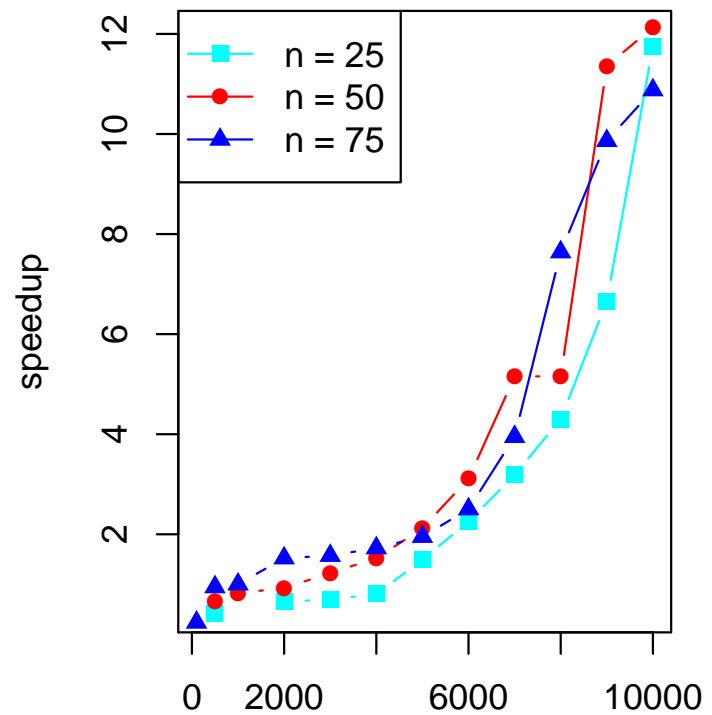

$\mathrm{p}$

(a) neighbor searching only

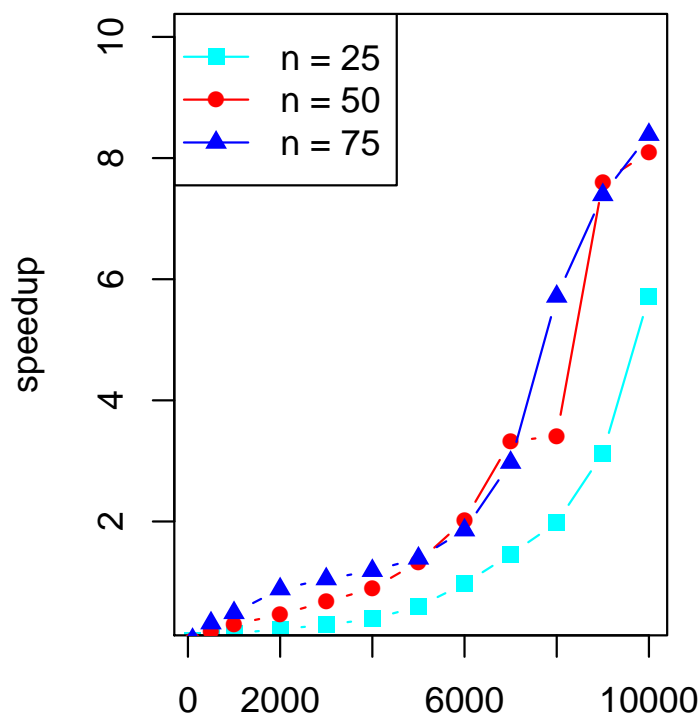

$\mathrm{p}$

(b) overall speedup

Figure 2.4: Random KNN speedup for simulated data, $m=\lfloor\sqrt{p}\rfloor$

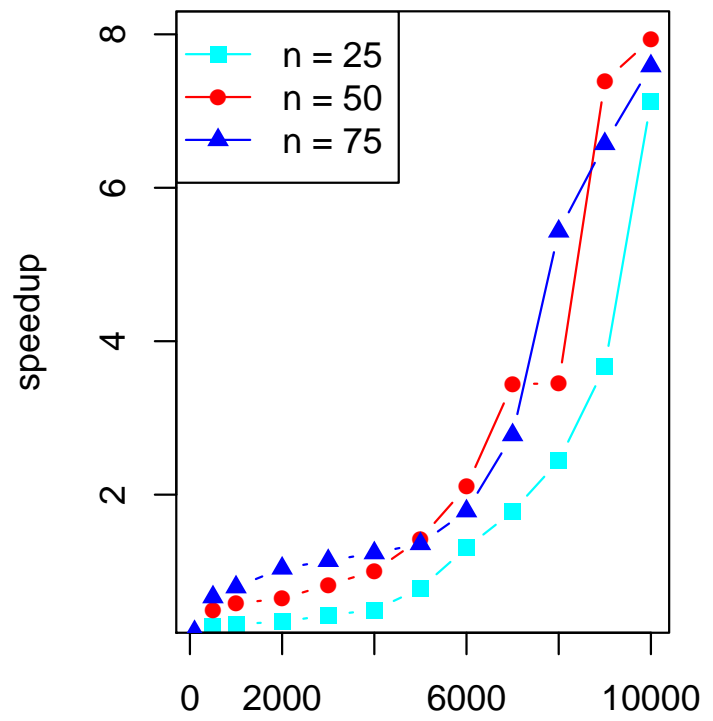

$p$

(a) neighbor searching only

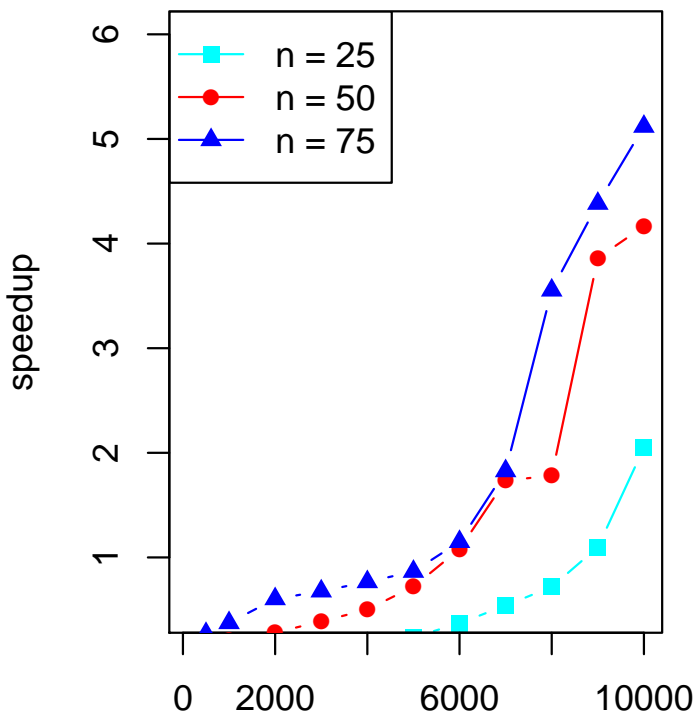

$\mathrm{p}$

(b) overall speedup

Figure 2.5: Random KNN speedup for simulated data, $m=\left\lfloor\log _{2}(p)\right\rfloor$ 


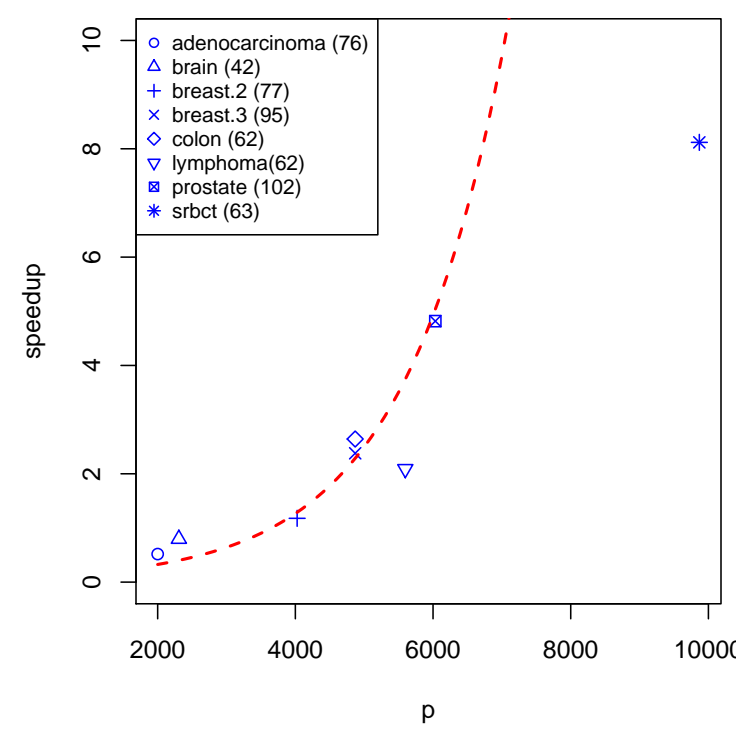

(a) neighbor searching only

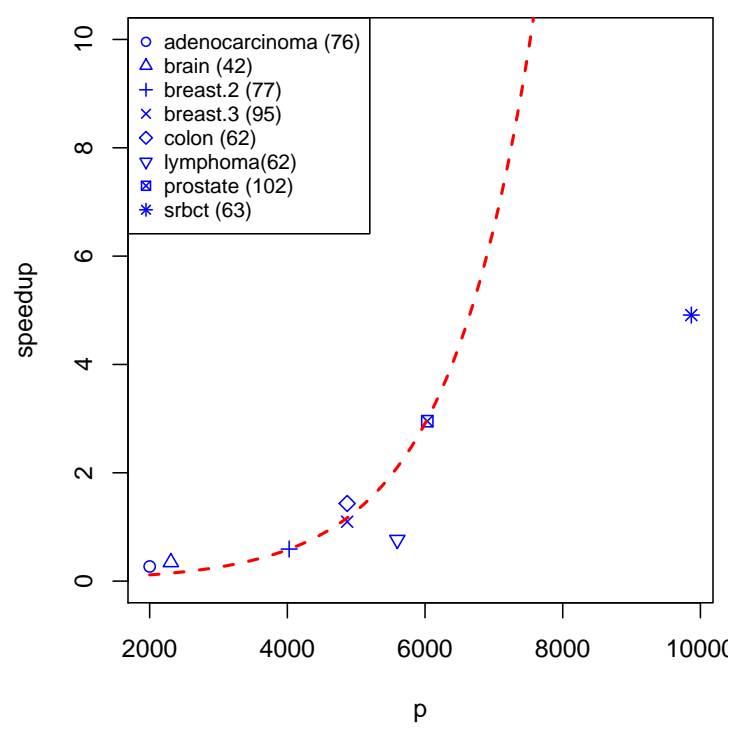

(b) overall speedup

Figure 2.6: Random KNN speedup for microarray data, $m=\lfloor\sqrt{p}\rfloor$

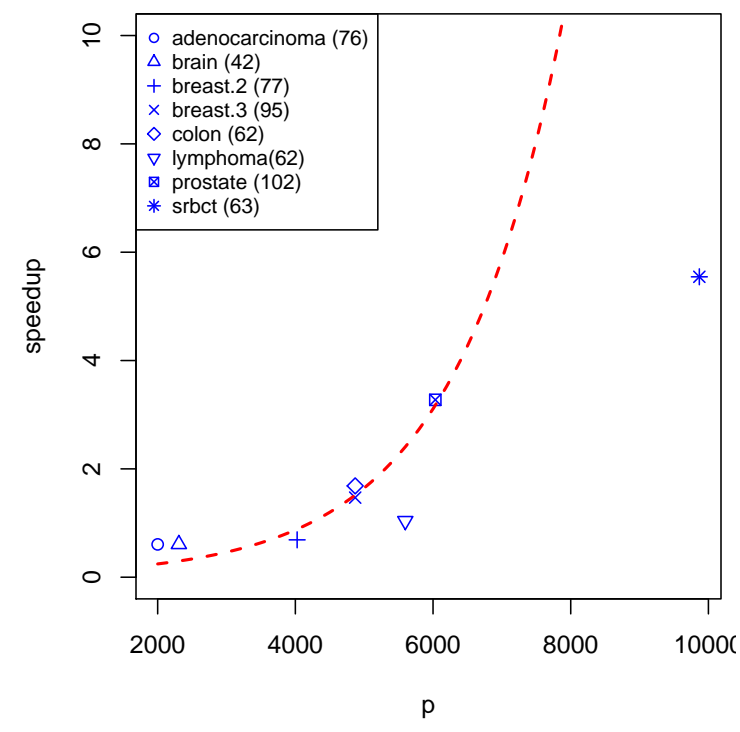

(a) neighbor searching only

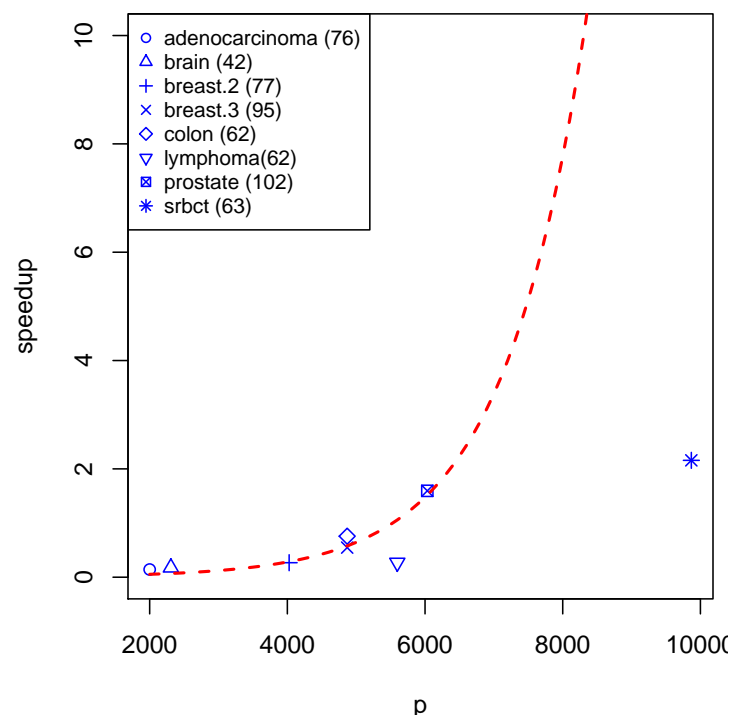

(b) overall speedup

Figure 2.7: Random KNN speedup for microarray data, $m=\left\lfloor\log _{2}(p)\right\rfloor$

In terms of computational complexity, our Random KNN approach obviously partially tackled one aspect of the curse of dimensionality problem. The exponential 
growth of the running time with number of features is eased. This enables Random KNN to model large scale applications with large $p$ and/or large $n$, especially for genome-scale data. Since each base KNN can be run independently, the Random KNN method can be readily parallelized on a machine with multiple computational units such as Beowulf cluster computer: $\rrbracket^{円}$ or multicore processor systems.

\subsection{Parameter Setting}

Random KNN has three tuning parameters: the number of nearest neighbors, $k$; the number of random KNN's, $r$; and the number of features for each base KNN, $m$. In this section, we will discuss these parameters and the choices for them.

\subsubsection{Number of nearest neighbors, $k$}

The choice of $k$ for KNN has been studied in the literature. But the problem is still not well solved. The general guideline proposed by Fix and Hodges ${ }^{422}$ is the two-condition rule:

$$
\lim _{n \rightarrow \infty} k=\infty
$$

and

$$
\lim _{n \rightarrow \infty} k / n=0 .
$$

Devroye $(1982)^{36}$ suggested that:

$$
\lim _{n \rightarrow \infty} k / \log \log n=\infty
$$

\footnotetext{
1 http://www . beowulf .org
} 
and

$$
\lim _{n \rightarrow \infty} k / n=0
$$

And Devroye et al. (1994) ${ }^{37}$ suggested that:

$$
\lim _{n \rightarrow \infty} k / \log n=\infty
$$

and

$$
\lim _{n \rightarrow \infty} k / n=0
$$

$k \propto \sqrt{n}$ is also widely recommended. These conditions imply that $k$ should be large but much smaller than $n$. These recommendations are for large samples (asymptotic behavior); it is difficult to make statements for finite-sample applications. We conducted a stimulation study for different distributions when KNN is used to estimate entropy ${ }^{84}$. It is difficult to determine a functional form between $k$ and $n$, and the choice of $k$ is also distribution-dependent. Hall et al. ${ }^{[58}$ used a bootstrapping method to get an optimum $k$, which minimizes the mean error rate and then correct this $k$ by the resampling proportion and the data dimensionality.

Essentially, the problem of choosing of $k$ is still not well solved in theory. In practice, since the sample sizes are often relatively small, $k$ is also usually small. Crossvalidation is therefore widely adopted. For Random KNN, the theoretical side of this problem is more difficult. Choosing $k$ by experimentation is a realistic approach. We applied Random KNN to 8 cancer gene expression data sets with different $k$ and $r$. Relations of performance versus $k$ are plotted on Figure 2.8. From this figure, Random KNN is rather robust for different $k$ values. The best $k$ 's for these data sets are from 1 to 9 . So in practice, the appropriate value of $k$ can be easily determined by several trials for a given application. 

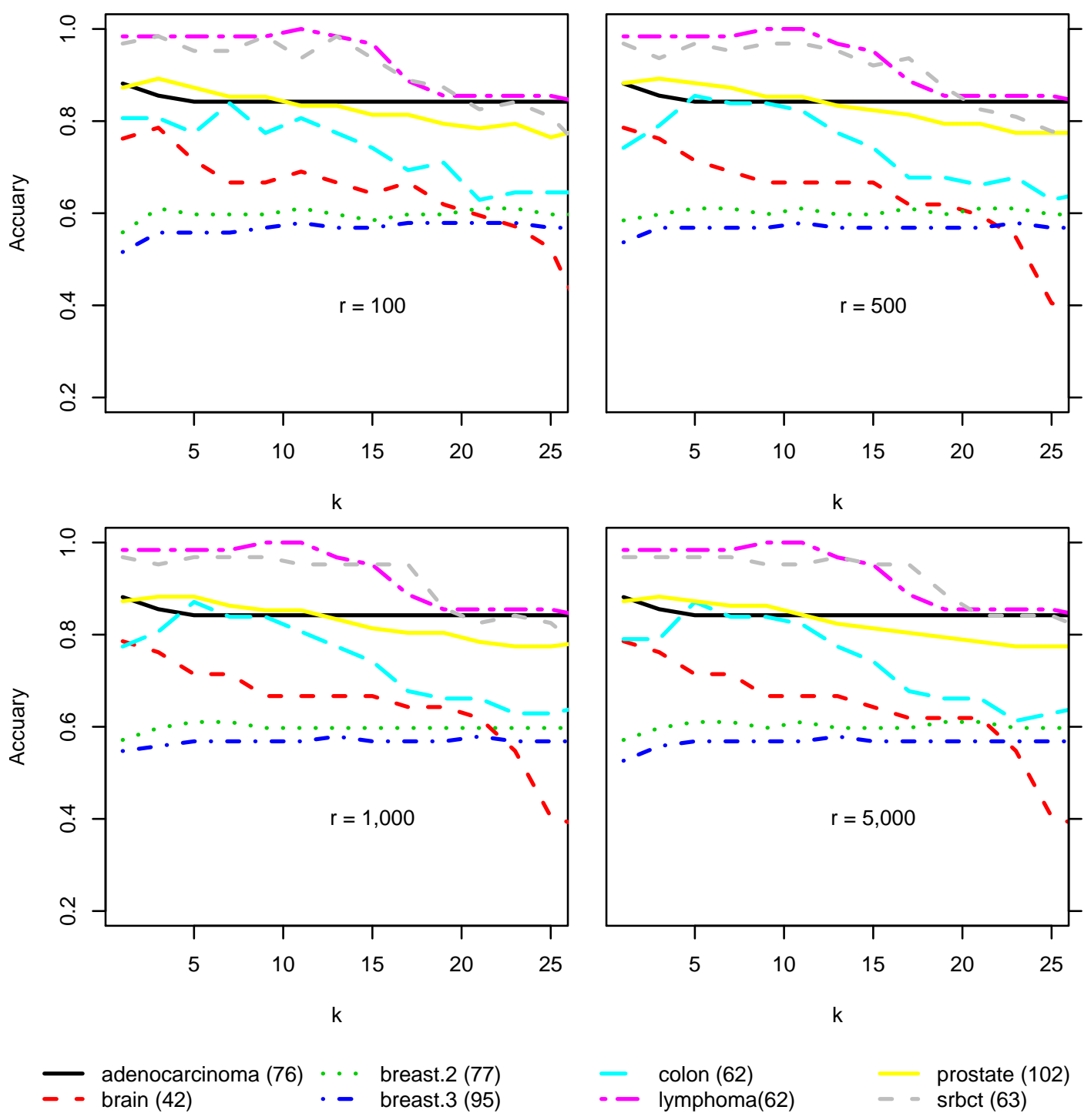

Figure 2.8: Random KNN accuracies for different number of nearest neighbors, $k$

\subsubsection{Number of features, $m$}

The number of features for each random KNN, $m$, is another important parameter of the Random KNN procedure. To generate the maximum number of different feature subsets, we need to have $m=\frac{p}{2}$. Essentially, this means that $r \leq\left(\begin{array}{c}p \\ m\end{array}\right)=\left(\begin{array}{c}p \\ p / 2\end{array}\right)$. On the other hand, to ensure diversity in these feature subsets (i.e., for less redundancy 
or overlap among these subsets), $m$ needs to be small. For any two subsets of $m$ features, the number of common features between them, $Y$, is a hypergeometric random variable. Imagine the $m$ features in set 1 are white and the remaining $p-m$ features are black. Then randomly draw $m$ features from these $m$ white and $p-m$ black features without replacement. The number of white features drawn is $Y$. This is the classical hypergeometric model and its probability mass function is:

$$
P(Y=y)=\frac{\left(\begin{array}{c}
m \\
y
\end{array}\right)\left(\begin{array}{c}
p-m \\
m-y
\end{array}\right)}{\left(\begin{array}{c}
p \\
m
\end{array}\right)}
$$

Therefore, $\mathbb{E}(Y)=m \frac{m}{p}=\frac{m^{2}}{p}$. If we let $m \leq \sqrt{p}$, then $E(Y) \leq 1$.

Smaller values of $m$ are also important in considering the computational time required for the nearest neighbor searching. Therefore, $m$ should be less than $\frac{p}{2}$. We tested Random KNN with different $m$, as shown in Figure 2.9. Sample sizes and numbers of features are given in the inset. From this test, most data sets perform well with $m=\sqrt{p}$. So taking $m=\sqrt{p}$ is a reasonable choice.

\subsubsection{Number of random KNN's, $r$}

The number of random KNN's, $r$, may be chosen according to the equations 2.12, 2.13 and 2.14. We tested different values of $r$ on the 8 tumor microarray data sets as shown in Figure 2.10. For each data set and for a given $r$, Random KNN were repeated 30 times and its performance was averaged. This figure reveals the nondecreasing property of the accuracy of Random KNN with respect to $r$. To guarantee convergence, a large $r$ can be used. But for prudence, a proper size of $r$ may be chosen. For these data sets, the accuracies converge after $r>100$ when $m=\sqrt{p}$. When $m=\log _{2}(p), r$ needs to larger and if $m=\log _{2}^{2}(p), r$ can be smaller. The number of KNN's has a larger impact on prostate, srbct (small round blue cell tumors), brain 


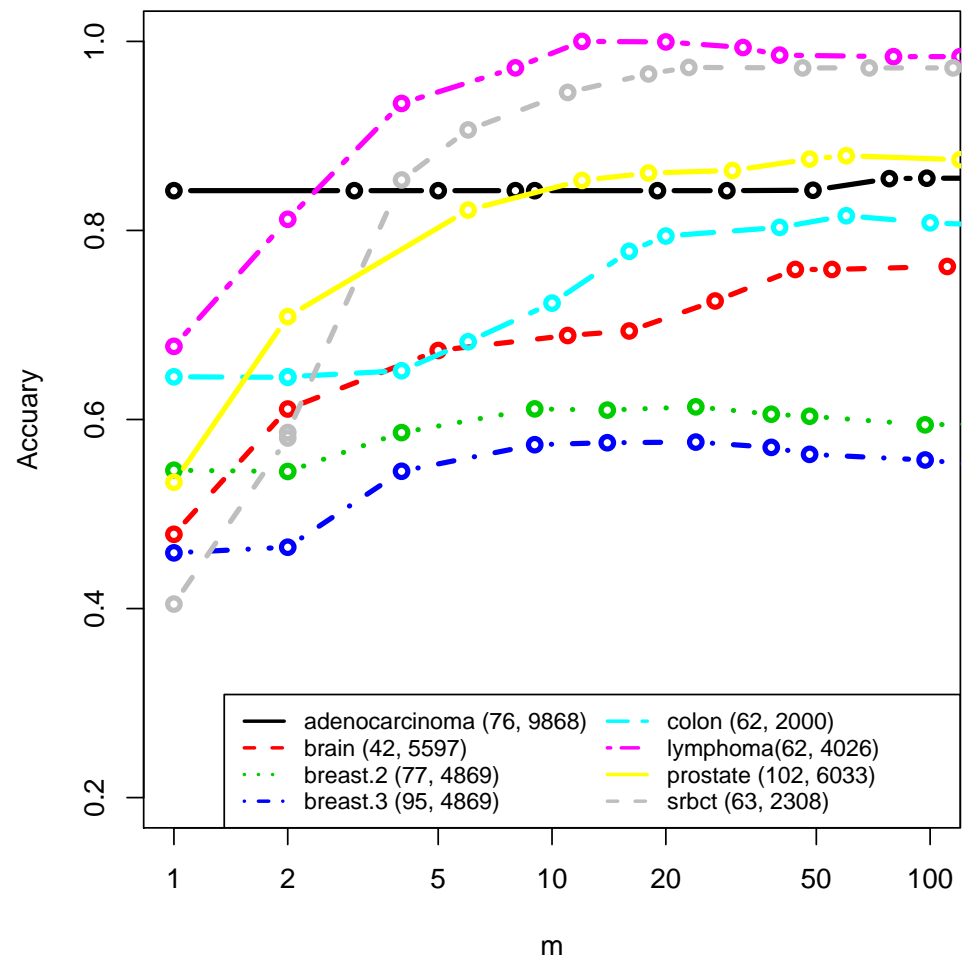

Figure 2.9: Random KNN accuracies for different feature subset size, $m(r=500, k=$ 3,30 runs)

and lymphoma data sets than others. For other data sets, the convergence is faster and need a smaller $r$. The behavior of the different $r$ values is quite data-dependent. Fast convergence may also indicate that most of the features are similar and thus redundant. For microarray data, usually a small number of genes are the key genes; thus, feature selection is necessary.

In summary, although we have three moving parts for Random KNN, they can be readily set. We recommend to set $m=\lfloor\sqrt{p}\rfloor, r=200-500$, and $k$ between 1 and 15 for microarray data applications. 

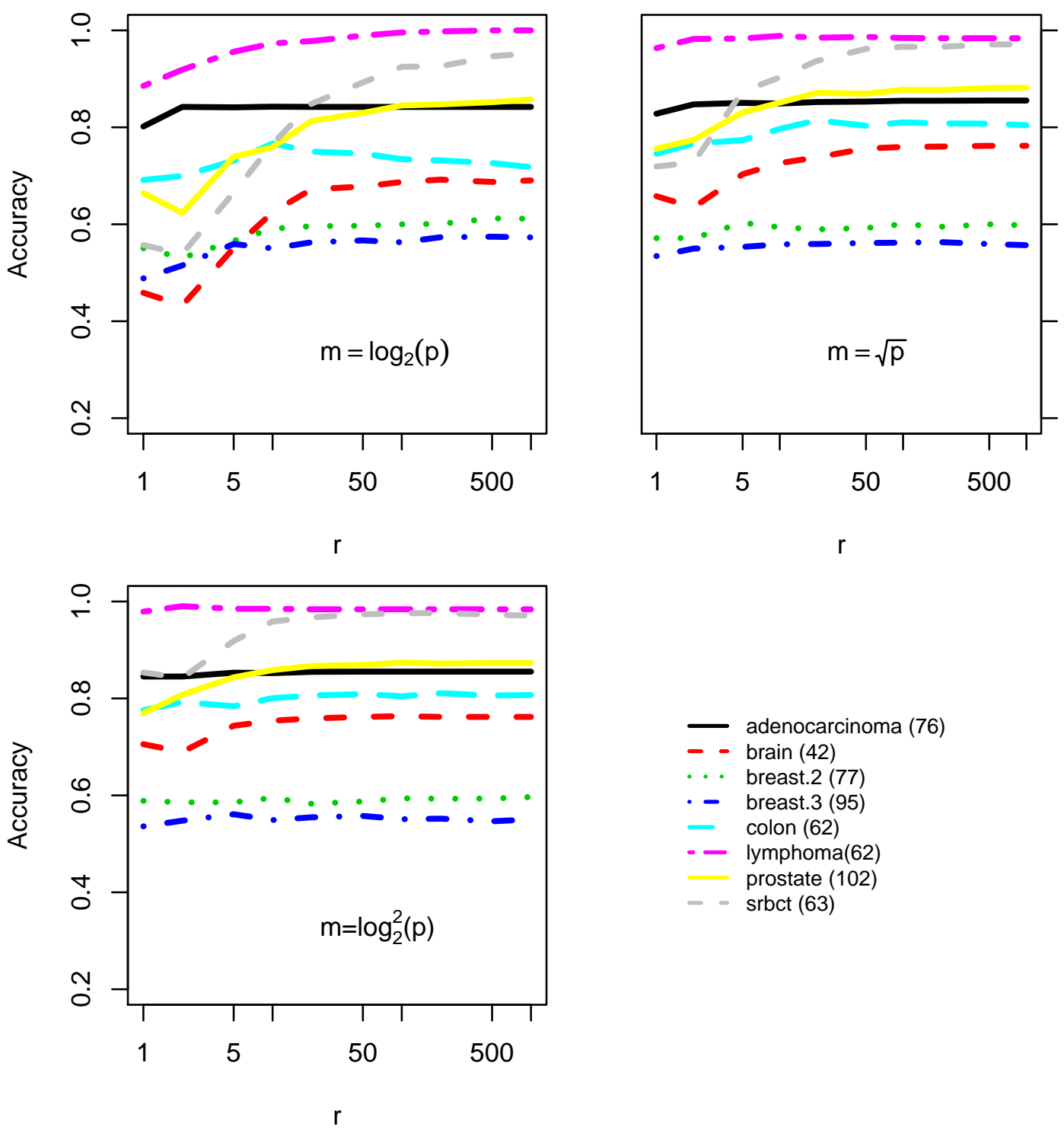

Figure 2.10: Random KNN accuracies for different number of KNN's, $r$ 


\subsection{Cancer Gene Expression Data Sets}

In this study, microarray data sets for human cancer will be extensively used. In Table 2.3, 8 microarray data sets are listed. These data sets are publicly available ${ }^{34}$. In the table, SRBCT stands for Small Round Blue Cell Tumor.

Table 2.3: Cancer microarray data sets

\begin{tabular}{l|c|c|l|c}
\hline Data Set & $\begin{array}{c}\text { No. of } \\
\text { classes, } c\end{array}$ & $\begin{array}{c}\text { Sample Size } \\
n\end{array}$ & $\begin{array}{l}\text { Class Sample } \\
\text { Size, } n_{j}\end{array}$ & $\begin{array}{c}\text { No. of } \\
\text { Genes, } p\end{array}$ \\
\hline \hline Adenocarcinoma & 2 & 76 & 64,12 & 9868 \\
Brain & 5 & 42 & $10,10,10,4,8$ & 5597 \\
Breast (2 classes) & 2 & 77 & 33,44 & 4869 \\
Breast (3 classes) & 3 & 95 & $33,44,18$ & 4869 \\
Colon & 2 & 62 & 22,40 & 2000 \\
Leukaemia I & 2 & 38 & 27,11 & 3051 \\
Leukaemia II & 2 & 34 & 22,14 & 3051 \\
Lymphoma & 3 & 62 & $42,9,11$ & 4026 \\
Prostate & 2 & 102 & 50,52 & 6033 \\
SRBCT & 4 & 63 & $23,20,12,8$ & 2308 \\
\hline
\end{tabular}

The following is the description $\underline{33}$ of the data sets:

The data sets Colon, Prostate, Lymphoma, SRBCT and Brain were obtained, as binary R files, from Marcel Dettling's web site http://stat.

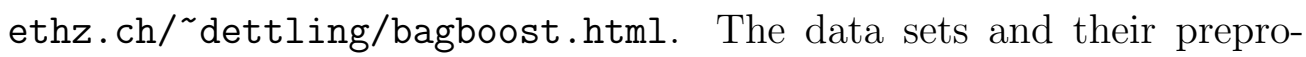
cessing are fully described in Dettling \& Bühlmann (2002)

The adenocarcinoma data set is from Ramaswamy et al. (2003) ${ }^{96}$. We used the data from the 12 metastatic tumors and 64 primary tumors. The original data set included 16063 genes from Affymetrix chips. The data (DatasetA_Tum_vsMet.res), downloaded from http://www-genome.wi. mit.edu/cgi-bin/cancer/, had already been rescaled by the authors. We took the subset of 9376 genes according to UniGene mapping, thresholded data, and filtered by variation as explained by the authors. The final 
data set contains 9868 clones (several genes were represented by more than one clone); of these, 196 had constant values over all individuals.

The breast cancer data set is from van 't Veer et al. (2002) ${ }^{115}$. The data were downloaded from http://www.rii.com/publications/2002/ vantveer.htm (we used the files ArrayData_less_than_5yr.zip, ArrayData_greater_than_5yr.zip, ArrayData_BRCA1.zip, corresponding to 34 patients that developed distant metastases within 5 years, 44 that remained disease-free for over 5 years, and 18 with BRCA1 germline mutations and 2 with BRCA2 mutations). As done by the authors, we selected only the genes that were "significantly regulated" (see their definition in the paper and supplementary material), which resulted in a total of 4869 clones. Because of the small sample size, we excluded the 2 patients with the BRCA2 mutation. We used 5-nearest neighbor imputation for the missing data, as for the NCI 60 data set. Finally, we excluded from the analyses the 10th subject from the set that developed metastases in less than 5 years (sample 54, IRI000045837, in the original data files), because it had 10896 missing values out of the original 24481 clones, and was an outstanding outlying point both before and after imputation. The breast cancer data set was used both for two class comparison (those that developed metastases within 5 years vs. those that remain metastases free after 5 years) and for three group comparisons.

The Leukemia data sets are those known as Golub's microarray data ${ }^{[50}$, which are widely cited. The data are from two types of leukemia: ALL(Acute Lymphocytic Leukemia) and AML (Acute Myeloid Leukemia). The full data sets can be downloaded $^{\underline{49}}$ from the Internet. These two data sets are also available in golubE- 
sets package of $\mathrm{R}^{109}$ as training and testing sets. There are 38 mRNA samples in the set I (training set) and 34 in the set II (testing set). The microarray platform is Affymetrix ${ }^{\circledR}$ HGU6800 that contains 7,129 probe sets (features). After applying Golub's preprocessing steps, $p=3051$ probe sets are left. The expression values are then normalized to mean 0 and standard deviation 1 for each sample. Set $\mathrm{I}$ is also available in multtest package.

\subsection{Application of Random KNN to Leukemia Mi- croarray Data}

Here, we will apply Random KNN classifier to the leukemia data sets. We use the set I as the training set and set II as the testing set. We choose $m=\lfloor\sqrt{p}\rfloor=55$. To choose $r$, we set $\eta_{b}=0.999$. Thus $r=821$ and $\nu=14.8$. The following is the output:

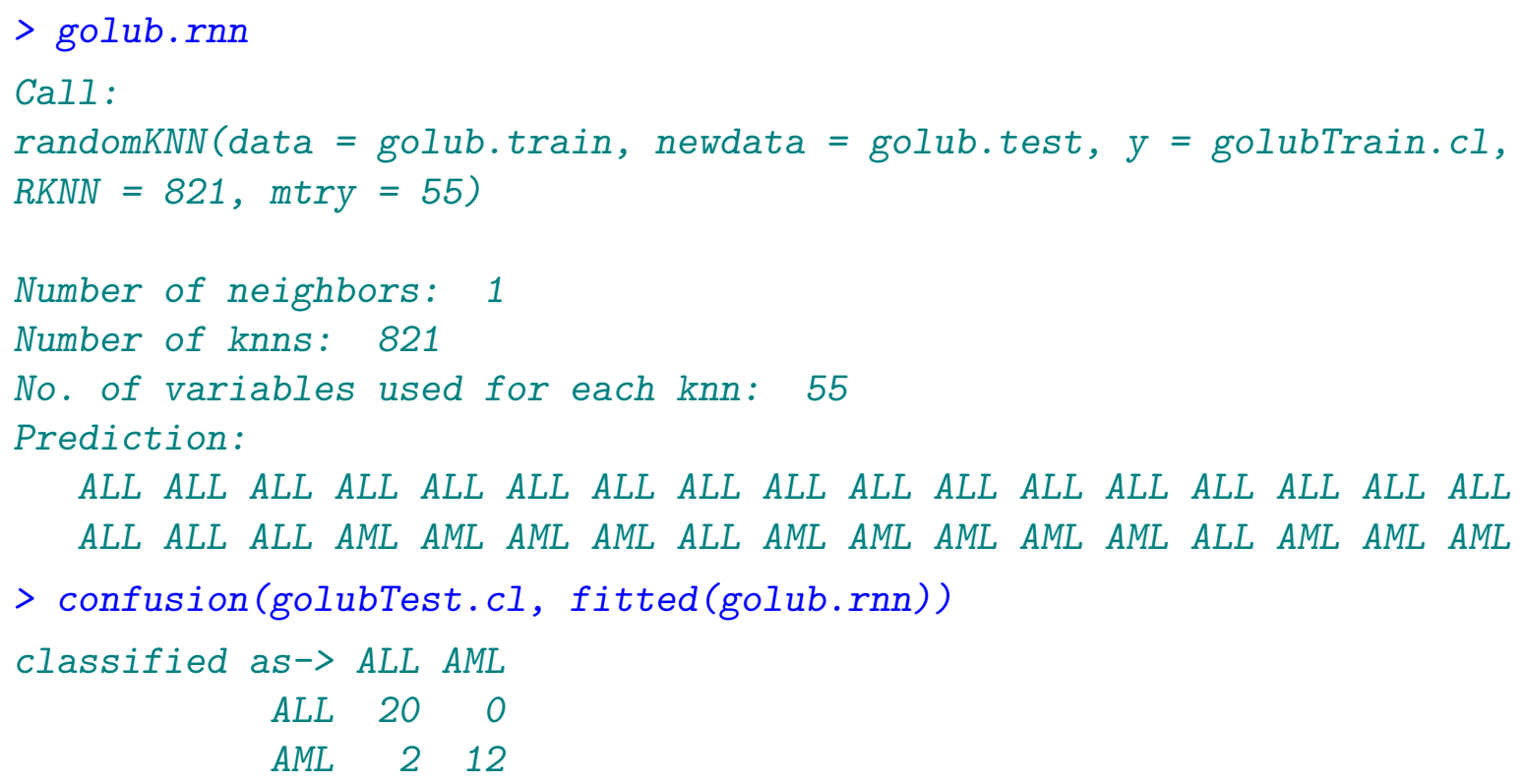

The confusion matrix will be used to show the number of cases correctly or wrongly classified by a classifier. Row labels are the observed or experimental classes and 
column labels are the classifications by the classifier. For a perfect classification, the confusion matrix will be diagonal, otherwise off-diagonal cells are the number of errors.

The confusion matrix of above random KNN classifier shows that of the 34 test samples, only two AML are misclassified as ALL. Golub obtained 100\% accuracy while Dudoit et al. found 3 cases were difficult to classify 4 ․․․

In above example, every feature is used and the multiplicities are between 4 and 34 as shown by following output:

$>\operatorname{length}(\operatorname{varNotUsed}($ golub.rnn))

[1] 0

$>$ golub.varUsed <- varUsed(golub.rnn)

$>$ summary (golub.varUsed)

$\begin{array}{rrrrrr}\text { Min. 1st Qu. } & \text { Median } & \text { Mean } 3 r d \text { Qu. } & \text { Max. } \\ 4.0 & 12.0 & 15.0 & 14.8 & 17.0 & 34.0\end{array}$

To check the distribution of feature multiplicities in these random KNNs, a histogram is plotted in Figure 2.11.

The multiplicity is approximately symmetric around 15 .

If we set $\eta_{b}=0.9$, then $r=565$ and $\nu=10.2$.

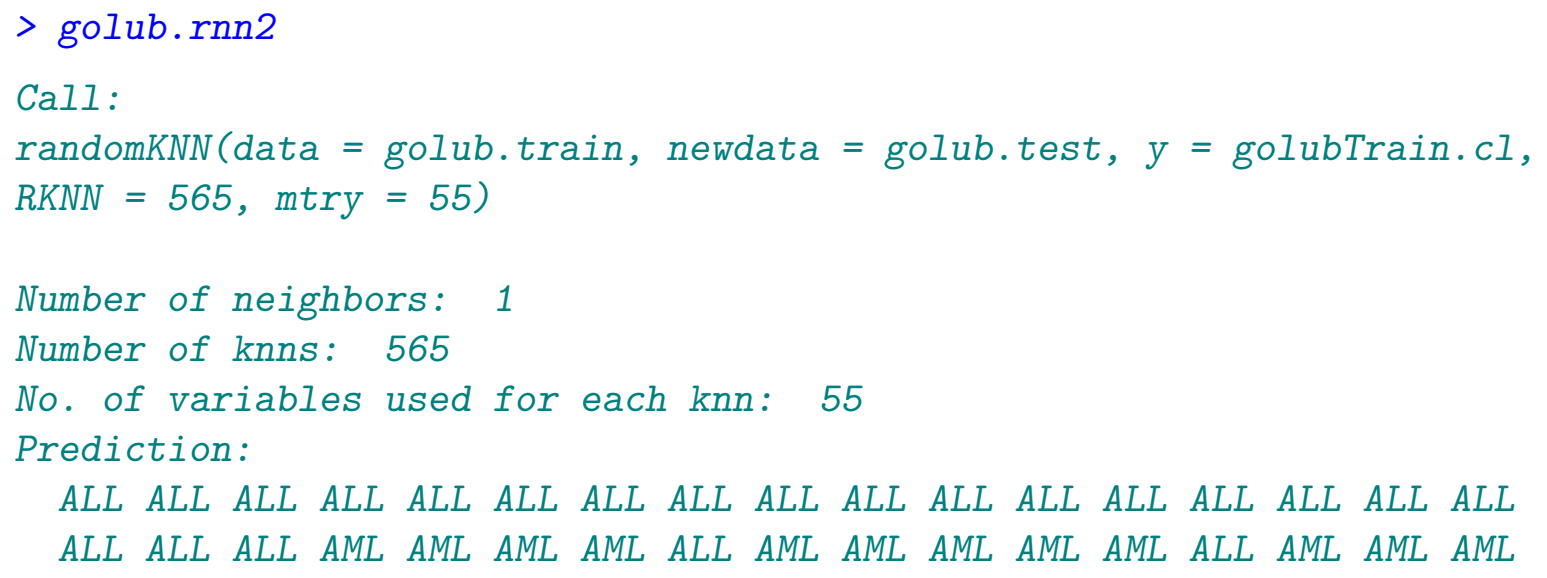




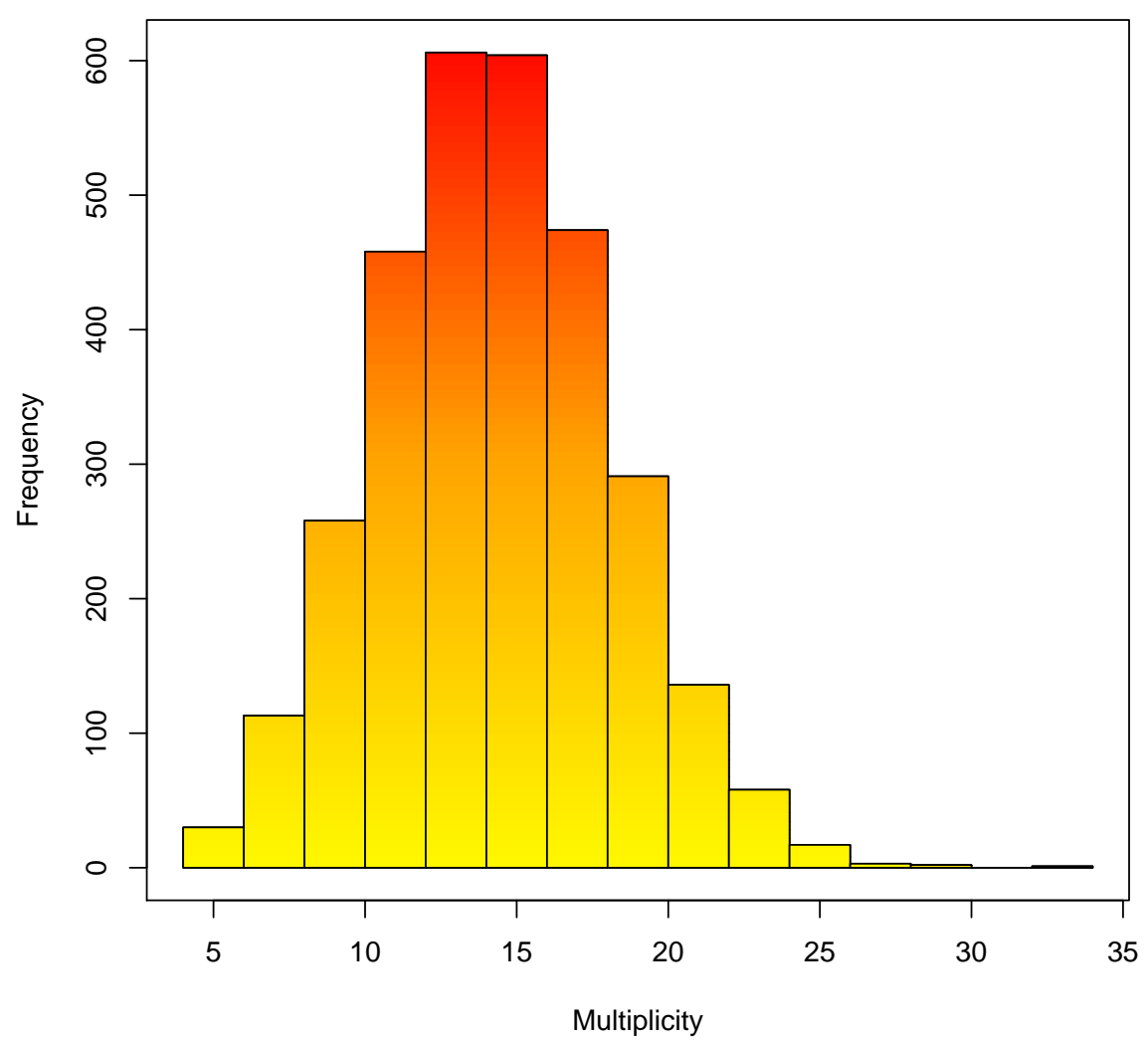

Figure 2.11: Histogram of the feature multiplicities

Every feature is used at least once and at most 22 times as shown by following output.

$>$ length (varNotUsed (golub.rnn2))

[1] 0

$>$ golub.varUsed2 <- varUsed (golub.rnn2)

$>$ summary (golub.varUsed2)

Min. 1st Qu. Median Mean 3rd Qu. Max.

$\begin{array}{llllll}1.00 & 8.00 & 10.00 & 10.19 & 12.00 & 22.00\end{array}$

The confusion matrix is as follows:

> confusion(golubTest.cl, fitted(golub.rnn2)) 


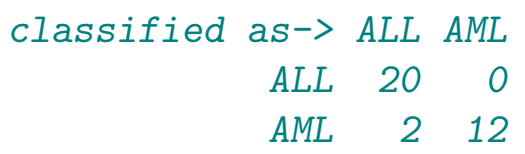

The results show that two AML cases are classified ALL. Although the classification is quite good, we prefer a simpler model that uses a few genes, i.e., a gene signature. The next chapter will address the feature selection problem. 


\section{Chapter 3}

\section{Feature Selection with Random KNN}

For high dimensional data, especially for microarray data, most of the features are not relevant to the target variable. Removing redundant features is beneficial as discussed in chapter 1 . In this chapter, feature selection using Random KNN will be developed.

\subsection{Bidirectional Voting and Variable Ranking}

In order to select a subset of variables that have classification capability, the key problem is to define some criteria to rank the variables. We define a measure, called support. Each feature $f$ will appear in some KNN classifiers, say set $\mathbf{C}(f)$ of size $M$, where $M$ is the multiplicity as defined in chapter 2 . In turn, each classifier $c \in \mathbf{C}(f)$ is an evaluator of its $m$ features, say, set $\mathbf{F}(c)$. For instance, we can take its accuracy as a performance measure for those features. The mean accuracy of these KNN classifiers (support) is a measure of the feature relevance with the outcome. Thus we have a ranking of the features. We call this scheme bidirectional voting. Each feature 
randomly participates in a series of KNNs to vote a case for classification, and in turn, each case casts a vote for each participating feature. The pseudocode of the algorithm is listed in Algorithm 3.1. A schematic diagram of the bidirectional voting procedure is shown in Figure 3.1 .

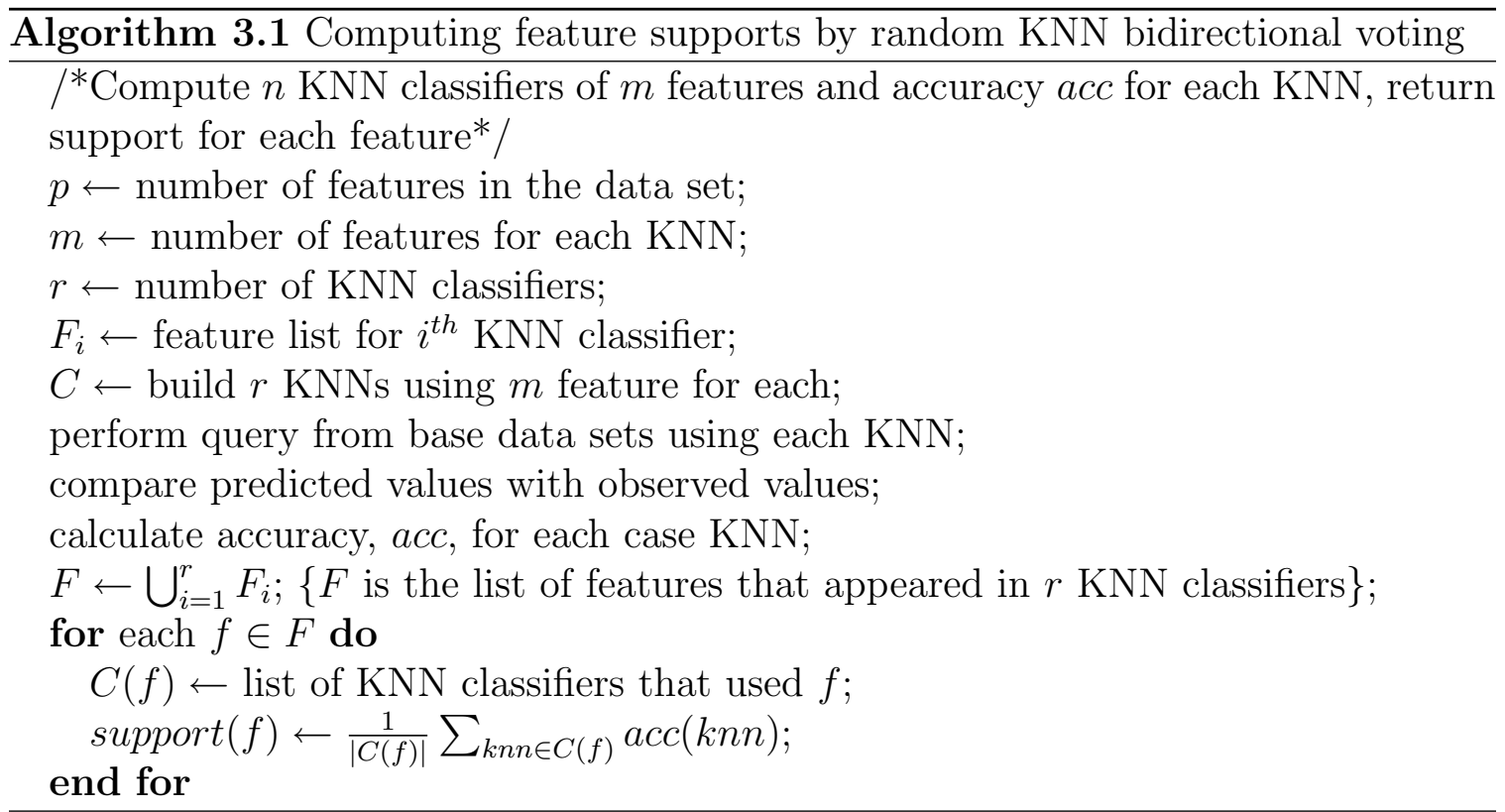

To compute feature supports, data is partitioned into base and query subsets. Two partition methods may be used: (i) dynamic partition; (For each KNN, the cases are randomly partitioned. One half is the base subset and the other half is the query subset.) (ii) fixed partition. The data set is partitioned once, and for all KNNs, the same base subset and query subset are used. That is, all base subsets are the same and all query subsets are also the same. In a sense, support is similar to Random Forest's importance measure. The higher the supports, the more relevant the features.

\subsubsection{Complexity of Computing Feature Support}

For each KNN, we have the time complexity as follows: 


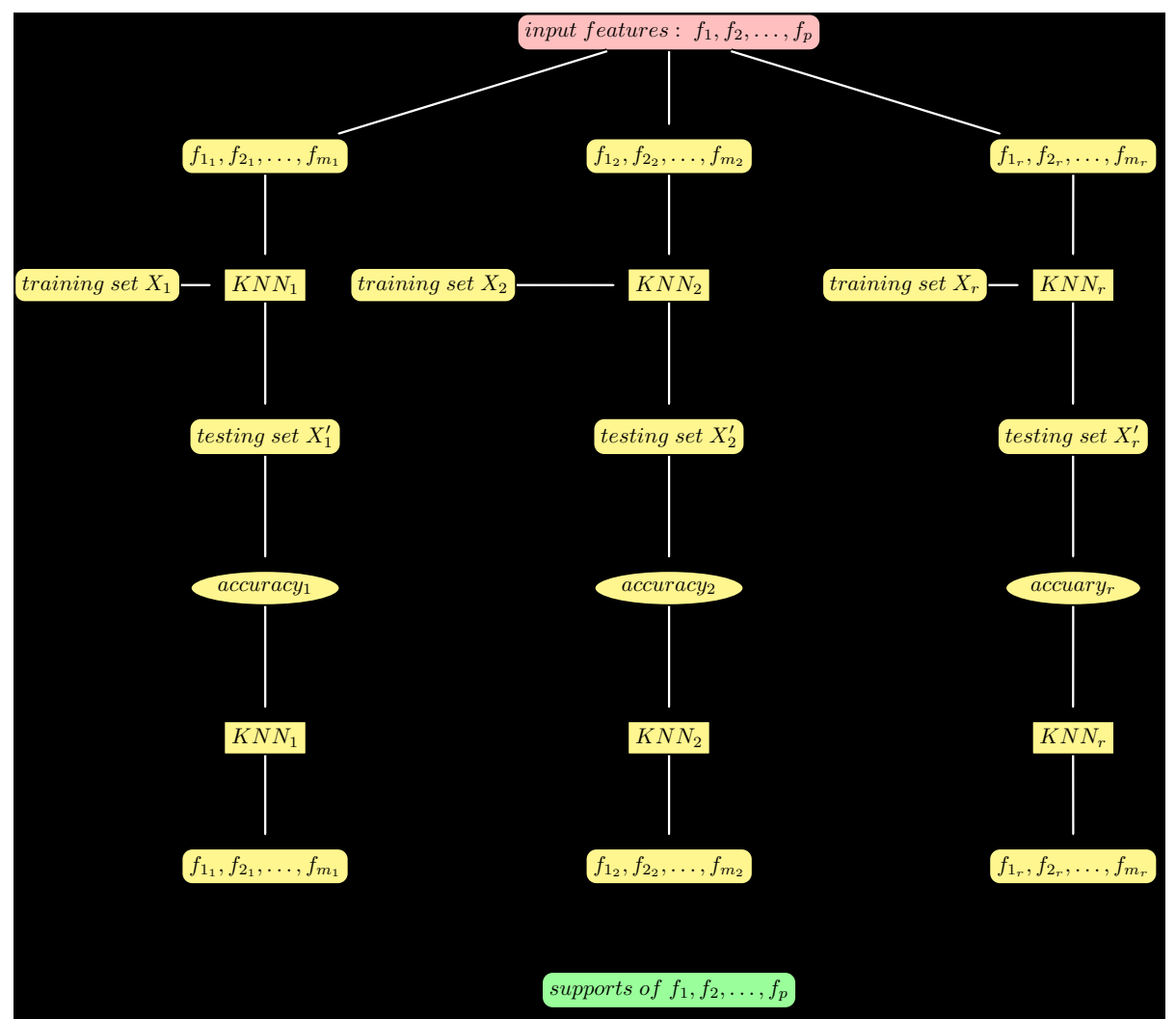

Figure 3.1: Diagram of bidirectional voting of random KNN

- Data Partition: $O(n)$;

- Near Neighbor Searching: $O\left(k 2^{m} n \log n\right)$;

- Classification: $O(k n)$;

- Computing accuracy: $O(n)$;

Adding the above 4 items together, we get a subtotal time for one $\mathrm{KNN}: O\left(k 2^{m} n \log n\right)$.

For Random KNN, we have $r$ KNN's; thus the total time for the above steps is $O\left(r k 2^{m} n \log n\right) . r m$ features are used in the Random KNN, the time for computing supports from these accuracies is $O(r m)$. Thus the overall time is $O\left(r k 2^{m} n \log n\right)+$ $O(r m)=O\left(r\left(m+k 2^{m} n \log n\right)\right)=O\left(r k 2^{m} n \log n\right)$. 


\subsubsection{Example}

The following is the output for Golub's 38 leukemia training samples using dynamic partitions, where the accuracy and the confusion matrix provide overall performance measures for the collection of KNN's.

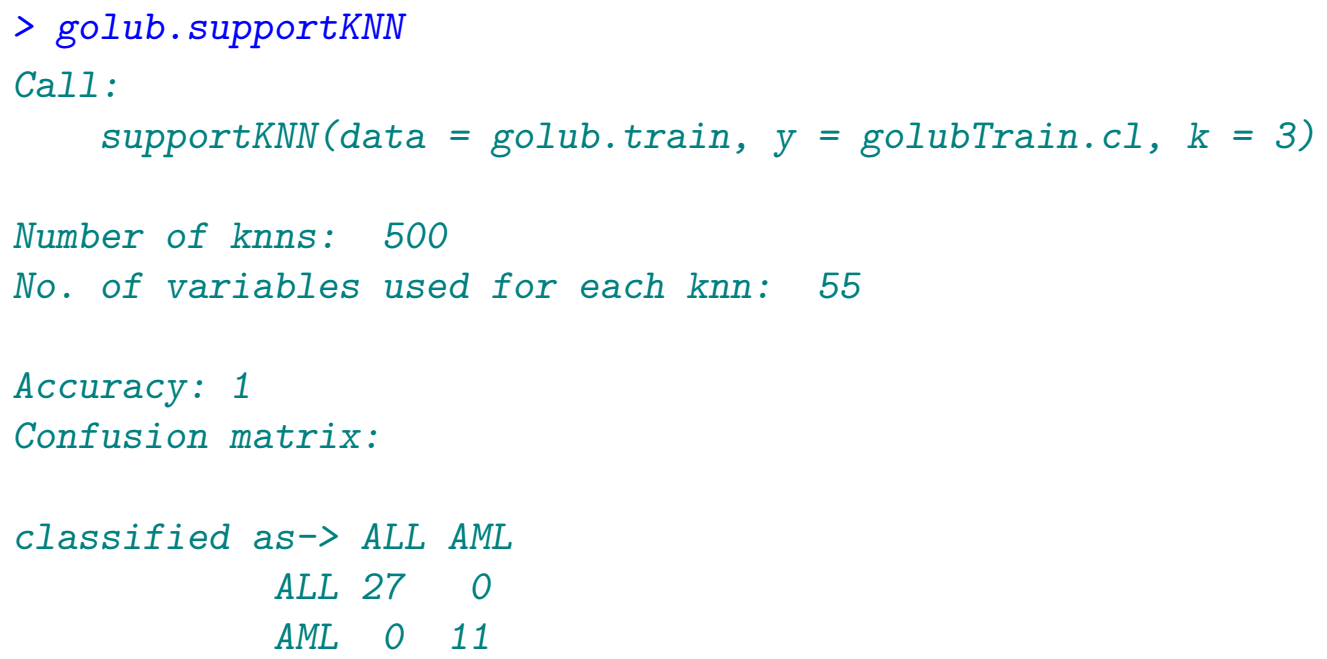

The fixed partition method is also applied to this data set for support calculation:

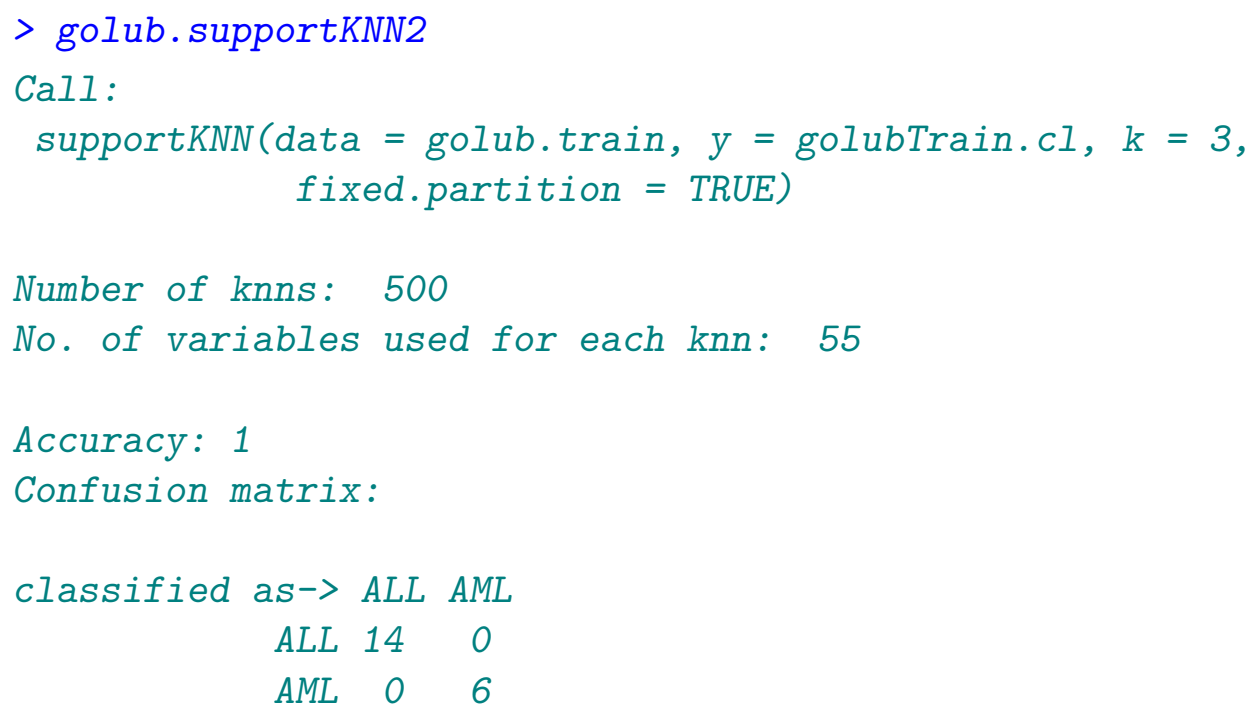

Figure 3.2 displays the 30 most relevant genes by the support criterion for this data set using fixed and dynamic partitions. 

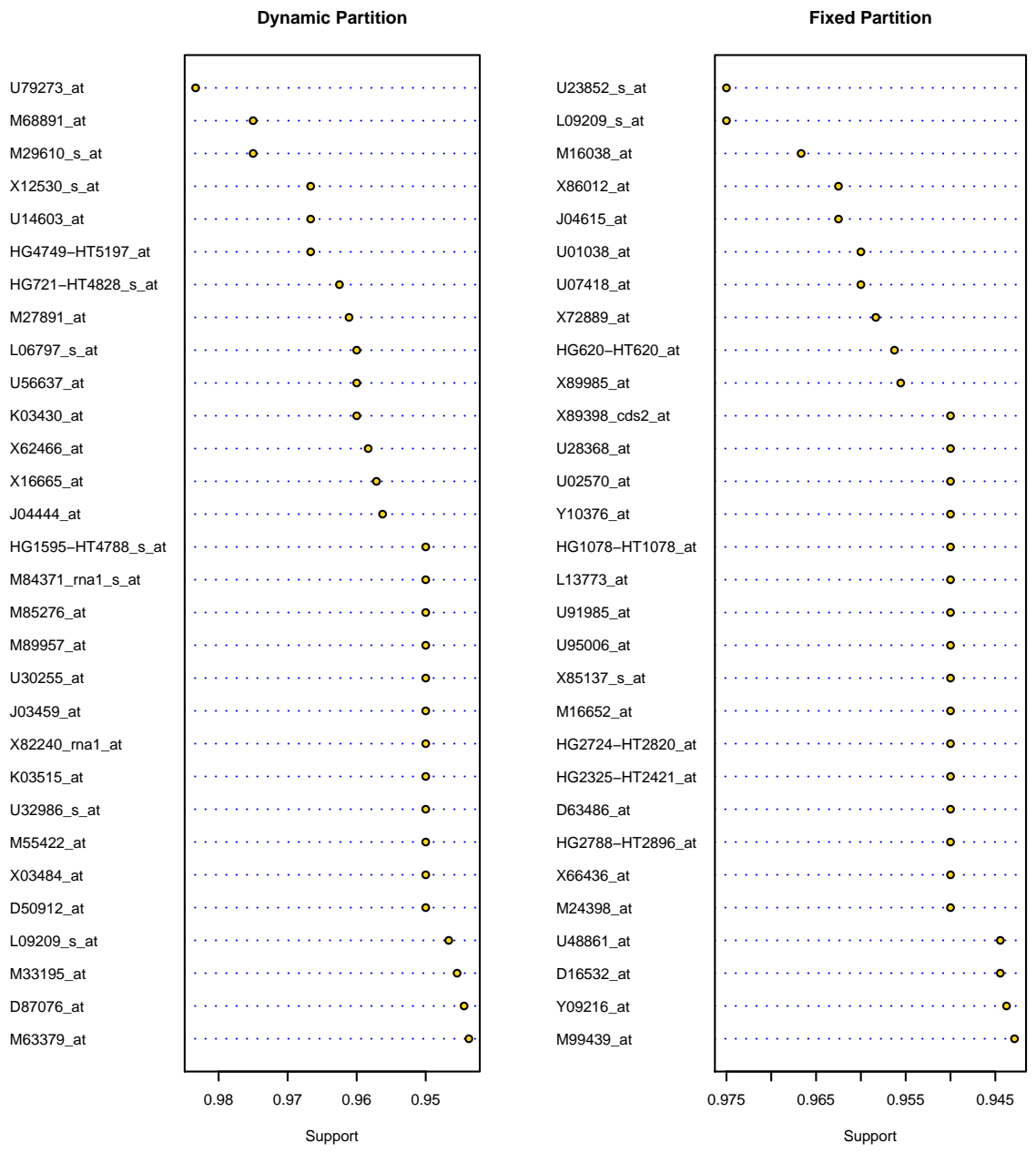

Figure 3.2: Supports of the first 30 genes of the Golub leukemia data

\subsection{Feature Selection with Random KNN}

With feature supports, we can directly select high rank features after running the support algorithm on the entire data set. We call this direct selection. But this simple approach may be too aggressive and risky for high dimensional data. We take a more conservative and safer approach, namely, multiple rounds of screening. That is, we recursively apply the direct selection procedure. To balance between speed and classification performance, we split recursion into two stages. First stage 
is fast, and the number of variables is reduced by a given ratio ( $1 / 2$ by default). This stage is a geometric elimination process since the dimension to be kept is a geometric progression. In the second stage, a fixed number of features (one by default) are dropped each time. This is a linear reduction process. Finally, a relatively small set of variables will be selected for the final models. To aid in this recursive procedure, another assessment criterion for a set of features is required. We use the average accuracy of the $r$ random KNNs (alternatively, mean support could be used). After the first stage, we can plot the average accuracies against the number of features. The iteration just before the maximum accuracy is reached is called pre-max iteration. We use the data set used in pre-max iteration for the second stage selection. The algorithm is shown in Algorithm 3.2 (page 47).

\subsubsection{Complexity}

As discussed in the last section, the time to compute feature supports is $O\left(r k 2^{m} n \log n\right)$, and sorting these supports will take $O(p \log p)$. Since for most applications, $\log p<$ $n \log n$, and $p<r k 2^{m}$, the total time for calculating and ranking feature supports is $O\left(r k 2^{m} n \log n\right)$.

In stage-one, the number of features decreases geometrically with proportion $q$. For simplicity, let us take $m$ be the square-root of $p$ and keep $r$ fixed. Thus the sum of the component $2^{m}$ is $2^{\sqrt{p}}+2^{\sqrt{p q}}+2^{\sqrt{p q^{2}}}+2^{\sqrt{p q^{3}}}+2^{\sqrt{p q^{4}}}+\ldots$. The first term is dominant, since $q$ is a fraction. Thus the total time is $O\left(r k 2^{\sqrt{p}} n \log n\right)$

In stage-two, each time a fixed number of features will be removed. In the extreme case, only one feature is removed per iteration, the total time will be $O\left(r k 2^{p_{1}+1} n \log n\right)$, where $p_{1}$ is the number of features at the start of stage-two, and usually $p_{1}<p^{1 / 2}$. So on average, $O\left(r k 2^{p_{1}+1} n \log n\right)=O\left(r k 2^{\sqrt{p}} n \log n\right)$. 


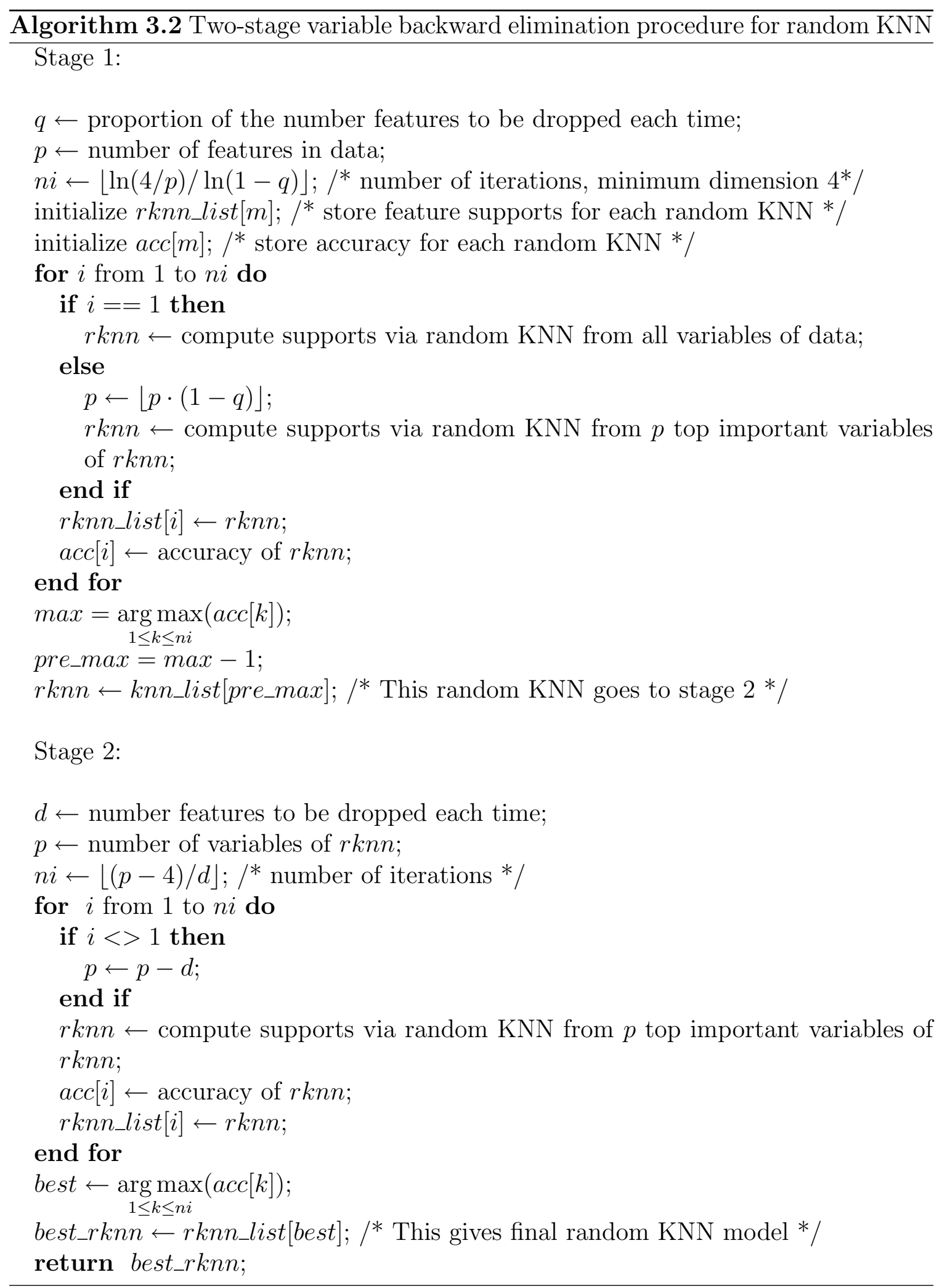


Therefore, the total time for entire algorithm is $O\left(r k 2^{\sqrt{p}} n \log n\right)$, same as that for using Random KNN for classification, at $m=\sqrt{p}$. Essentially, in theory, feature selection does not degrade the complexity of Random KNN.

\subsubsection{Example}

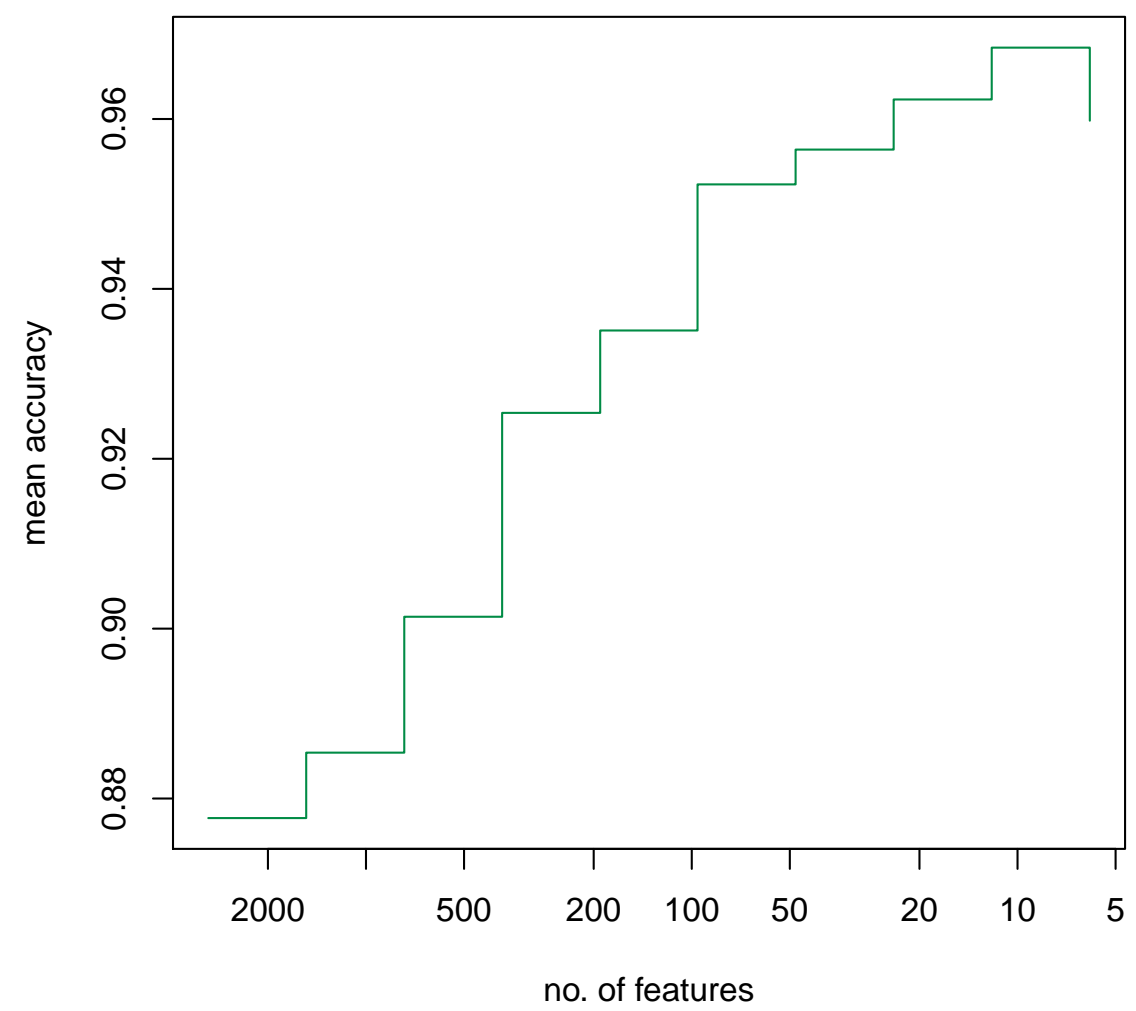

Figure 3.3: Mean accuracy change with the number of features for Golub leukemia data in the first stage

Figure 3.3 shows the mean accuracy increment with decreasing number of features using the Golub leukemia data in the first stage of feature selection.

Figure 3.4 shows the mean accuracy increment with decreasing number of features of the Golub leukemia data in the second stage of feature selection. From this figure, 


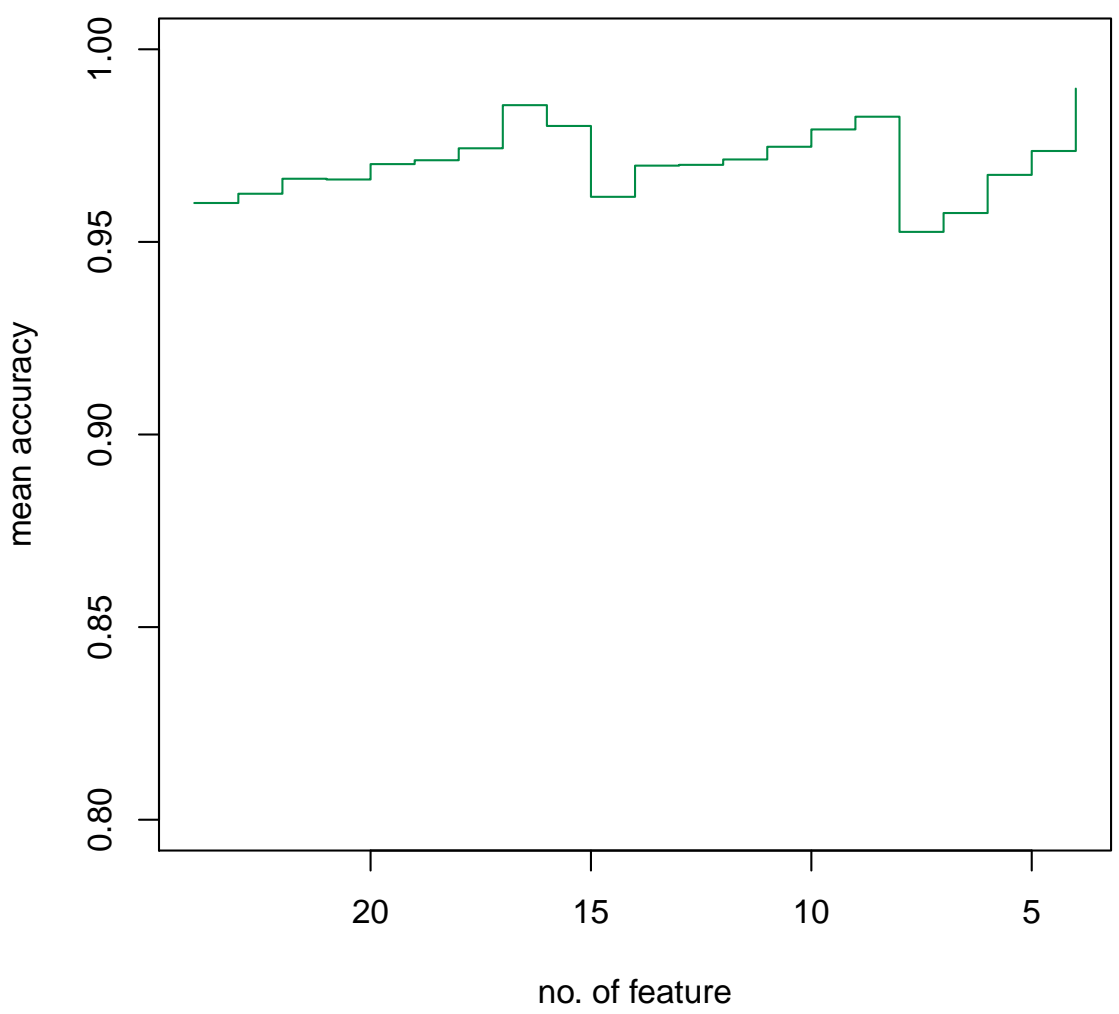

Figure 3.4: Mean accuracy change with the number of features for Golub leukemia data in the second stage (peak feature set is selected)

when 4 genes are left in the model, a maximum mean accuracy is reached. These 4 genes for leukemia classification are:

X95735_at, U27460_at, M27891_at, L09209_s_at

Now using these four genes and the ordinary KNN classifier $(k=3)$ to classify the 34 independent test samples, the confusion matrix is:

$\begin{array}{rrr}\text { classified as-> } & A L L & A M L \\ A L L & 18 & 2 \\ A M L & 1 & 13\end{array}$


Two ALL samples are classified as AML and one AML is classified as ALL. Total accuracy is as high as $91 \%$. This model is very simple compared with others that use far more genes.

\subsection{Comparisons with other methods}

Different classifiers for non-small cell lung cancer (NSCLC) using gene expression data were widely studied. The data set was published by Beer et al. in $2002^{111}$ and can be downloaded from NCI caArray database https://caarraydb.nci.nih.gov/ caarray/publicExperimentDetailAction.do?expId $=1015897558050283$. The array type is human GeneChip ${ }^{\circledR}$ Hu6800. Each chip contains 7,129 oligonucleotide probe sets. The complete data set includes 86 lung cancer tissue samples and 10 normal samples. 5-year survival classification through signature gene expression was studied. Normal subjects and those having operations less than 5 years ago and who are still alive were excluded. Thus, 43 samples are left. These 43 samples are further classified low and high risk groups, i.e., if the subject survived at least 5 years.

We applied our Random KNN approach on this data set. The class definition is the same. We first removed 58 control gene probe sets with prefix "AFFX". The two-stage Random KNN feature selection method is used. We get a set of four genes (see Table 3.1. Gene symbols are obtained from $\mathrm{R}$ annotation package hu6800.db). The RFNT1 is a recommended symbol. The original data file used alias "KIAA0084".

Table 3.1: Four genes selected by Random KNN for lung cancer survival prediction

\begin{tabular}{l|l|l}
\hline Probe ID & Gene Name & Gene Symbol \\
\hline \hline K02574_at & nucleoside phosphorylase & NP \\
D42043_at & raftlin, lipid raft linker 1 & RFTN1 \\
X86691_at & chromodomain helicase DNA binding protein 4 & CHD4 \\
L06132_at & voltage-dependent anion channel 1 & VDAC1 \\
\hline
\end{tabular}


Table 3.2 shows our improved result using the above 4 genes from our Random KNN method along with other results using 6 genes ${ }^{52}$ based on leave-one-out crossvalidation. One can see that our Random KNN method gives higher accuracy than others.

Table 3.2: Classification results of machine learning algorithms on lung cancer survival

\begin{tabular}{l|c}
\hline Method & Overall Accuracy \\
\hline \hline Proposed Random KNN & $88.4 \%$ \\
\hline Bayesian Networks & $76.7 \%$ \\
Decision Tree (ADTree) & $76.7 \%$ \\
Logit Boost & $76.7 \%$ \\
Logistic Regression & $72.1 \%$ \\
Neural Networks & $72.1 \%$ \\
K Nearest Neighbor (IBk) & $69.8 \%$ \\
Dempster-Shafer Networks & $83.7 \%$ \\
\hline
\end{tabular}

Gene information can be obtained from http://www.genecards.org. By this database, the NP gene "encodes an enzyme which reversibly catalyzes the phosphorolysis of purine nucleosides. The enzyme is trimeric, containing three identical subunits. Mutations which result in nucleoside phosphorylase deficiency result in defective T-cell (cell-mediated) immunity but can also affect B-cell immunity and antibody responses. Neurologic disorders may also be apparent in patients with immune defects. A known polymorphism at aa position 51 that does not affect enzyme activity has been described. A pseudogene has been identified on chromosome 2." Thus this gene relates to host immune function.

The RFTN1 gene "May play a pivotal role in the formation and/or maintenance of lipid rafts. May regulate B-cell antigen receptor-mediated signaling".

The CHD4 gene: "The product of this gene belongs to the SNF2/RAD54 helicase family. It represents the main component of the nucleosome remodeling and deacetylase complex and plays an important role in epigenetic transcriptional repression. 
Patients with dermatomyositis develop antibodies against this protein. Function: Probable transcription regulator".

The VDAC1 gene "Forms a channel through the mitochondrial outer membrane and also the plasma membrane. The channel at the outer mitochondrial membrane allows diffusion of small hydrophilic molecules; in the plasma membrane it is involved in cell volume regulation and apoptosis. It adopts an open conformation at low or zero membrane potential and a closed conformation at potentials above $30-40 \mathrm{mV}$. The open state has a weak anion selectivity whereas the closed state is cation-selective. May participate in the formation of the permeability transition pore complex (PTPC) responsible for the release of mitochondrial products that triggers apoptosis." Abnormal apoptosis will result in cell abnormal proliferation (cancer).

In summary, although we, as non-biologists, may not completely understand the pathway of these genes, at least they are cancer-related genes. Using these four genes, 38 are correctly classified, two high risk subjected are classified as low risk and three good prognoses classified into the opposite category. The accuracy is $88.4 \%$ and higher than all other methods in Table 3.2,

\subsection{Testing Random KNN on Other Data Sets}

\subsubsection{Test on Cancer Gene Expression Data Sets}

The Random KNN variable selection procedure was tested on 8 additional microarray data sets as listed in Table 2.3. Results are given in Table 3.3 .

In this table, classification accuracies by KNN using all genes are included as a reference. Random KNN feature selection was applied and 4 genes were selected for each data set. The classification accuracies using 4 genes are given (detailed classifi- 
Table 3.3: Classification accuracies (\%) on microarray data sets

\begin{tabular}{l|c|c|c|c}
\hline Data Set & KNN & Random Forest & RKNN & RKNN (4 genes) \\
\hline \hline Adenocarcinoma & 85.5 & 87.5 & 86 & 89.5 \\
Brain & 76 & 85 & 76 & 93 \\
Breast (2 classes) & 62 & 66 & 60 & 86 \\
Breast (3 classes) & 56 & 65 & 55 & 73 \\
Colon & 77 & 87 & 84 & 89 \\
Lymphoma & 98 & 99 & 98 & 98 \\
Prostate & 83 & 92 & 88 & 92 \\
SRBCT & 97 & 98 & 98 & 95 \\
\hline Average & 79 & 85 & 81 & 89 \\
\hline
\end{tabular}

cations are in the next section). From this table one can see that (1) the classification accuracies using 4 genes are high; (2) for data sets whose KNN classification accuracies are $<85 \%$ using all the genes, using 4 genes selected by Random KNN increases accuracies significantly. For the Lymphoma and SRBCT data sets, the accuracies are not improved by using Random KNN feature selection since the full models already get very high accuracies.

Random Forests was also used to select important genes for these data sets ${ }^{32}$. For 5 of the 8 data sets, Random KNN's performances are comparable to Random Forest, and on the other 3 data sets Random KNN performed significantly better.

\subsubsection{Detailed Testing Results}

The first stage of our backward elimination procedure with Random KNN was applied to the 8 data sets and 4 genes are selected for each data set. The confusion matrices obtained by Leave-One-Out Cross-validation (LOOCV), which provides good estimators of generalization error for stable classifiers such as $\mathrm{KNN}^{24}$, are given below (with accuracy rate in parentheses):

Adenocarcinoma data (89.5\%): 


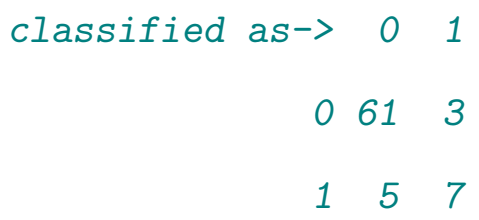

Brain data $(93 \%)$ :

$\begin{array}{rrrrrr}\text { classified as }-> & 0 & 1 & 2 & 3 & 4 \\ 0 & 10 & 0 & 0 & 0 & 0 \\ 1 & 0 & 10 & 0 & 0 & 0 \\ 2 & 0 & 0 & 10 & 0 & 0 \\ 3 & 0 & 0 & 0 & 4 & 0 \\ 4 & 1 & 0 & 1 & 1 & 5\end{array}$

Breast (2 classes) data (86\%):

$$
\begin{array}{rrr}
\text { classified as }-> & 0 & 1 \\
0 & 28 & 5 \\
1 & 5 & 39
\end{array}
$$

Breast (3 classes) data (73\%):

$$
\begin{array}{rrrrr}
\text { classified as }-> & 0 & 1 & 2 \\
0 & 14 & 1 & 3 \\
1 & 2 & 33 & 9 \\
2 & 2 & 9 & 22
\end{array}
$$

$\underline{\text { Colon data }}(89 \%)$ :

$\begin{array}{rrr}\text { classified as }-> & \text { Normal } & \text { Tumor } \\ \text { Normal } & 19 & 3 \\ \text { Tumor } & 4 & 36\end{array}$


Lymphoma data (98\%):

$\begin{array}{rrrrr}\text { classified as }> & 0 & 1 & 2 \\ 0 & 41 & 0 & 1 \\ 1 & 0 & 9 & 0 \\ 2 & 0 & 0 & 11\end{array}$

Prostate data $(92 \%)$ :

classified as $\rightarrow \quad 0 \quad 1$

0464

1448

$\underline{\text { Small round blue cell data }}(95 \%)$ :

$\begin{array}{rrrrrr}\text { classified as }-> & 0 & 1 & 2 & 3 \\ 0 & 23 & 0 & 0 & 0 \\ 1 & 0 & 17 & 1 & 2 \\ 2 & 0 & 0 & 12 & 0 \\ 3 & 0 & 0 & 0 & 8\end{array}$

\subsection{Stability of Random KNN}

Since randomness is introduced in the feature selection procedure, the stability of this procedure is of concern. By stability, we mean the repeatability of class assignment of a given sample by the different classifiers constructed by our Random KNN procedure without or with feature selection. To test the stability, we repeated the procedure 30 times over the 8 microarray data sets and got 30 classifiers for each data sets before and after feature selection. The accuracies of the 30 classifiers are calculated. Box 
plots are presented in Figures 3.5 and 3.6. The coefficient of variability (ratio of standard deviation to mean) of the classifiers are also computed and listed in Table 3.4. We can see that there are some variability associated with the random procedure. Remember that even for those deterministic procedures, randomness and therefore variability can not be avoided since data are random samples. In fact, this variability for our procedure is rather small. The coefficient of variability is not greater than $5 \%$ $(k=3, m=\lfloor\sqrt{p}\rfloor, r=500$ for Random KNN and $r=2000$ and 500 for stage I and stage II feature selection, respectively).

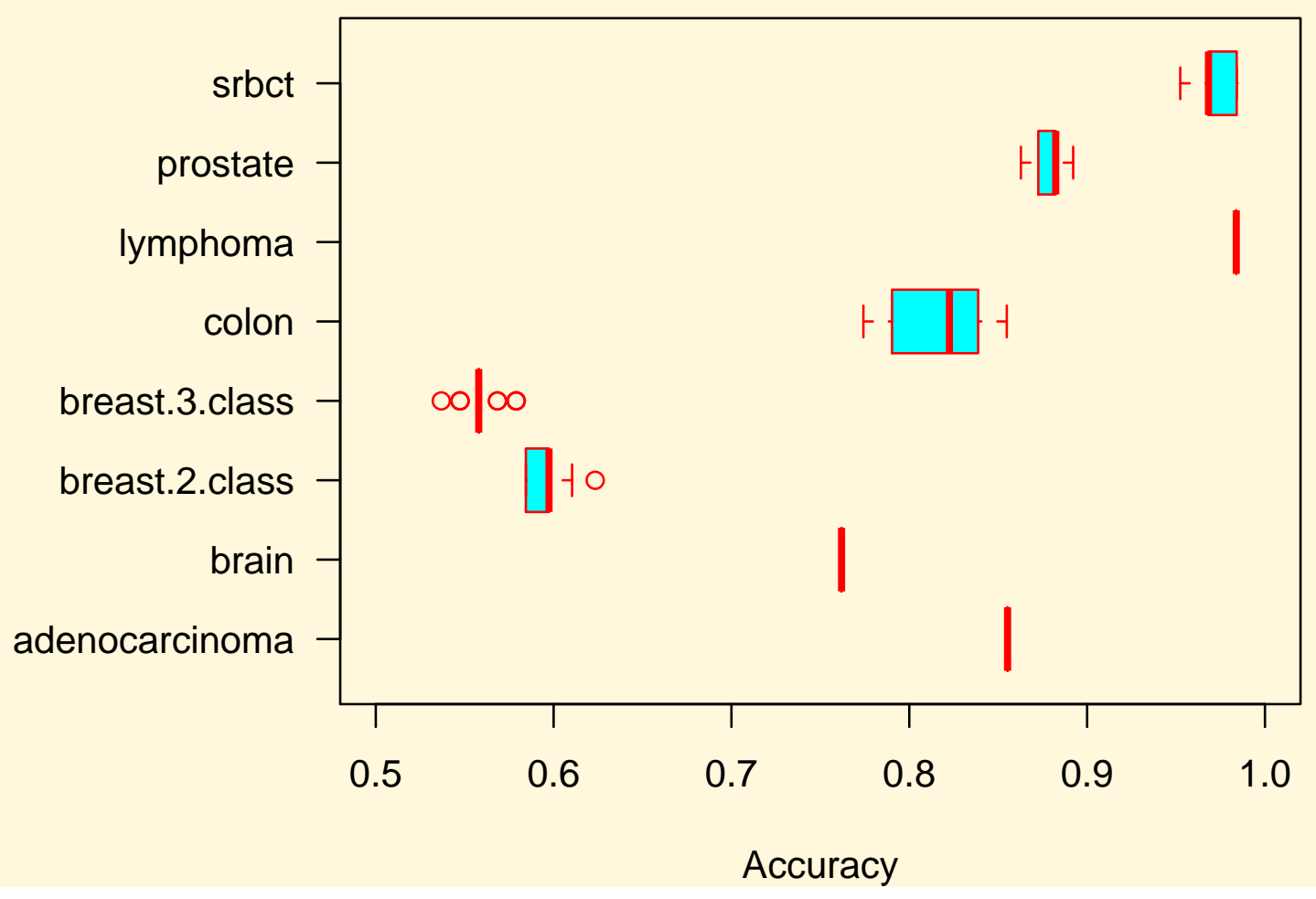

Figure 3.5: Box plots of Random KNN accuracy before feature selection 


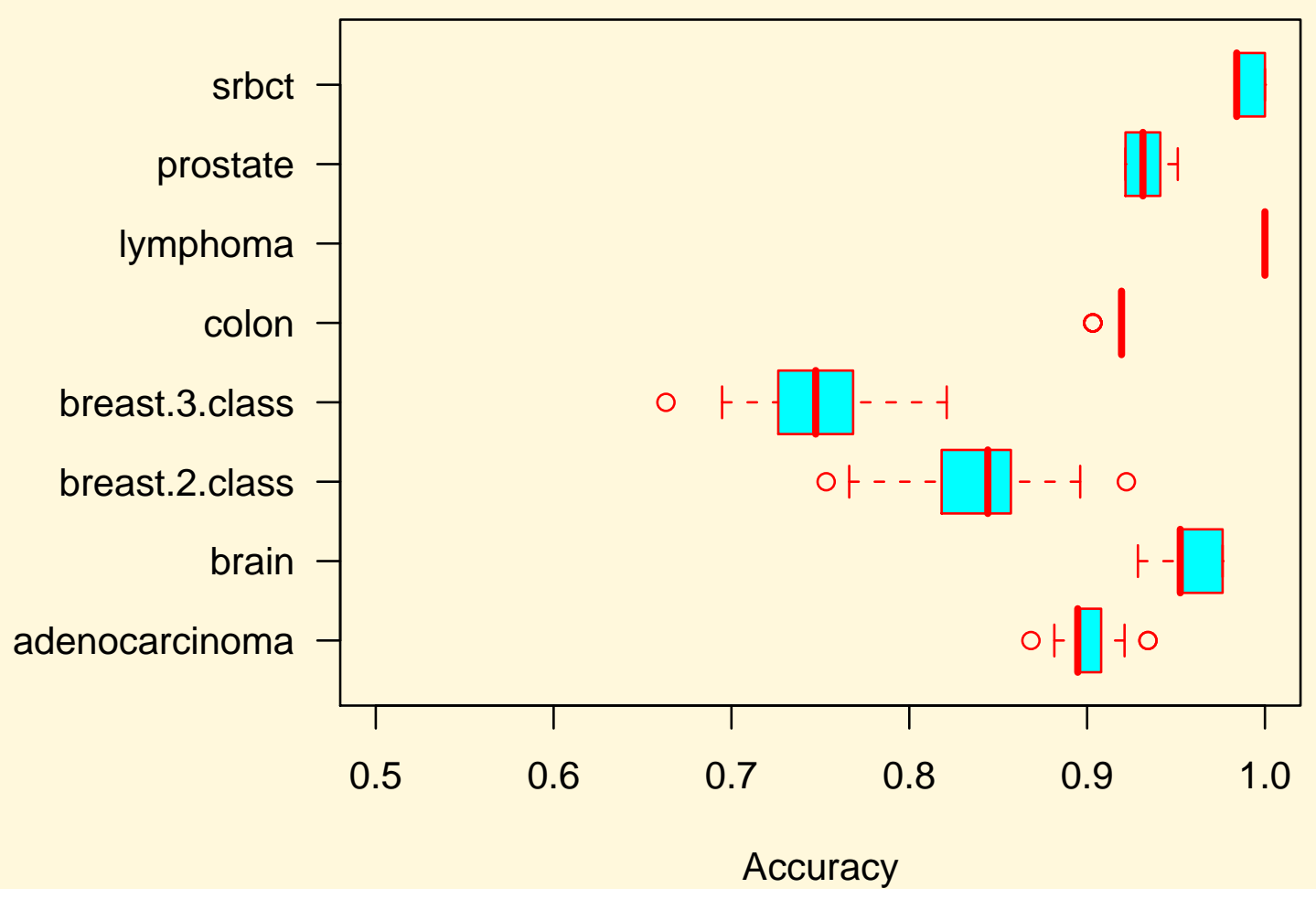

Figure 3.6: Box plots of Random KNN accuracy after feature selection

Table 3.4: Coefficient of variability of Random KNN classification accuracies of 30 runs

\begin{tabular}{l|c|c}
\hline Data Set & Before Feature Selection & After Feature Selection \\
\hline \hline adenocarcinoma & 0.00 & 0.02 \\
brain & 0.00 & 0.02 \\
breast (2 classes) & 0.02 & 0.05 \\
breast (3 classes) & 0.02 & 0.04 \\
colon & 0.03 & 0.01 \\
lymphoma & 0.00 & 0.00 \\
prostate & 0.01 & 0.01 \\
srbct & 0.01 & 0.01 \\
\hline
\end{tabular}

\subsection{Discussion}

We presented a two-stage backward elimination procedure for feature selection. This procedure is easy to implement. We also explored the possibility of combining Ran- 
dom KNN with other more complex searching algorithms to select features for high dimensional data, like the so-called floating search ${ }^{93}$ (95|103, which seems to have an appealing feature that is claimed to prevent the nesting effect. However, there are several impediments to applying these try-and-error algorithms comprised with "inclusion" and "exclusion" base steps. One is the large amount of computation. Suppose that at some point, $s$ of $p$ features are selected, to try to include an additional feature, the remaining $p-s$ features need to be evaluated with respect to the $q$ features. That is $p-s$ Random KNN models based on feature sets of size $s+1$ are needed to be built. Similarly, to try to exclude one from the $s$ features, $s$ models are needed to be built on $s-1$ features. This is quite computational-intensive and not suitable when $p$ is large. Another hurdle is that the implementation of this algorithm is very complex. Overall, the one-way search method as proposed is more practical for high dimensional data, e.g., gene expression, etc.

As was earlier observed by other investigators, even using simpler classifier, for example, the ordinary KNN classifier, the floating search "is not recommended for data sets with a large number of attributes (say more than 1000)" We used the sequential forward floating search (SFFS) with ordinary KNN on the Golub's Leukemia data (38 cases and 3,051 genes), 2,124 genes are selected as a "best subset". This result is disappointing because this subset is too large for this data set with only 38 cases.

In addition to feature selection, PCA dimension reduction may be applied. We choose first 3 principal components for each random subset of features and used two ways to conduct classification: (i) combine all the first 3 principal components, then perform KNN classification. That is PCA-Combine-KNN scheme; (ii) separately perform KNN classification using the 3 principal components of each random subset of features, then combine the results. That is PCA-KNN-Combine scheme. The classification results on 8 microarray data sets along with Random KNN (RKNN) method 
are given in Table 3.5. It can be seen that using principal components could get similar results as that using original features in Random KNN. This shows that most of the features are redundant, therefore, Random KNN feature selection improves the classification performances.

Table 3.5: Accuracies of PCA-KNN classification on microarray data sets

\begin{tabular}{l|c|c|c|c}
\hline Data Set & PCA-Combine-KNN & PCA-KNN-Combine & RKNN & RKNN (4 genes) \\
\hline \hline Adenocarcinoma & 87 & 84 & 86 & 89.5 \\
Brain & 83 & 71 & 76 & 93 \\
Breast (2 classes) & 61 & 60 & 60 & 86 \\
Breast (3 classes) & 59 & 54 & 55 & 73 \\
Colon & 76 & 79 & 84 & 89 \\
Lymphoma & 98 & 100 & 98 & 98 \\
Prostate & 81 & 81 & 88 & 92 \\
SRBCT & 97 & 95 & 98 & 95 \\
\hline Average & 80 & 78 & 81 & 89 \\
\hline
\end{tabular}




\section{Chapter 4}

\section{Random KNN Regression}

\subsection{Regression for High Dimensional Data}

Regression for high dimensional data is challenging and difficult, and impossible from the standpoint of the classical regression approach. The challenges come from several fronts: (i) The form of the parametric model is hard to specify. In most cases, in high dimensional space, the model will not be linear but rather a complex nonlinear model. The complexity of such nonlinear model is usually beyond our ability to discern the mathematical form, even in an approximate sense; (ii) The data set is usually too small compared with the dimensionality. Therefore even if the model can be specified in a linear form, the model parameter estimation is unattainable since the design matrix $X$ does not have full column rank and the quadratic form $X^{\prime} X$ is not of full rank either. Thus the singular quadratic form cannot be inverted to get the estimate of the parameters. Even using the generalized inverse, such as MoorePenrose pseudoinverse ${ }^{85191}$ through the singular value decomposition (SVD), $\left(X^{\prime} X\right)^{+}$ of $X^{\prime} X$, the solution to the normal equations is only one of many. Such a non-unique solution is not the estimator of the coefficients. To fix the lack of uniqueness, many 
extra constraints ${ }^{\sqrt{99}}$, which are not available in practice, must be applied; (iii) The error terms are not Gaussian. Any formal tests based on normality assumption are not applicable. Here it may be worth mentioning the problem of multicollinearity, i.e., the case where features are almost linearly dependent. With multicollinearity, the coefficient estimates of a linear model will not be stable, and small perturbation may result in quite different coefficient estimates. Although it will not be a problem as far as predictive accuracy is concerned ${ }^{59973}$, it will be problematic if we make any inference about the variables, for example, variable importance and ranking. Hence, nonparametric approaches for regression are unavoidable.

One type of nonparametric method is local fitting, either through smoothing splines, local linear, or the kernel weighting function. These methods fit a data point based on its neighborhood. For the same reasons, spline or local linear models are also limited to low dimensional data. For example, the loess algorithm in $\mathrm{R}$ can only use up to 4 predictors. On the other hand, the kernel method is connected to the $k$-nearest neighbor (KNN) method. The neighborhood for the kernel method is defined by the "band width" turning parameter. For high dimensional data, due to the sparseness of the data points, there may be no data point in many bands and thus the fitted surface will be bumpy, resulting in lack of fit. On the contrary, the KNN method is more adaptive to high dimensional data in that its bands are variable rather than fixed.

Let $\mathbb{N}_{i}$ be the $k$-nearest neighbor index set for point $x_{i}$, permitting self-match. Then the fitted value for $x_{i}$ is given by:

$$
\hat{y}_{i}=\sum_{j \in \mathbb{N}_{i}} w_{i j} y_{j},
$$

where $w$ is a weighting function such that the $k$ weights sum to 1 , usually choose 
$w=1 / k$. If $x_{i}$ itself is excluded when searching its nearest neighbors, defining such a $k$-nearest neighbor index set $\mathbb{N}_{i,-i}$ for point $x_{i}$ we get the predicted response:

$$
\hat{y}_{i,-i}=\sum_{j \in \mathbb{N}_{i,-i}} w_{i j} y_{j}
$$

Then we can define the predicted sum of square (PRESS) statistic as for the linear regression model:

$$
\begin{aligned}
\text { PRESS } & =\sum_{i=1}^{n}\left(y_{i}-\hat{y}_{i,-i}\right)^{2} \\
& =\sum_{i=1}^{n}\left(e_{i,-i}\right)^{2}
\end{aligned}
$$

where $e_{i,-i}$ are prediction errors or PRESS residuals. Since $y_{i}$ is not used for its estimate, the PRESS residual is independent of the training data. Furthermore, a normalized goodness-of-fit measure based on PRESS is a $R^{2}$ statistic:

$$
R_{P}^{2}=1-\frac{P R E S S}{\sum_{i=1}^{n}\left(y_{i}-\bar{y}\right)^{2}} .
$$

The measure $R_{P}^{2}$ can be a Leave-One-Out Cross-validation ( LOOCV) performance measure or an independent testing performance measure, depending on whether the estimate of $y_{i}$ is used to estimate for other data points. For example, if we have two data sets, $\mathbf{T}$ for training and $\mathbf{Q}$ for testing, and for each point $x_{i} \in \mathbf{Q}$, its neighbors are found only in $\mathbf{T}$, then $\mathbf{Q}$ is an independent testing data set and the corresponding $R_{P}^{2}$ is a performance measure independent of the training process. 


\subsection{Random KNN Regression}

As discussed above, KNN regression is a local, nonparametric method suitable for high dimensional data. In applications such as bioinformatics, the dimensionality could be very large, typically in the thousands. For example, with DNA microarray data, the number of genes is in the order of 10,000 , i.e., the dimension is quite high. So we may use a "local-of-local" approach, i.e., our Random KNN. With random KNN regression, we fit $r$ KNN regressions on $m$ randomly selected input features for each, and take the average prediction of these KNN regressions as our final prediction. Similarly we define $\mathbb{N}_{i, l}^{(m)}$ to be the $k$-nearest neighbor index set for point $x_{i}$ searched by using $m$ features for the $l$ th KNN regression, then

$$
\hat{y}_{i}=\frac{1}{r} \sum_{l=1}^{r} \sum_{j \in \mathbb{N}_{i, l}^{(m)}} w_{i j} y_{j} .
$$

The properties of the Random KNN discussed in chapter 2 also apply to the regression case.

\subsection{Application of Random KNN Regression to Drug Sensitivity}

Staunton at el. ${ }^{107}$ investigated chemosensitivity of a library of drug candidates using oligonucleotide microarrays (Affymetrix ${ }^{\circledR}$ ) of 60 cell lines in the NCI60 panel (see review paper ${ }^{101}$ and NCI web ${ }^{87}$ ). The gene expression and drug response data can be found from the authors' depository at the Broad Institute web site ${ }^{64}$. There are 6,817 genes in each array. This data set is also preprocessed and ported into $\mathrm{R}$ by Adeín Culhane and can be downloaded from their $\mathrm{R}$ package made4 web site ${ }^{30}$. This $\mathrm{R}$ 
version data set contains 1,517 genes for 60 samples. The drug responses are negative $\log$ of $\mathrm{GI}_{50}$, which is the drug concentration of a compound required for $50 \%$ growth inhibition. The drug Azaguanine with NCI's ID number NSC749 was illustrated by the authors. We take this drug response as our continuous dependent variable $y$. We will use the first 30 samples as training set and the remaining 30 samples as testing set. We fit $r=500 \mathrm{KNN}$ regression models using the default setting for $m$, which is the square-root of $p=1,517$. The following is the output:

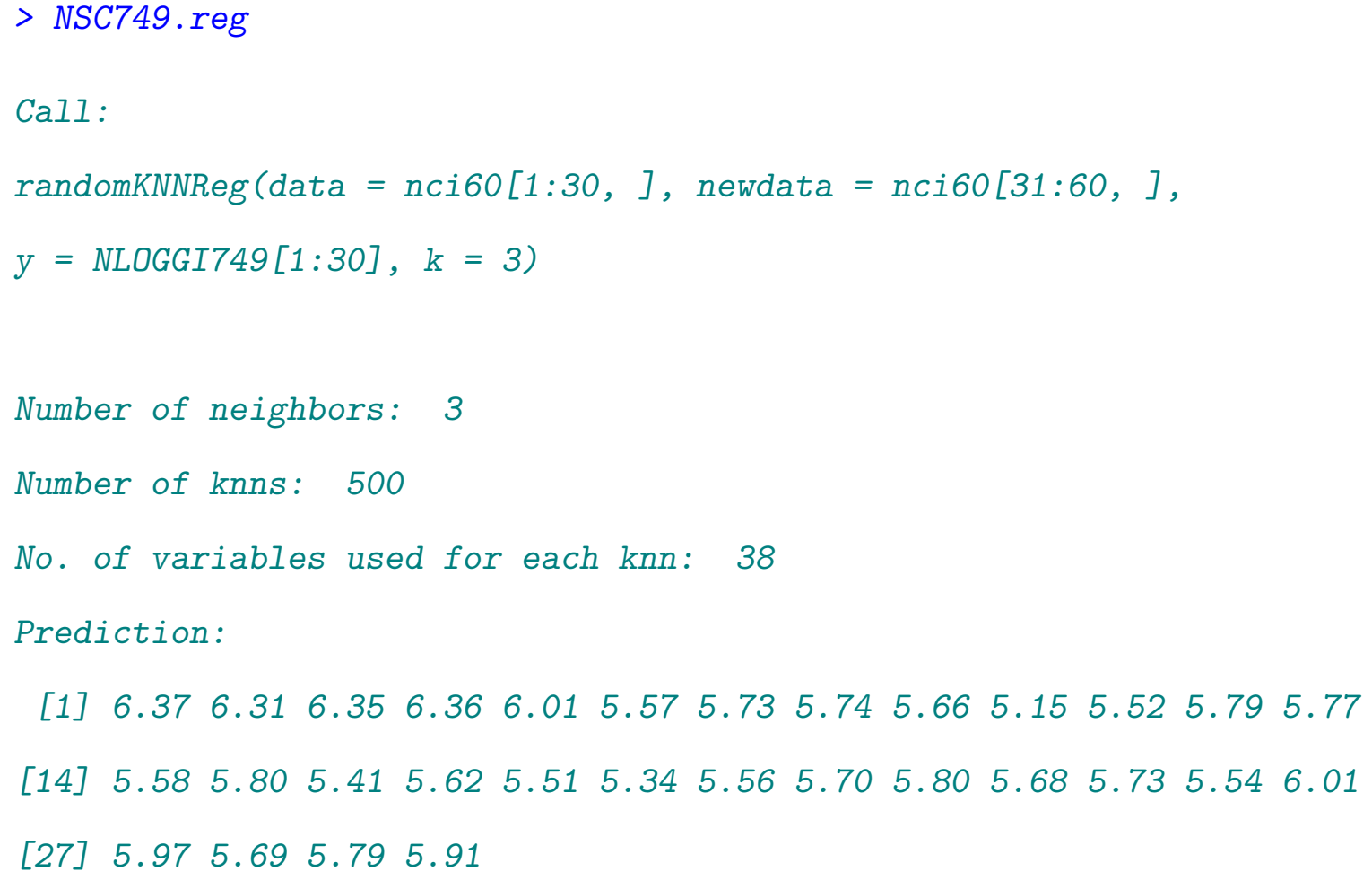

The PRESS statistic can be easily computed:

NSC749_PRESS

[1] 15.9

and $R_{P}^{2}=0.241$ 


\subsection{Feature Selection for Random KNN Regres- sion}

\subsubsection{Feature Ranking by Random KNN Regression}

As for least-square regression, there are many different models with inclusion of a different subset of regressor variables. To single out a good model from many, some criteria must be defined for KNN regression. We will adopt a similar approach as we did for the classification problem. We define a measure of "support" to sort all the features. To compute this measure, we fit a collection of random KNN regression models using randomly picked $m$ variables. For each model, $R_{P}^{2}$ is calculated by a (fixed or dynamic) splitting of the data into training and testing subsets. The role of $R_{P}^{2}$ is analogous to the accuracy measure for KNN classification. Thus the support can also be computed by an algorithm similar to Algorithm 3.1. As an example, we compute the support for the NCI60 drug response data.

First, the dynamic partition is used:

> NSC749_Support

Call:

supportKNNReg $($ data $=$ nci60, $\mathrm{y}=$ NLOGGI749, $\mathrm{k}=3$, fixed.partition $=$ FALSE)

Number of knns: 500

No. of variables used for each knn: 38

Accuracy: 0.212

Then, we use the fixed partition method: 
> NSC749_Support2

Call:

supportKNNReg $($ data $=$ nci60, $\mathrm{y}=$ NLOGGI749, $\mathrm{k}=3$, fixed.partition $=$ TRUE)

Number of knns: 500

No. of variables used for each knn: 38

Accuracy: 0.638

Figure 4.1 shows 30 genes with highest supports in the NCI60 drug response data using fixed and dynamic partitions.

\subsubsection{Feature Selection for Random KNN Regression}

Once the feature ranking criterion is computed, it can be used to remove the unimportant features from the data. The recursive elimination algorithm of section 3.2 (Algorithm 3.2) can be applied here, too. For the NCI60 drug data set, the first stage of the algorithm generated Figure 4.2. The minimum number of variables left is set to 50 .

From this figure, one can see that the mean accuracy (mean of $R_{P}^{2}$ ) increases as features are taken out. The feature set size is 83 for the prior-peak step. Now we continue from there to eliminate features one by one using the second stage algorithm.

Figure 4.3 is the plot from this algorithm. The figure shows that when 9 features are included, the accuracy is high. We use these 9 features as our final selection. The nine features are:

M69066_at, HG3523-HT4899_S_at, J03040_at, M13929_S_at, M31994_at, M63379_at, U36764_at, D14520_at, M38591_at 

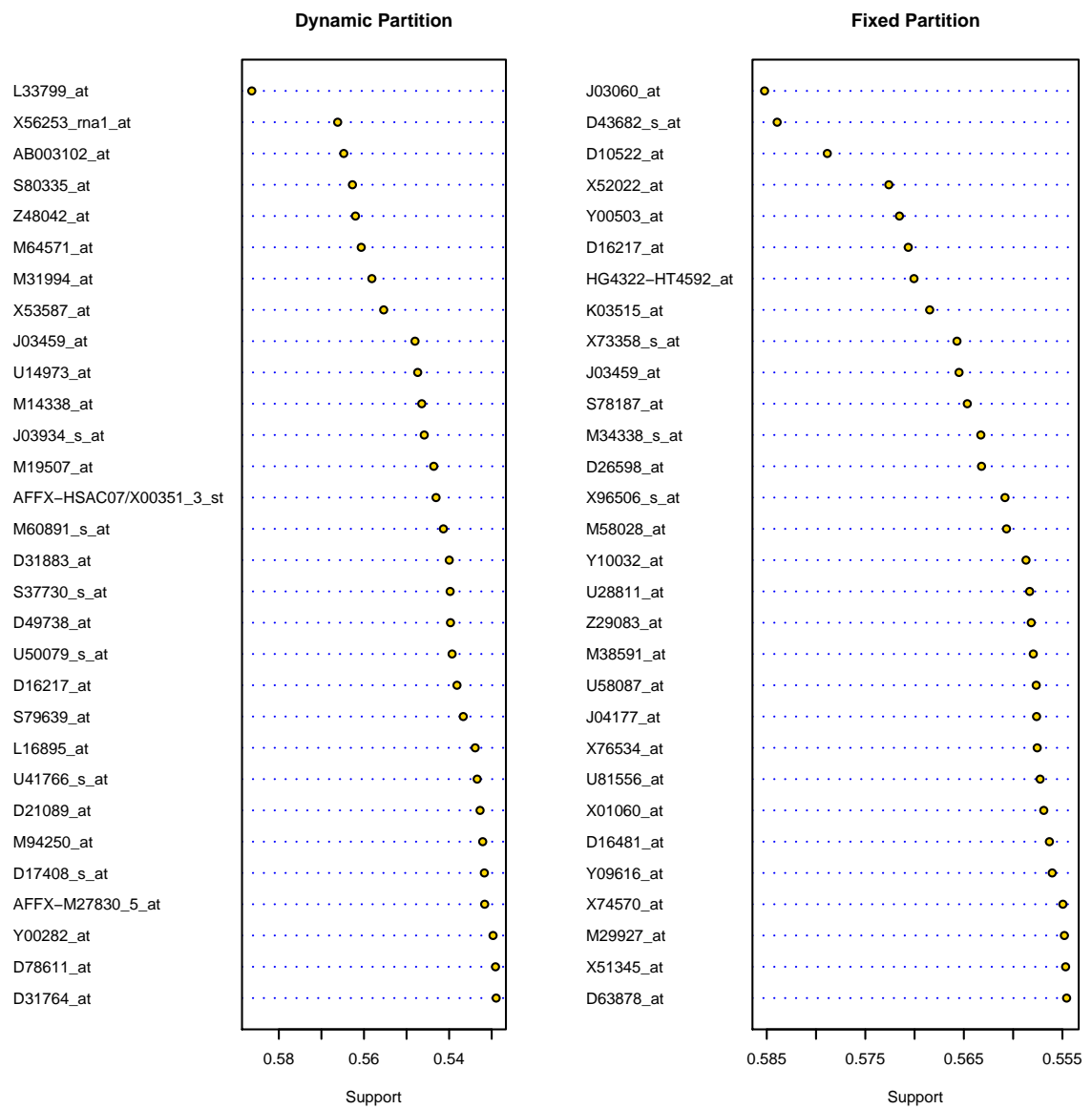

Figure 4.1: Supports of the top 30 genes of the NCI60 drug response data

Now use these 9 genes to build a (non-random) KNN regression and compute cross-validated PRESS and $R_{P}^{2}$ :

$>$ NSC749. CV2

PRESS $=12.3$

R2-Predict $=0.693$

To validate the effectiveness of the feature selection method, we may compare the result with the full mode:

> NSC749_full.cv

PRESS $=38.3$

R2-Predict $=0.0393$ 


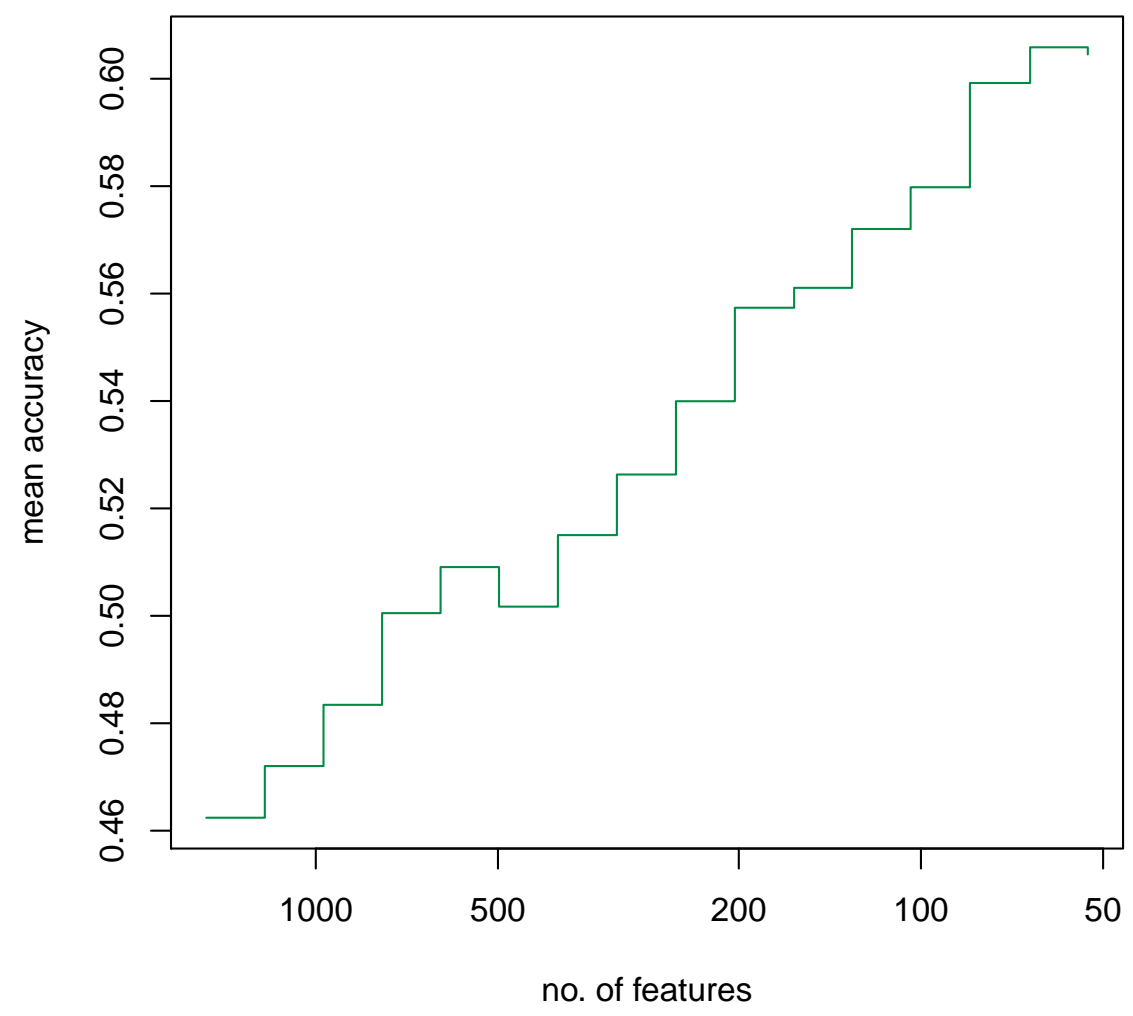

Figure 4.2: Mean accuracy change with the number of features for NCI60 drug data

It is indicated that the PRESS dropped sharply and the $R_{P}^{2}$ had a steep rise for the nine-gene model.

In addition, we can have graphical diagnosis of the KNN regression based on these genes. Figure 4.4 shows fitted values versus observed values and residuals versus fitted values for both the reduced model (9 variables) and the full mode (1,517 variables).

The upper panel for the fitted-observed plots clearly demonstrates that the reduced model has much better agreement between the fitted and the observed values than that for the full model. The (PRESS) residual plots on the lower panel also show that the reduced model gives a better fit. 


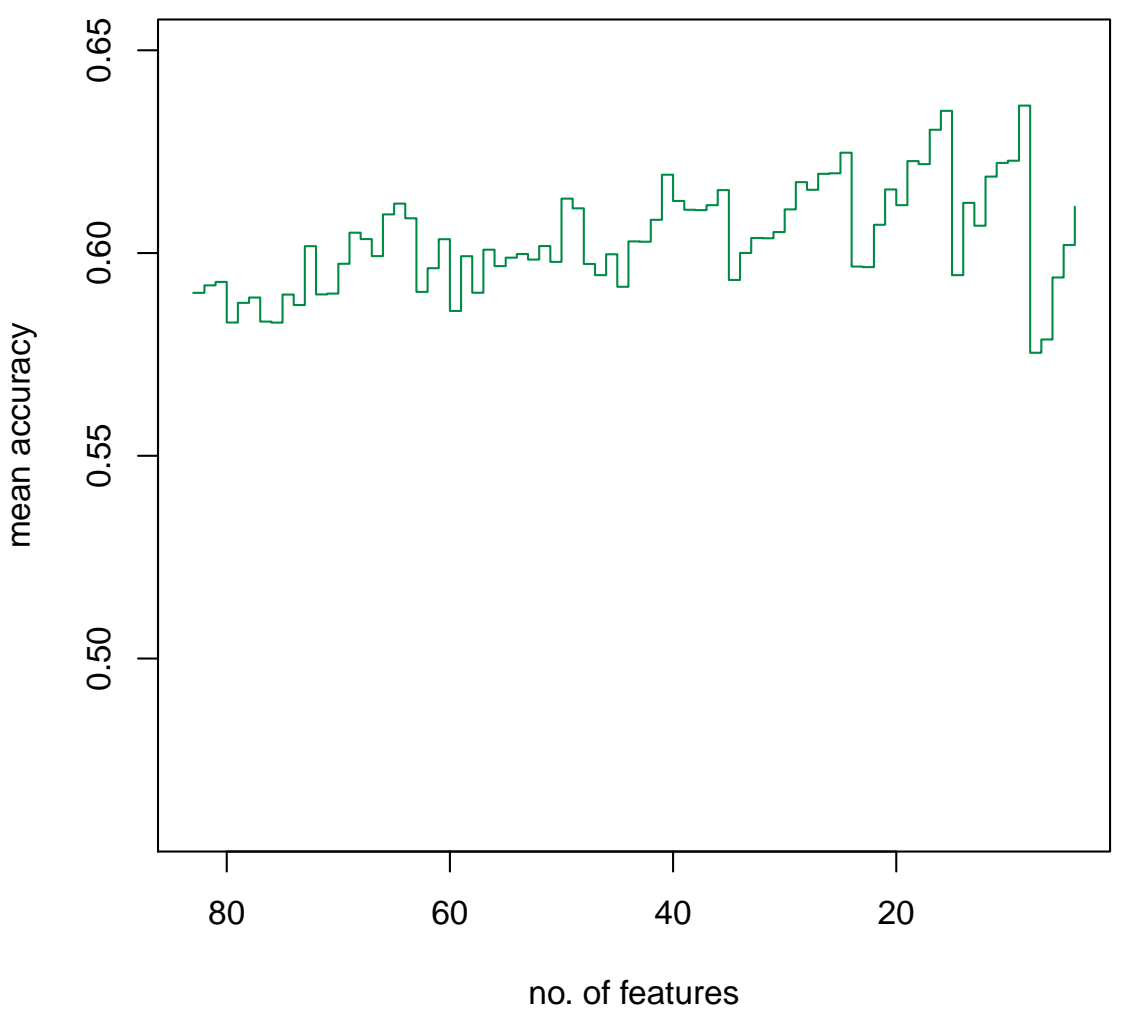

Figure 4.3: Mean accuracy change with the number of features for NCI60 drug data

From the above analysis, the Random KNN feature selection approach for KNN regression has the capability to choose important variables for such a nonparametric regression model. 


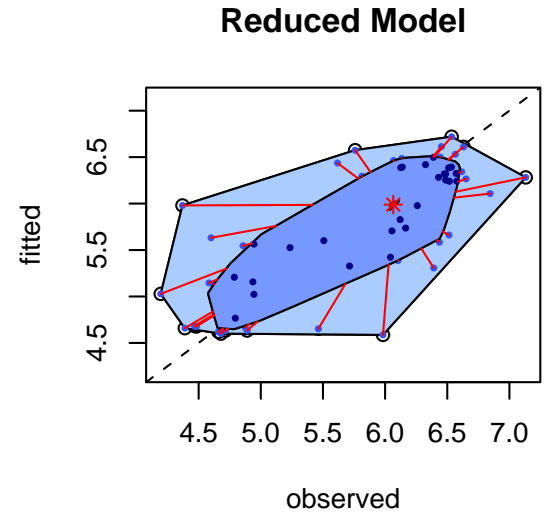

Reduced Model

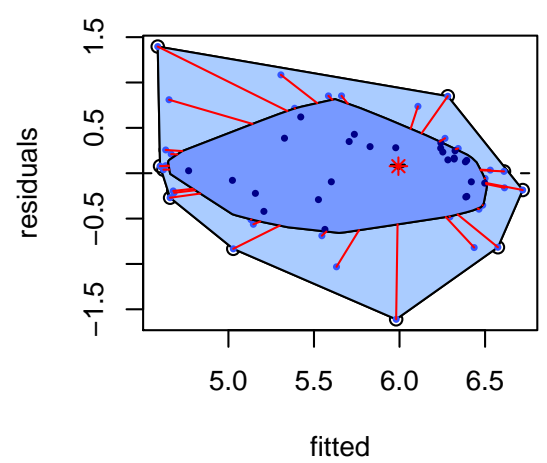

Full Model

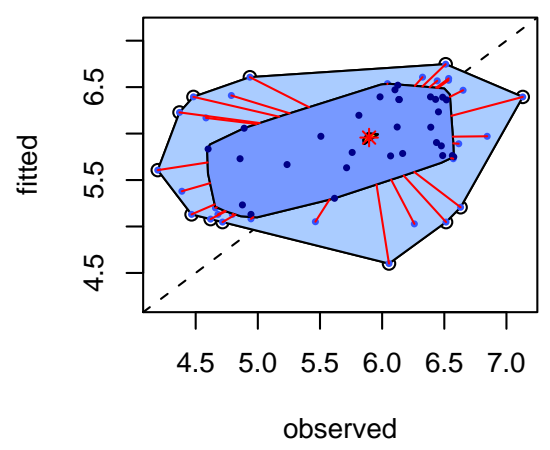

Full Model

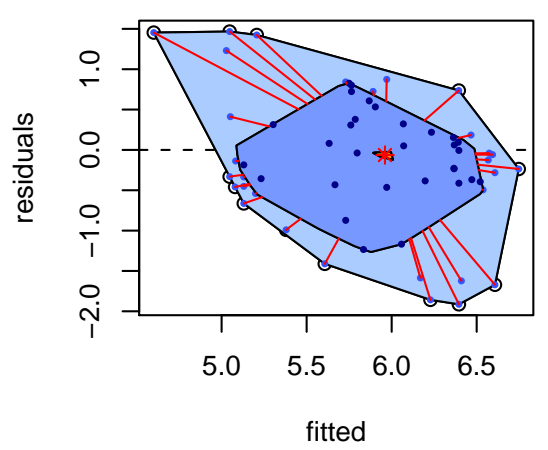

Figure 4.4: Diagnostic plots for KNN regressions (9 genes for reduced model and 1, 517 genes for full model)

\subsection{Comparison with the LASSO}

LASSO, standing for "least absolute shrinkage and selection operator", is a model selection method proposed by Tibshirani 41111 . It's mainly used for linear models.

The NCI60 Drug Response Data: We applied LASSO to this data set and got 13 none-zero coefficient variables. These 13 genes include:

M63379_at, X03689_s_at, M37984_rna1_at, M31994_at, HG3342.HT3519_s_at, M38591_at, M69066_at, D31890_at, HG1862.HT1897_at, M34338_s_at, HG2917.HT3061_f_at, D14520_at, HG3523.HT4899_s_at

Based on these 13 variables, a linear model is fitted. The PRESS and $R_{P}^{2}$ are: 
$>\operatorname{PRESS}($ nci60.Im13)

[1] 13.8

$>\operatorname{rsqp}($ nci60.1m13)

[1] 0.655

These two performance measures are close to what we obtained by the 9 -variable KNN regression model. Figure 4.5 show the diagnostic plots.

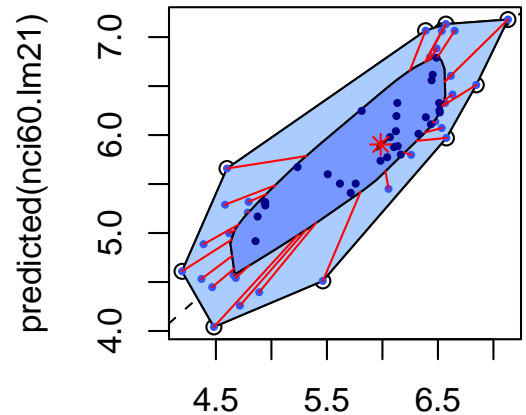

NLOGGI749

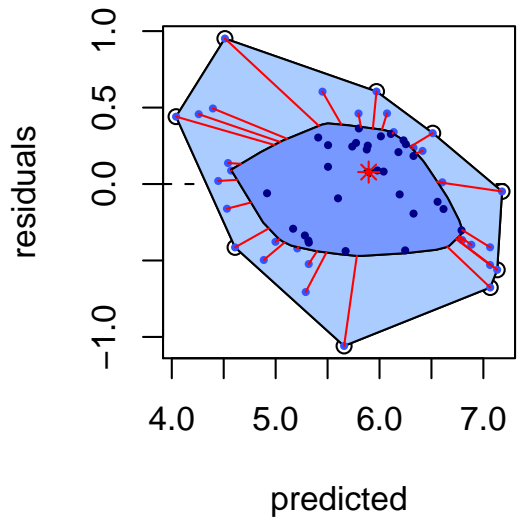

Figure 4.5: Diagnostic plots for the 13-variable linear model selected by LASSO

PAC Chemometrics Data: The PAC data set in the R package chemometrics contains GC-retention indices $(y)$ of 209 polycyclic aromatic compounds with 467 molecular descriptors (features). We first standardized the descriptors to zero mean and unit variance. Then our Random KNN regression feature selection and LASSO feature selection were applied. Random KNN selected 5 descriptors and the performance is:

PRESS $=58433.56$

R2-Predict $=0.957$ 
LASSO selected a set of 5 different descriptors and the PRESS and $R_{P}^{2}$ of its linear model are similar to above Random KNN method:

$>$ PRESS (PAC.1asSO.1m)

[1] 59839.72

$>\operatorname{rsqp}($ PAC.Iasso.Im)

[1] 0.956

The above results are tabulated in Table 4.1.

Table 4.1: Comparison of Random KNN regression with LASSO

\begin{tabular}{l|c|c|c|c}
\hline \multirow{2}{*}{$\begin{array}{l}\text { Data } \\
\text { Set }\end{array}$} & \multicolumn{2}{|c|}{ PRESS } & \multicolumn{2}{c}{$R_{P}^{2}$} \\
\cline { 2 - 5 } & Random KNN & LASSO & Random KNN & LASSO \\
\hline \hline NIC60 & 12.3 & 13.8 & 0.693 & 0.655 \\
PAC & 58434 & 59840 & 0.957 & 0.956 \\
\hline
\end{tabular}

In summary, Random KNN feature selection method for regression has comparable performance with the LASSO method based on these testing. In addition, Random KNN regression can also be used for missing value imputation by considering the variable with missing values as the dependent variable in regression and predicting the missing values from other variables. 


\section{Chapter 5}

\section{Conclusions and Future Work}

\subsection{Major Contributions}

In this dissertation, a new classification method, Random KNN, is developed for high dimensional data. Using Random KNN, a variable selection method for high dimensional data is proposed. Furthermore, a Random KNN regression method and the related model selection for high dimensional data are also developed.

Properties pertaining to Random KNN and the theoretical analysis of the Random KNN convergence for uncorrelated features are studied. In terms of complexity, Random KNN runs faster than ordinary KNN for high dimensional data. Using features selected by the proposed Random KNN method, higher classification accuracies than other methods are obtained. In summary, Random KNN approaches provide us both efficient and effective solutions to modeling high-dimensional data. 


\section{$5.2 \quad$ Limitations}

The convergence of Random KNN for correlated features is complex and difficult, therefore our theoretical analysis of Random KNN is limited for simplified situations and may not be realistic for practical applications.

\subsection{Future Research Directions}

Following the current work, in the future we will focus on more tests and experiments with the Random KNN approach, such as variable dropping schedule, step size, adaptivity, etc., and more comparisons for Random KNN classification and regression with other methods. In addition, after the first stage of feature selection, the number of features will be much smaller. Thus other exhaustive searching approach, e.g., branch and bound method, may be applicable. For some applications with prior knowledge, users may want to keep specific predictors - forced-in variables. The Random KNN feature selection algorithm may be extended to include this functionality. Another direction would be to investigate the potential of the Random KNN approach in studying gene to gene or protein-protein interaction networks. A putative gene interaction network may be built through Random KNN frame work by considering the predictive power of paired features or the random subsets of features used in Random KNN.

Improvements in performance may result if we consider the parameters of the model (namely, $m, k, r$ ) as random variables, rather than as fixed parameters in the Random KNN model. We have assumed that these parameters, once decided, assume the same value for the entire run of the Random KNN procedure. Another interesting future investigation would be to study the performance of the Random 
KNN approach when these parameters are allowed to vary during the same run of the system.

\subsection{Conclusions}

The proposed Random KNN is a novel approach for classification and regression for high dimensional data. It partially solves the curse of dimensionality problem. On one hand, it alleviates the exponential growth of the running time of the ordinary KNN method. On the other hand, it also attacks the need of exponential growth of samples in a high dimensional neighborhood by working in relatively lower dimensional space, and thus the variance will be reduced. The proposed variable selection method based on Random KNN can select important features and thus can be used to construct simple classification or regression models with good generalization performance. These approaches may be applied to bioinformatics study, especially microarray data analyses, to find differentially expressed genes highly related to a tumor or other phenotype. The Random KNN approach is a significant contribution to statistics, machine learning, pattern recognition and bioinformatics.

Through various testing, the Random KNN was shown to be an easy to use method. Although there are multiple moving parts such as $r, m$ and $k$, they can be readily set,e.g., taking $m$ be the square-root of $p$, searching for $k$ by validation, and choosing sufficient large $r(>200)$. 


\section{Bibliography}

1. Edgar Acuña. dprep package manual: Data preprocessing and visualization functions for classification, 2008. URL http://math.uprm. edu/^edgar/dprep. html. 58

2. Edgar Acuña and Caroline Rodriguez. The treatment of missing values and its effect in the classifier accuracy. In D. Banks, L. House, F.R. McMorris, P. Arabie, and W. Gaul, editors, Classification, Clustering and Data Mining Applications, pages 639-648. Springer-Verlag, Berlin-Heidelberg, 2004. 6

3. Edgar Acuña, F. Coaquira, and M. Gonzalez. A comparison of feature selection procedures for classifiers based on kernel density estimation. In Proceedings of the International Conference on Computer, Communication and Control technologies, CCCT'03., volume I, pages 468-472, 2003. 4

4. Hirotugu Akaike. A new look at the statistical model identification. IEEE Transactions on Automatic Control, 19(6):716-723, 1974. 4

5. Ahmed Al-Ani, Mohamed Deriche, and Jalel Chebil. A new mutual information based measure for feature selection. Intelligent Data Analysis, 7(1):43-57, 2003. 4 
6. Ilan Alder and Sheldon M. Ross. The coupon subsets collection problem. Journal of Applied Probability, 38(3):736-746, 2001. 14, 16

7. Sunil Arya, David M. Mount, Nathan S. Netanyahu, Ruth Silverman, and Angela Y. Wu. An optimal algorithm for approximate nearest neighbor searching in fixed dimensions. Journal of the ACM, 45(6):891-923, 1998. 25

8. Vassilis Athitsos, Jonathan Alon, Stan Sclaroff, and George Kollios. BoostMap: An embedding method for efficient nearest neighbor retrieval. IEEE Transactions on Pattern Analysis and Machine Intelligence, 30(1):89-104, January 2008. 25

9. D.A. Battaglia and L. Stella. Optimization through quantum annealing: theory and some applications. Contemporary Physics, 47(4):195-208, 2006. 5

10. N. Beckmann, H. Kriegel, R. Schneider, and B.Seeger. The ${ }^{*}$-tree: An efficient and robust access method for points and rectangles. In Proceeding of ACM SIGMOD, pages 322-331, Atlantic City, NJ, May 1990. 25

11. David G. Beer, Sharon L.R. Kardia, Chiang-Ching Huang, Thomas J. Giordano, Albert M. Levin, David E. Misek, Lin Lin, Guoan Chen, Tarek G. Gharib1and Dafydd G. Thomas, Michelle L. Lizyness, Rork Kuick, Satoru Hayasaka, Jeremy M.G. Taylor, Mark D. Iannettoni, Mark B. Orringer, and Samir Hanash. Gene-expression profiles predict survival of patients with lung adenocarcinoma. Nature Medicine, 8(8):816-824, August 2002. 50

12. Richard Ernest Bellman. Adaptive Control Processes: A Guided Tour. Princeton University Press, Princeton, NJ, 1961. 2 
13. J.L. Bentley. Multidimensional binary search trees used for associative searching. Communicatons of the ACM, 18(9):509-517, 1975. 25

14. J.L. Bentley. Multidimensional binary search trees in database application. IEEE Transactions on Software Engineering, (4):333-334, 1979.

15. J.L. Bentley. Multidimensional divide-and-conquer. Communicatons of the ACM, (4):214-229, 1980.

16. J.L. Bentley and B.W. Weide. Optimal expected-time algorithms for closest point problems. ACM Transactions on Mathematics Software, (4):563-580, 1980. 25

17. A. Beygelzimer, S. Kakade, and J. Langford. Cover trees for nearest neighbor, 2005. URL http://www.hunch.net/ jl/projects/covertree/paper/paper. ps. 25

18. A. Beygelzimer, S. Kakade, and J. Langford. Cover trees for nearest neighbor. In ICML '06: Proceedings of the 23rd international conference on Machine learning, pages 97-104, New York, NY, 2006. ACM Press. 25

19. J. Biesiada and W. Duch. Feature selection for high-dimensional data: A kolmogorov-smirnov correlation-based filter. In Computer Recognition Systems. Springer, 2005. 4

20. A. Blum and P. Langley. Selection of relevant features and examples in machine learning. Artificial Intelligence, 97(1-2):245-271, 1997. 3, 4

21. Leo Breiman. Random forests. Machine Learning, 45(1):5-32, 2001. 3, 9 
22. Leo Breiman. Manual-Setting Up, Using, And Understanding Random Forests, v4.0 edition, 2003. URL ftp://ftp.stat.berkeley.edu/pub/users/breiman/ Using_random_forests_v4.0.pdf. 11

23. Leo Breiman. Rf/tools: A class of two eyed algorithm, 2003. URL http: //oz.berkeley.edu/users/breiman/siamtalk2003.pdf, 3, 9

24. Leo Breiman. Heuristics of instability and stabilization in model selection. The Annals of Statistics, 24(6):2350-2383, 1996. 53

25. Leo Breiman. Bagging predictors. Machine Learning, 24:123Ü140, 1996. 12

26. Leo Breiman, Jerome Friedman, Charles J. Stone, and R.A. Olshen. Classification and Regression Trees. Chapman \& Hall/CRC, 1984. 3, 10

27. O. Chapelle and V. Vapnik. Model selection for support vector machines. In Advance in Neural Information Processing Systems, volume 12, Cambridge, MA, 2000. MIT Press. 5

28. T.M. Cover and P.E. Hart. Nearest nieghbor pattern classification. IEEE Transaction on Information Theory, IT-13(1):21-27, 1967. 5, 19

29. Nicholas L. Crookston and Andrew O. Finley. yaImpute: An R package for kNN imputation. Journal of Statistical Software, 23(10):1-16, 10 2007. 6

30. Aedín C. Culhane. made4 full datasets. URL http://bioinf .ucd.ie/people/ aedin/R/full_datasets/. 63

31. Belur V. Dasarathy, editor. Nearest Neighbor (NN) Norms: NN Pattern Classification Techniques. IEEE Computer Society Press, Los Alamitos, CA, 1991. 5 
32. Ramón Díaz-Uriarte and Sara Alvarez de Andrés. Gene selection and classification of microarray data using random forest. BMC Bioinformatics, 7(3), 2006. 11, 53

33. Ramón Díaz-Uriarte and Sara Alvarez de Andrés. Supplementary material for "gene selection and classification of microarray data using random forest", 2006. URL http://ligarto.org/rdiaz/Papers/rfVS/rfVarSelSuplMat.pdf. 35

34. Ramón Díaz-Uriarte and Sara Alvarez de Andrés. Data files, 2006. URL http: //ligarto.org/rdiaz/Papers/rfVS/data.sets.zip. 35

35. Marcel Dettling and Peter Bühlmann. Boosting for tumor classification with gene expression data. Bioinformatics, 19(9):1061-1069, 2003. 35

36. Luc Devroye. Necessary and sufficient conditions for the pointwise convergence of nearest neighbor regression function estimates. Probability Theory and Related Fields, 61(4):467-481, 1982. 29

37. Luc Devroye, Laszlo Gyorfi, Adam Krzyzak, and Gabor Lugosi. On the strong universal consistency of nearest neighbor regression function estimates. The Annals of Statistics, 22(3):1371-1385, 1994. 30

38. Thomas G. Dietterich. Machine-learning research: Four current directions. The AI Magazine, 18(4):97-136, 1998. 12

39. Richard O. Duda, Peter E. Hart, and David G. Stork. Pattern Classification. John Wiley \& Sons, New York, 2000. 3

40. Sandrine Dudoit, Jane Fridlyand, and Terence P. Speed. Comparison of discrimination methods for the classification of tumors using gene expression data. Journal of the American Statistical Association, 97(457):77-87, 2002. 38 
41. Bradley Efron, Trevor Hastie, Iain Johnstone, and Robert Tibishirani. Least angle regression. The Annals of Statistics, 32(2):407-451, April 2004. 70

42. E. Fix and J. Hodges. Discriminatory analysis-nonparametric discrimination: Consistency properties. Technical Report 21-49-004, 4, US Air Force, School of Avaiation Medicine, 1951. 5, 29

43. H. Fröhlich and Zell A. Feature subset selection for support vector machines by incremental regularized risk minimization. In 2004 IEEE International Joint Conference on Neural Networks. IEEE, 2004. 5

44. J.H. Friedman, J.L. Bentley, and R.A. Finkel. An algorithm for finding best matches in logarithmic expected time. ACM Transactions on Mathematics Software, 3(3):209-226, 1977. 25

45. I. Gargantini. An effective way to represent quadtrees. Communicatons of the ACM, 25(12):905-910, December 1982. 25

46. A. Gionis, P. Indyk, and R. Motwani. Similarity search in high dimensions via hashing. In Proceedings of the 25th Very Large Database (VLDB) Conference, 1999. URL http://people.csail.mit.edu/indyk/vldb99.ps. 25

47. Alan M. Gittelsohn. An occupancy problem. The American Statistician, 23(2): $11-12,1969.14$

48. David E. Goldberg. Genetic Algorithms in Search, Optimization, and Machine Learning. Addison-Wesley Professional, 1989. 5

49. T.R. Golub, D.K. Slonim, P. Tamayo, C. Huard, M. Gaasenbeek, J.P. Mesirov, H. Coller, M.L. Loh, J.R. Downing, M.A. Caligiuri, C.D. Bloomfield, and E.S. 
Lander. Leukemia data. URL http://www-genome.wi.mit.edu/mpr/data_ set_ALL_AML.html. 36

50. T.R. Golub, D.K. Slonim, P. Tamayo, C. Huard, M. Gaasenbeek, J.P. Mesirov, H. Coller, M.L. Loh, J.R. Downing, M.A. Caligiuri, C.D. Bloomfield, and E.S. Lander. Molecular classification of cancer: class discovery and class prediction by gene expression monitoring. Science, 286:531-537, 1999. URL http://www-genome.wi.mit.edu/cgi-bin/cancer/datasets.cgi. 36

51. Y. Grandvalet and S. Canu. Adaptive scaling for feature selection in svms. In Advance in Neural Information Processing Systems, volume 15, Cambridge, MA, 2003. MIT Press. 5

52. Lan Guo group. Private communicaton: A novel computational framework for individualized clinical decision-making, 2007. 51

53. A. Guttman. R-trees: A dynamic index structure for spatial searching. In Proceeding of ACM SIGMOD, pages 47-57, June 1984. 25

54. I. Guyon and A. Elisseeff. An introduction to variable and feature selection. Journal of Machine Learning Research, 3(March):1157-1182, 2003. 3

55. I. Guyon, J. Weston, and S. Barnhill. Gene selection for cancer classification using support vector machine. Machine Learning, 46(1-3):389-422, 2002. 5

56. Mark A. Hall. Correlation-based Feature Selection for Machine Learning. PhD thesis, Department of Computer Science, University of Waikato, 1999. 4

57. Mark A. Hall and Lloyd A. Smith. Feature selection for machine learning: Comparing a correlation-based filter approach to the wrapper. In Proceedings of the 
Twelfth Florida International Artificial Intelligence Research Symposium Conference, pages 235-239, Menlo Park, CA, 1999. The AAAI Press. 4

58. Peter Hall, Byeong U. Park, and Richard J. Samworth. Choice of neighbor order in nearest-neighbor classification. The Annals of Statistics, 36(5):21352152, 2008. 30

59. David Hand, Heikki Mannila, and Padhraic Symyth. Principles of Data Mining. The MIT Press, Cambrige, MA, 2001. 2, 61

60. T. Hastie, R. Tibshirani, G. Sherlock, M. Eisen, P. Brown, and D. Botstein. Imputing missing data for gene expression arrays. Technical report, Stanford University Statistics Department, 1999. URL http://www-stat.stanford.edu/ ^hastie/Papers/missing.pdf. 6

61. Trevor Hastie and Robert Tibshirani. Discriminant adaptive nearest-neighbor classification. IEEE Transactions on Pattern Analysis and Machine Intelligence, 18:607-616, 1996. 7

62. Trevor Hastie, Robert Tibshirani, and Jerome Friedman. The Elements of Statistical Learning - Data Mining, Inference, and Prediction, chapter 9, section 2. Springer, New York, 2001. 5, 10

63. Piotr. Indyk and Rajeev Motwani. Approximate nearest neighbors: Towards removing the curse of dimensionality. In Proceedings of 30th Symposium on Theory of Computing, 1998. URL http://people.csail.mit.edu/indyk/nndraft.ps. 25

64. The Broad Institute. Cancer program data sets:. URL http://www.genome. wi.mit.edu/cgi-bin/cancer/datasets.cgi. 63 
65. George H. John, Ron Kohavi, and Karl Pfleger. Irrelevant features and the subset selection problem. In Machine Learning: Proceedings of the Eleventh International Conference on Machine Learning, pages 121-129, 1994. 4

66. Kenji Kira and Larry A. Rendell. A practical approach to feature selection. In ML92: Proceedings of the ninth international workshop on Machine learning, pages 249-256, San Francisco, CA, 1992. Morgan Kaufmann Publishers Inc. 4

67. S. Kirkpatrick, C.D. Gelatt, and M.P. Vecchi. Optimization by simulated annealing. Science, New Series, 220(4598):671-680, 1983. 5

68. Ron Kohavi and George H. John. Wrappers for feature selection. Artificial Intelligence, 97(1-2):273-324, 1997. 4

69. Igor Kononenko, Edvard Šimec, and Marko Robnik-Šikonja. Overcoming the myopia of inductive learning algorithms with relieff. Applied Intelligence, 7(1): 39-55, 1997. 4

70. R. Krauthgamer and J.R. Lee. Navigating nets: simple algorithms for proximity search. In SODA '04: Proceedings of the fiffteenth annual ACM-SIAM symposium onDiscrete algorithms, pages 798-807, Philadelphia, PA, 2004. Society for Industrial and Applied Mathematics. 25

71. Pat Langley. Selection of relevant features in machine learning. In Proceedings of the AAAI Fall Symposium on Relevance (1994), New Orleans, LA, 1994. AAAI Press. 3

72. Mark Last, Abraham Kandel, and Oded Maimon. Information-theoretic algorithm for feature selection. Pattern Recognition Letters, 22(6-7):799-811, 2001. 4 
73. Kent Leahy. Multicollinearity: When the solution is the problem. In Olivia Parr Rud, editor, Data Mining Cookbook. John Wiley \& Sons, Inc., New York, 2000. 61

74. J-H. Lee and C-J. Lin. Automatic model selection for support vector machines, 2000. URL http://www.csie.ntu.edu.tw/ ${ }^{\sim}$ cjlin/papers/modelselect.ps. gz. 5

75. Shengqiao Li, Adam Fedorowicz, Harshinder Singh, and Sidney C. Soderholm. Application of the random forest method in studies of local lymph node assay based skin sensitization data. Journal of Chemical Information and Modeling, 45(4):952-964, 2005. 11

76. Shenqqiao Li, Adam Fedorowicz, and Michael Andrew. A new descriptor selection scheme for SVM in unbalanced class problem: a case study using skin sensitisation dataset. SAR and QSAR in Environmental Research, 18(5-6):423441, 2007. 5

77. Yi Lin and Yongho Jeon. Random forests and adaptive nearest neighbors. Journal of the American Statistical Association, 101(474):578-590, 2006. 11

78. Huan Liu and Rudy Setiono. A probabilistic approach to feature selection: a filter solution. In Proceedings of the thirteenth International Conference on Machine Learning, pages 319-337, 1996. 4

79. D.B. Lomet and B. Salzberg. The hB-tree: A multiattribute indexing method with good guaranteed perfomance. In Proceedings of ACM TODS, volume 15, pages 625-658, December 1990. 25 
80. Nathan Mantel and Bernard S. Pasternack. A class of occupancy problems. The American Statistician, 22(2):23-24, 1968. 14

81. Barry H. Margolin and Jr. Winokur, Herbert S. Exact moments of the order statistics of the geometric distribution and their relation to inverse sampling and reliability of redundant systems. Journal of the American Statistical Association, 62(319):915-925, 1967. 18

82. P. Mitra, C.A. Murthy, and S.K. Pal. Unsupervised feature selection using feature similarity. Pattern Analysis and Machine Intelligence, IEEE Transactions on, 24(3):301-312, March 2002. 4

83. Michael Mitzenmacher and Eli Upfal. Probability and Computing: Randomized Algorithms and Probabilistic Analysis. Cambridge University Press, 2005. ISBN 0521835402, 9780521835404. 19

84. Robert M. Mnataskanov, N. Misra, Shengqiao Li, and E. James Harner. $k_{n^{-}}$ nearest neighbor estimators of entropy. Mathematical Methods of Statistics, 17 (3):261-277, 2008. 30

85. E.H. Moore. On the reciprocal of the general algebraic matrix. Bulletin of the American Mathematical Society, 26:394-395, 1920. 60

86. Songyot Nakariyakul and David P. Casasent. Adaptive branch and bound algorithm for selecting optimal features. Pattern Recognition Letters, 28(12):14151427, 2007. 5

87. NCI. Dtp - cell lines in the in vitro screen. URL http://dtp.nci.nih.gov/ docs/misc/common_files/cell_list.html. 63 
88. S.A. Nene and S.K. Nayar. A simple algorithm for nearest neighbor search in high dimensions. Pattern Analysis and Machine Intelligence, IEEE Transactions on, 19(9):989-1003, 1997. 25

89. C.M. Nunes, Jr. Ad.S. Britto, C.A.A. Kaestner, and R. Sabourin. An optimized hill climbing algorithm for feature subset selection: evaluation on handwritten character recognition. Ninth International Workshop on Frontiers in Handwriting Recognition (IWFHR-9), pages 365-370, October 2004. 5

90. Hanchuan Peng, Fuhui Long, and Chris Ding. Feature selection based on mutual information criteria of max-dependency, max-relevance, and min-redundancy. IEEE Transactions on Pattern Analysis and Machine Intelligence, 27(8):12261238, August 2005. 4

91. Roger Penrose. A generalized inverse for matrices. Proceedings of the Cambridge Philosophical Society, 51:406-413, 1955. 60

92. Franz Pernkopf and Paul O'Leary. Floating search algorithm for structure learning of bayesian network classifiers. Pattern Recognition Letters, 24(15):28392848, 2003. 5

93. P. Pudil, F.J. Ferri, J. Novovičová, and J. Kittler. Floating search methods for feature selection with nonmonotonic criterion functions. In Pattern Recognition, 1994. Vol. 2 - Conference B: Computer Vision \& Image Processing, Proceedings of the 12th IAPR International Conference on, volume 2, pages 279-283, October 1994. 58

94. P. Pudil, J. Novovičová, and J. Kittler. Floating search methods in feature selection. Pattern Recognition Letters, 15:1119-1125, 1994. 
95. P. Pudil, J. Novovičová, and P. Somol. Feature selection toolbox software package. Pattern Recognition Letters, 23(4):487-492, 2002. 5, 58

96. Sridhar Ramaswamy, Ken N. Ross, Eric S. Lander, and Todd R. Golub. A molecular signature of metastasis in primary solid tumors. Nature Genetics, 33 (1):49-54, 2003. 35

97. N. Roussopoulos and D. Leifker. Direct spatial search on pictorial database using packed r-trees. In Proceeding of ACM SIGMOD, May 1985. 25

98. G. Schwarz. Estimating the dimension of a model. The Annals of Statistics, 6 (2):461-4643, 1978. 4

99. Shayle R. Searle. Matrix Algebra Useful for Statistics, chapter 15, section 3. John Wiley \& Sons, New York, 1982. 61

100. T. Sellis, N. Roussopoulos, and C. Faloutsos. The r+-tree: A dynamic index for multitimensional objects. In Proceedings of 13th International Conference on VLDB, pages 507-519, September 1987. 25

101. Robert H. Shoemaker. Timeline: The NCI60 human tumour cell line anticancer drug screen. Nature Reviews Cancer, 6:813-823, 2006. 63

102. R. Short and K. Fukunaga. The optimal distance measure for nearest neighbor classification. IEEE Transaction on Information Theory, IT-27(5):622-627, 1981. 7

103. P. Somol, P. Pudil, J. Novovičová, and P. Paclík. Adaptive floating search methods in feature selection. Pattern Recognition Letters, 20(11-13):1157-1163, 1999. 5,58 
104. Guo-Jie Song, Shi-Wei Tang, Dong-Qing Yang, and Teng-Jiao Wang. A spatial feature selection mehtod based on maximum entropy theory. Journal of Software, 14(9):1544-1550, 2003. 4

105. D.A. Sprott. A note on a class of occupancy problems. The American Statistician, 23(2):12-13, 1969. 14

106. W. Stadje. The collector's problem with group drawings. Advances in Applied Probability, 22(4):866-882, 1990. 14

107. Jane E. Staunton, Donna K. Slonim, Hilary A. Coller, Pablo Tamayo, Michael J. Angelo, Johnny Park, Uwe Scherf, Jae K. Lee, William O. Reinhold, Weinstein John N., Jill P. Mesirov, Eric S. Lander, and Todd R. Golub. Chemosensitivity prediction by transcriptional profiling. Proceedings of the National Academy of Sciences, 98(19):10787-92, September 2001. 63

108. V. Svetnik, A. Liaw, C. Tong, J.C. Culberson, R.P. Sheridan, and B.P. Feuston. Random forest: A classification and regression tool for compound classification and qsar modeling. Journal of Chemical Information and Computer Science, 43 (6):1947-1958, 2003. 10

109. R Development Core Team. R: A Language and Environment for Statistical Computing. R Foundation for Statistical Computing, Vienna, Austria. URL http://www.r-project.org. ISBN 3-900051-07-0. 37

110. Sergios Theodoridis and Konstantinos Koutroumbas. Pattern recognition. Academic Press, 2003. 3

111. Robert Tibshirani. Regression shrinkage and selection via the lasso. Journal of the Royal Statistical Society. Series B (Methodological), 58(1):267-288, 1996. 70 
112. Olga Troyanskaya, Michael Cantor, Gavin Sherlock, Pat Brown, Trevor Hastie, Robert Tibshirani, David Botstein, and Russ B. Altman. Missing value estimation methods for DNA microarrays. Bioinformatics, 17(6):520-525, 2001. 6

113. J.K. Uhlmann. Metric trees. Applied Mathematics Letters, 4(5):61-62, 1991. 25

114. J.K. Uhlmann. Satisfying general proximity/similarity queries with metric trees. Information Processing Letters, 40(4):175-179, 1991. 25

115. Laura J. van 't Veer, Hongyue Dai, Marc J. van de Vijver, Yudong D. He, Augustinus A.M. Hart, Mao Mao, Hans L. Peterse, Karin van der Kooy, Matthew J. Marton, Anke T. Witteveen, Ron M. Kerkhoven George J. Schreiber and, Chris Roberts, Peter S. Linsley, René Bernards, and Stephen H. Friend. Gene expression profiling predicts clinical outcome of breast cancer. Nature, 415:530-536, 2002. 36

116. J. Weston, S. Mukherjee, O. Chapelle, M. Pontil, T. Poggio, and V. Vapnik. Feature selection for svms. In Advance in Neural Information Processing Systems, volume 13, pages 668-674, Cambridge, MA, 2001. MIT Press. 5

117. Colin White. The committee problem. The American Statistician, 25(4):25-26, 1971. 14

118. D.C. Whitley, M.G. Ford, and D.J. Livingstone. Unsupervised forward selection: A method for eliminating redundant variables. Journal of Chemical Information and Computer Science, 40(5):1160-1168, 2000. 4

119. H. Wolfson. Model-based object recognition by geometric hashing. In Proceed- 
ings of the First European COnferencr on Computer Vision, pages 526-536, April 1990. 25

120. Eric P. Xing, Michael I. Jordan, and Richard M. Karp. Feature selection for high-dimensional genomic microarray data. In ICML '01: Proceedings of the Eighteenth International Conference on Machine Learning, pages 601-608, San Francisco, CA, USA, 2001. Morgan Kaufmann Publishers Inc. 4

121. Howard Hua Yang and John Moody. Feature selection based on joint mutual information. In Proceedings of International ICSC Symposium on Advances in Intelligent Data Analysis, pages 22-25, 1999. 4

122. Sheng Yang and Jun Gu. Feature selection based on mutual information and redundancy-synergy coefficient. Journal of Zhejiang University SCIENCE, 5 (11):1382-1391, 2004. 4

123. Lei $\mathrm{Yu}$ and Huan Liu. Feature selection for high-dimensional data: A fast correlation-based filter solution. In Proceedings of the Twentieth International Conference on Machine Learning (ICML-2003), 2003. 4

124. Lei Yu and Huan Liu. Redundancy based feature selection for microarray data. In KDD'04: Proceedings of the tenth ACM SIGKDD international conference on Knowledge discovery and data mining, pages 737-742, New York, NY, 2004. ACM.

125. Lei $\mathrm{Yu}$ and Huan Liu. Efficient feature selection via analysis of relevance and redundancy. The Journal of Machine Learning Research, 5:1205-1224, 2004. 4

126. Hong Lin Zhai, Xing Guo Chen, and Zhi De Hu. A new approach for the 
identification of important variables. Chemometrics and Intelligent Laboratory Systems, 80(1):130-135, January 2006. 5 